\title{
THz streaking at metal nanotips
}

\section{DISSERTATION}

zur Erlangung des mathematisch-naturwissenschaftlichen Doktorgrades

\author{
„Doctor rerum naturalium” \\ der Georg-August-Universität Göttingen \\ im Promotionsprogramm ProPhys \\ der Georg-August University School of Science (GAUSS) \\ von \\ Lara Simone Wimmer \\ aus Göttingen
}

Göttingen, 2018 
Betreuungsausschuss

Prof. Dr. Claus Ropers, 4. Physikalisches Institut

Prof. Dr. Tim Salditt, Institut für Röntgenphysik

Mitglieder der Prüfungskommission

Referent: $\quad$ Prof. Dr. Claus Ropers, 4. Physikalisches Institut

Korreferent: $\quad$ Prof. Dr. Stefan Mathias, 1. Physikalisches Institut

Weitere Mitglieder der Prüfungskommission

Prof. Dr. Ansgar Reiners, Institut für Astrophysik

Prof. Dr. Tim Salditt, Institut für Röntgenphysik

Prof. Dr. Andreas Tilgner, Institut für Geophysik

Prof. Dr. Cynthia A. Volkert, Institut für Materialphysik

Tag der mündlichen Prüfung: 30. Januar 2018 


\section{Abstract}

This thesis addresses various aspects of terahertz $(\mathrm{THz})$ streaking at metallic nanotips. In $\mathrm{THz}$ near-field streaking, electron pulses emitted by femtosecond near-infrared (NIR) pulses are accelerated in a THz-induced near-field at the tip apex. The energy of the electrons is measured in streaking spectrograms as a function of the relative time delay between the $\mathrm{THz}$ and the NIR pulses. The spatial confinement of the enhanced $\mathrm{THz}$ near-field facilitates characteristic electron dynamics, in which the electrons escape the near-field within a small fraction of an optical half-cycle.

In the first part of this thesis, the influence of a static voltage onto the electron dynamics is investigated. The measurements show that a bias voltage applied to the tip acts as an additional control parameter of the spectrograms. For a spatiotemporal characterization of the near-field, the streaking spectrograms are simulated numerically using parameters such as the field strength at the tip surface and the spatial field decay length. In addition, the simulations enable the analysis of the electron trajectories and the study of the temporal evolution of the electron pulse in phase space demonstrating a control of the phase space volume.

In a second study, THz streaking at plasmonic nanotapers is employed to determine the propagation velocity of plasmons. The experimental results are in agreement with simulations of the plasmon velocity as a function of the taper diameter.

The possibilities of $\mathrm{THz}$ streaking at metal nanotips for the control and manipulation of electron pulses can be used for example in time-resolved electron microscopy and diffraction.

\section{Zusammenfassung}

Thema dieser Arbeit sind verschiedene Aspekte von terahertz (THz) Streaking an metallischen Nanospitzen. In THz Nahfeld-Streaking werden von Femtosekunden-Pulsen im Infrarotbereich emittierte Elektronenpulse in einem THz-induzierten Nahfeld am Apex der Spitze beschleunigt. Die Energie der Elektronen wird in Streaking Spektrogrammen als Funktion des zeitlichen Abstands zwischen den THz- und den Nahinfrarotpulsen gemessen. Bedingt durch die geringe Ausdehnung des stark überhöhten Nahfeldes zeigt sich dabei eine spezielle Elektronendynamik, bei der die Elektronen das THz Nahfeld in einem geringen Bruchteil eines optischen Halbzyklus' verlassen.

Im ersten Teil dieser Arbeit wird der Einfluss einer statischen Vorspannung auf die Elektronendynamik untersucht. Die experimentellen Ergebnisse zeigen, dass eine an der Spitze angelegte Vorspannung wie ein zusätzlicher Kontrollparameter auf die Spektrogramme wirkt. Die Streaking Spektrogramme werden numerisch simuliert für eine räumliche und zeitliche Charakterisierung des Nahfeldes durch Parameter wie die Feldstärke an 
der Metalloberfläche und die räumliche Abklinglänge des Feldes. Zudem ermöglichen die Simulationen eine Untersuchung der Elektronentrajektorien und der zeitlichen Entwicklung des Elektronenpulses im Phasenraum. Dabei erlaubt die räumliche und zeitliche Inhomogenität des $\mathrm{THz}$ Nahfeldes eine Kontrolle des Phasenraumvolumens.

In einer zweiten Studie wird THz Streaking an plasmonischen Nanospitzen dazu verwendet, die Propagationsgeschwindigkeit von Plasmonen zu bestimmen. Die Messergebnisse stimmen gut überein mit den Resultaten einer Simulation der Propagationsgeschwindigkeit als Funktion des Spitzendurchmessers.

Die Möglichkeiten zur Kontrolle und Manipulation von Elektronenpulsen, die THz Streaking an metallischen Nanospitzen bietet, können für zeitaufgelöste Experimente zum Beispiel in der Elektronenmikroskopie und -Beugung verwendet werden. 


\section{Contents}

1 Introduction $\quad 1$

2 Field enhancement and photoemission at metal nanostructures $\quad \mathbf{5}$

2.1 Field enhancement and localization . . . . . . . . . . . . . 5

2.2 Photoemission processes . . . . . . . . . . . . . . . . . . . . . . 9

2.3 Adiabaticity parameter . . . . . . . . . . . . . . . 12

3 Fundamentals of $\mathrm{THz}$ streaking at metal nanotips $\quad \mathbf{1 5}$

3.1 All-optical streaking . . . . . . . . . . . . . . . . . . . 15

3.2 Near-field sampling and electron pulse control by $\mathrm{THz}$

streaking at metal nanotips . . . . . . . . . . . . . . . 17

$3.3 \mathrm{THz}$ tunneling in $\mathrm{THz}$ streaking spectrograms . . . . . . . . . . . . . . 20

4 Plasmonic excitation of metallic nanostructures $\quad \mathbf{2 5}$

4.1 Dielectric function of metal . . . . . . . . . . . . . . . . 25

4.2 Propagating surface plasmon polaritons . . . . . . . . . . . . 26

4.3 Plasmon excitation on planar surfaces . . . . . . . . . . . . . . 28

4.4 Adiabatic nanofocusing . . . . . . . . . . . . . . . . . . 30

5 Experimental methods 33

5.1 Experimental setup . . . . . . . . . . . . . . . . . . 33

5.2 Generation of the $\mathrm{THz}$ transients using the $\mathrm{AC}$ bias method . . . . . . . . 35

5.3 Time-of-flight spectrometer . . . . . . . . . . . . . . 36

5.4 Data processing . . . . . . . . . . . . . . . . . . 38

5.5 Electro-optic sampling . . . . . . . . . . . . . . . . . . . 39

6 Phase space manipulation of free-electron pulses from metal nanotips $\begin{array}{ll}\text { using combined terahertz near fields and external biasing } & 43\end{array}$

6.1 Introduction . . . . . . . . . . . . . . . . . . . 43

6.2 Experimental procedure and results . . . . . . . . . . . . 46

6.3 Numerical simulation of streaking spectrograms . . . . . . . . . . . . . . 48

6.4 Influence of the near-field parameters on the electron dynamics . . . . . . 49

6.5 Caustic trajectories . . . . . . . . . . . . . . . . . 5 50

6.6 Evolution in phase space . . . . . . . . . . . . . . . 52

6.7 Conclusions . . . . . . . . . . . . . . . . . . . . 53

6.8 Appendix A: Experimental methods and data analysis . . . . . . . . . . 53

6.9 Appendix B: Numerical simulations and analytical streaking model . . . . 54

6.10 Appendix C: Computation of the phase space density distribution . . . . 61

7 Clocking plasmon nanofocusing by THz near-field streaking $\quad 63$ 
8 Discussion $\quad 69$

8.1 Summary: Aspects of $\mathrm{THz}$ streaking at metal nanotapers . . . . . . . . . 69

8.2 Application of $\mathrm{THz}$ streaking in time-resolved electron microscopy . . . . 71

8.3 Experimental aspects . . . . . . . . . . . . . . . . . . . . 74

8.4 Future simulations . . . . . . . . . . . . . . . . . . . 80

8.5 Conclusions . . . . . . . . . . . . . . . . . . . 81

$\begin{array}{lc}\text { A Abbreviations and symbols } & 83\end{array}$

$\begin{array}{lr}\text { B Simulations } & \mathbf{8 7}\end{array}$

B.1 Simulation of the emission process . . . . . . . . . . . . 87

B.2 Simulation of the electron trajectories . . . . . . . . . . . . 90

B.3 Simulation of the phase space density distribution . . . . . . . . . . 91

B.4 Simulation of the tunneling spectra . . . . . . . . . . . . . . . 93

$\begin{array}{ll}\text { Bibliography } & 101\end{array}$

$\begin{array}{ll}\text { Author contributions, publications and conference talks } & 117\end{array}$

$\begin{array}{lr}\text { Curriculum Vitae } & 121\end{array}$

$\begin{array}{lr}\text { Danksagung } & 123\end{array}$ 


\section{Chapter 1}

\section{Introduction}

The interaction of light and matter is one of the most fundamental topics in physics. Interactions of light with bound and mobile electrons determine the material-specific refraction and absorption properties. Under specific circumstances, light can induce electron emission from metal surfaces. The kinetic energy of the emitted electrons is provided by the photon energy in the photoelectric effect [1]. For high light intensities, the electron energy depends on the field strength of the incident electromagnetic wave. In this strong-field regime, the electron emission is due to an optically-induced tunneling process. Such high field strengths can be reached in the enhanced near-field of metallic nanostructures. The specific effects occurring for particles with a size in the range of the incident wavelength are described in nano-optics.

This thesis addresses the influence of light in the near-infrared (NIR) and terahertz $(\mathrm{THz})$ spectral range on metallic nanostructures. In particular, the photoelectron dynamics in a THz-induced near-field of a gold nanotip are investigated. This topic is embedded in the wider context of manipulating electron pulses for time-resolved electron probe techniques. For example in ultrafast tunneling microscopy, THz radiation can be used for the visualization of molecular processes on the femtosecond time and nanometer length scale as demonstrated, e.g., by T. L. Cocker in Ref. [2].

In general, the observation of processes on the femtosecond and attosecond scale requires measurement mechanisms on the relevant time scales, which can be essentially found in free electron bunches and in electromagnetic pulses in the x-ray, ultraviolet (UV) and visible range. In optics, elaborated methods for the generation and control of bandwidth-limited pulses are applied to optimize the temporal resolution in optical microscopy, diffraction and spectroscopy. Due to the short wavelength of electrons, the spatial resolution of electron microscopy is superior to optical techniques. In principle, bandwidth-limited electron pulses would be shorter than optical pulses. However, the temporal reshaping of electron pulses still remains challenging.

The importance of the pulse duration for the measurements of ultrafast processes can 
be illustrated by the example of the pump-probe scheme. This widely distributed technique is based on two temporally delayed pulses. First, a pump pulse excites the sample, and afterwards, the probe pulse measures the relaxation of the system. The temporal resolution of pump-probe experiments is directly determined by the pulse durations of both pulses. Thus, the generation of ultrashort electromagnetic and electron pulses is the primary aim for an improvement of the time resolution. In the following, the most widely used methods for the temporal and spatial manipulation of electromagnetic and electron pulses are briefly introduced.

By now, the shortest measured optical pulse durations are in the attosecond range (Review on attosecond physics in Ref. [3]). For the measurement of the pulse duration, a special class of pump-probe experiments was developed, which is all-optical streaking. In attosecond streaking, attosecond extreme ultraviolet (XUV) and NIR pulses are used. The XUV pulses generate photoelectrons in an atomic gas. The subsequent acceleration of these electrons in the electric field of the NIR pulse depends on their time of emission, i.e., the temporal structure of the emission process is projected on the energy axis of the electrons. The kinetic energy spectra as a function of the delay between the two pulses, called "streaking spectrogram", follow the vector potential of the NIR pulse and thus, allow for a phase-resolved measurement of a light wave [4, 5]. All-optical streaking enables on the one hand a measurement of the attosecond pulse duration, and on the other hand, the XUV pulse serves as a probe pulse to track the vector potential of the near-infrared pulse. The concept of streaking was also transferred to lower frequencies down to the $\mathrm{THz}$ range $[6,7]$.

The spatial resolution of optical microscopy is confined by the size of the light spot, which is given by the diffraction limit due to the wave characteristics of light. Methods to increase the optical resolution down to the nanometer region are based on the superposition of two foci [8] or on the measurement of single photons [9]. For these techniques, the Nobel Prize in chemistry 2014 was awarded to R. E. Betzig, S. W. Hell, and W. E. Moerner. An alternative method for the generation of a light spot with a diameter below the diffraction limit is near-field microscopy [10], which uses the feature of nanostructures to locally enhance incident electric fields. The size of this so-called "near-field" is determined by the radius of curvature of the nanostructure and can be far below the diffraction limit.

The shortest, just recently measured electron pulse durations are also in the sub-femtosecond range. The experiments implemented by K. Priebe et al. [11] and by Y. Morimoto and P. Baum [12] utilize a quantum-coherent interaction of free electron pulses with a tailored light field. Alternatively, electron pulses can be compressed in optical cavities, imprinting an energy distribution on an electron pulse that leads to a minimal pulse duration at the sample. In this method, the electron pulses are temporally compressed at the cost of an increased energy spread and typically, the phase space volume of the electron pulse is preserved [13]. This method, which is closely related to streaking, was also realized in the $\mathrm{THz}$ frequency range by C. Kealhofer et al. in Ref. [14]. 
A special version of optical streaking is investigated in this thesis, using the spatially confined near-field of a metal nanotip for the acceleration of electron pulses [15]. In this case, the near-field is induced by $\mathrm{THz}$ pulses, which facilitates characteristic electron dynamics enabled by the spatiotemporal inhomogeneity of the near-field. These electron dynamics, discussed in detail by G. Herink et al. in Ref. [16], allow for the electrons to escape the near-field in a small fraction of an optical half-cycle, such that the kinetic energy of the electrons is proportional to the electric field in the moment of emission. Thus, the waveform of the near-field can be measured directly.

For increasing electron propagation times in the $\mathrm{THz}$ near-field, propagation effects appear in the spectrograms, i.e., the electron energy gain in the near-field also depends on the temporal gradient of the field and on the initial energy of the photoelectrons. Hence, the energy distribution of the electron pulses can be manipulated. Due to these propagation effects, streaking at metal nanostructures offers significantly more possibilities for the manipulation of electron pulses than conventional streaking, including the control of the space volume.

The possibilities of $\mathrm{THz}$ streaking for the spatiotemporal measurement of the near-field and the control of electron pulses are investigated in detail in this thesis. In addition, the application of the streaking concept for the study of plasmon propagation on a nanotip is introduced. The experimental results are complemented by simulations facilitating access to the electron trajectories and to the phase space density distribution.

\section{Outline}

This cumulative thesis comprises two publications:

1. Phase space manipulation of free-electron pulses from metal nanotips using combined terahertz near fields and external biasing

L. Wimmer, O. Karnbach, G. Herink, and C. Ropers.

Physical Review B 95165416 (2017)

\section{Clocking plasmon nanofocusing by THz near-field streaking}

L. Wimmer, B. Schröder, M. Sivis, G. Herink, and C. Ropers.

Appl. Phys. Lett. 111, 131102 (2017)

These articles are presented in the Chapters 6 and 7. They are accompanied by an introduction into the topics building the foundation of the present work. In Chapter 2, the theoretical basics are explained, which are the various mechanisms of field enhancement at nanostructures, different kinds of emission mechanisms and a classification parameter for electron dynamics in a spatiotemporally varying electric field. Chapter 3 summarizes our previous work on $\mathrm{THz}$ streaking at metal nanotips published in Refs. [15, 17]. The 
principles of plasmonics are briefly reviewed in Chapter 4. A description of the experimental methods is given in Chapter 5 .

The results of this thesis are discussed in Chapter 8, giving an outlook to the application of $\mathrm{THz}$ streaking for the reshaping of electron pulses in electron microscopy and to further future projects based on the concept of $\mathrm{THz}$ streaking.

Chapter 6 shows simulations of the streaking spectrograms, the electron trajectories and the phase space density of the electron pulses. These simulations are explained in detail in the Appendix B. 


\section{Chapter 2}

\section{Field enhancement and photoemission at metal nanostructures}

In $\mathrm{THz}$ streaking at metal nanotapers, the characteristic features of the spectrograms arise from the nanometric spatial confinement and the strong enhancement of the NIR field and the THz field at the tip apex. The enhanced near-field at the apex allows for localized photoemission. However, the photoemission processes in the NIR and the $\mathrm{THz}$ ranges are different.

In this Chapter, the essential theoretical concepts that explain the experimental results of $\mathrm{THz}$ streaking are introduced. First, the origin of the field enhancement in the surrounding of nanostructures is explained. A second Section addresses different emission processes and their characterization by the Keldysh parameter. In the third and last Section of this Chapter, the classification of electron dynamics in a spatiotemporally varying electric field by a spatial adiabaticity parameter is discussed. In THz streaking, the high spatiotemporal inhomogeneity of the THz-induced near-field at the tip apex gives rise to characteristic photoelectron trajectories governed by the electric field in the moment of photoemission.

\subsection{Field enhancement and localization}

Nanoparticles locally enhance incident electric fields due to a redistribution of surface charges. The distribution of electrons close to the surface compensates for the external field, shielding the bulk metal from its surrounding. These electrons generate an electric field directly above the metal surface that enhances the incident field. The strength of that field depends on the characteristics of the external field and on the shape of the nanostructure. For static electric fields, the local enhancement at structures with a small 


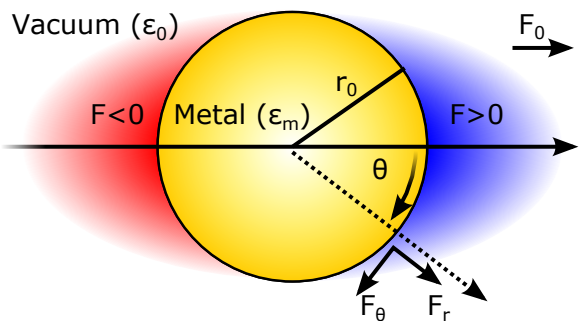

Figure 2.1: A spherical nanoparticle in a homogeneous electric field. The red and the blue shades to both sides of the particle indicate the dipole field.

radius of curvature is due to the "lightning rod effect", named after its most prominent application invented in the $18^{\text {th }}$ century by B. Franklin.

For metal nanoparticles with a size in the range of the incident wavelength, antenna effects come into play. Nanostructures react in a similar way to light waves as macroscopic radio antennas respond to radio waves with a wavelength in the millimeter to kilometer range. The excitation efficiency of these plasmonic nanoantennas depends on the antenna resonances.

The lightning rod effect as a response to static electric fields and the antenna effects induced by electromagnetic waves lead to a strong local enhancement of incident fields, as discussed in the following. Both effects play a role in $\mathrm{THz}$ streaking at metal nanotapers.

\subsubsection{Static field enhancement}

The static field enhancement in the vicinity of metal nanostructures can be deduced from the boundary conditions for the electric field at a metal-vacuum interface. Here, the electric field exhibits a continuous transition for the field $F^{\|}$parallel to the surface and a discrete step for the field $F^{\perp}$ perpendicular to the surface. Mathematically, these boundary conditions are described in agreement with the Maxwell's equations by (see, e.g., Ref. [18, p. 379])

$$
\begin{aligned}
\lim _{|\vec{x}| \nearrow r_{0}} \vec{F}^{\|} & =\lim _{|\vec{x}| \searrow r_{0}} \vec{F}^{\|}=0, \\
\varepsilon_{m} \cdot \lim _{|\vec{x}| \nearrow r_{0}} \vec{F}^{\perp} & =\varepsilon_{0} \cdot \lim _{|\vec{x}| \searrow r_{0}} \vec{F}^{\perp} .
\end{aligned}
$$

In this equation, $r_{0}$ represents the radius of a spherical particle (see Fig. 2.1). However, the boundary conditions are valid for arbitrarily shaped metal surfaces. The permittivities for the two half-spaces are $\varepsilon_{0}$ in vacuum and $\varepsilon_{m}$ in metal.

For an isolated, uncharged metal nanoparticle in a homogeneous electric field, the field is given by a purely dipolar distribution $[19$, p. 15]:

$$
\begin{gathered}
F_{r}=F_{0} \cos \theta+2 F_{0} \frac{r_{0}^{3}}{r^{3}} \cos \theta, \\
F_{\theta}=F_{0} \sin \theta-F_{0} \frac{r_{0}^{3}}{r^{3}} \sin \theta .
\end{gathered}
$$


The electric field $\vec{F}$ is given in radial coordinates with $r$ being the distance to the center of the particle that is the origin of the coordinate system and $\theta$ being the angle measured from an axis parallel to the homogeneous field (cf. Fig. 2.1).

The boundary conditions correspond to an electric field pointing perpendicularly to the metal surface. This implies a high local field enhancement for structures with a small radius of curvature, e.g., the tip of a lightning rod or the apex of a nanotaper.

In contrast to a spherical nanoparticle, it is not possible to compute the electric field of a generic biased nanotaper analytically. A first estimation of the enhanced surface electric field $F_{0}$ is given by [20, p. 41]:

$$
F_{0}=\frac{U_{\text {bias }}}{k_{f} r_{0}}
$$

with $U_{\text {bias }}$ as the bias voltage, $r_{0}$ as the apex radius of curvature, and $k_{f}$ as the field factor, which is expected to be in the range of 3-8 [20-22], depending on the opening angle of the tip.

Employing several approximations concerning, e.g., the opening angle and the tip to sample distance $d_{z}$, analytical expressions for the electric field along the tip axis of a hyperbolical taper can be obtained [20, p. 47]:

$$
F\left(d_{z}\right)=\frac{F_{0}}{1+\frac{2}{r_{0}} d_{z}} .
$$

This field distribution shows exemplary that the electrical field of a biased nanotip decays on longer distances than the field of an equally charged sphere, which exhibits the decay of a monopole.

In the experimental setup employed in this thesis, the distance between the biased nanotip and the grounded entrance of the time-of-flight spectrometer amounts to $3 \mathrm{~mm}$, and it can be assumed that the lightning rod effect does not influence the electric field close to the detector. Thus, we describe the electric field of the nanotips in our experiment as a superposition of a dipolar component with the surface electric field $F_{\text {stat }}^{0}$ and a homogeneous electric field $F_{\text {stat }}^{1}$ :

$$
F(r)=F_{\text {stat }}^{0} \frac{d^{3}}{(d+r)^{3}}+F_{\text {stat }}^{1} .
$$

In this equation, $d$ is the decay length of the dipolar component.

\subsubsection{Optical antenna excitation}

The complex dielectric function of a metal in combination with a nanostructure geometry determines the optical response of a nanotip to incident light. For infrared and visible frequencies, nanostructures are acting as optical antennas that exhibit resonance conditions for most efficient excitation similar to macroscopic antennas. In contrast to 
radio antennas, the penetration depth of the optical driving field into the metal, i.e., the skin depth, and the inertia of the electrons in the antenna have to be taken into account. As for radio antennas, resonances can be derived from the concept of standing waves for antenna lengths $l$ of multiples $n$ of the half wavelength: $l=n \lambda_{e f f} / 2$. The effective wavelength $\lambda_{\text {eff }}$ is the wavelength "seen" by the antenna, defined as [23]:

$$
\lambda_{e f f}=n_{1}+n_{2} \frac{\lambda}{\lambda_{p}}
$$

Here, $\lambda$ is the vacuum wavelength, $\lambda_{p}$ the plasma wavelength of the metal, and $n_{1}$ and $n_{2}$ are parameters depending on the geometry and the dielectric characteristics of the metal. For often used geometries and materials such as gold or silver, $\lambda_{\text {eff }}$ is typically 2-6 times shorter than the incident wavelength.

The relation between the incident frequency and the resonance frequency determines the excitation efficiency and the phase lag between the incident wave and the near-field of the antenna.

Metal nanotips can be seen as a semi-infinite antenna, and the fact that the optical focus covers only a part of the structure complicates the description of the near-field. In general, a higher field enhancement can be expected for longer wavelengths due to larger oscillation amplitudes of the surface charges in the metal. In the THz range, the near-field is expected to be mainly in-phase with the incident waveform, indicating that the excitation wavelength is larger than the resonance wavelength.

Generally, the antenna response of a nanotip can be described within the framework of an RLC circuit, with the dissipated power $P_{\text {diss }}$ in relation to the incident power $P_{\text {inc }}$ given by [24, 25]:

$$
\frac{P_{\text {diss }}}{P_{\text {inc }}}=\frac{R Z_{0}}{4 \pi\left(R^{2}+\left(\omega L-\frac{1}{\omega C}\right)^{2}\right)} .
$$

In this equation, $Z_{0}$ is the vacuum impedance, $R$ the resistance, $\omega$ the angular frequency, $L$ the inductance and $C$ the capacity. This model characterizes the changes between the incident waveform and the near-field.

In our experiments, a strong enhancement of the incident field but only minor deviations between the incident waveform and the near-field in the main optical cycle are observed. After the main optical cycle, oscillations occur in the streaking measurements that do not appear in the electro-optic sampling trace. In addition to the complex antenna response, also the spatial distribution of the $\mathrm{THz}$ frequencies within the focus [26-28] contributes to deviations between the spatially-averaged measurement of the incident electric field and the streaking trace. 

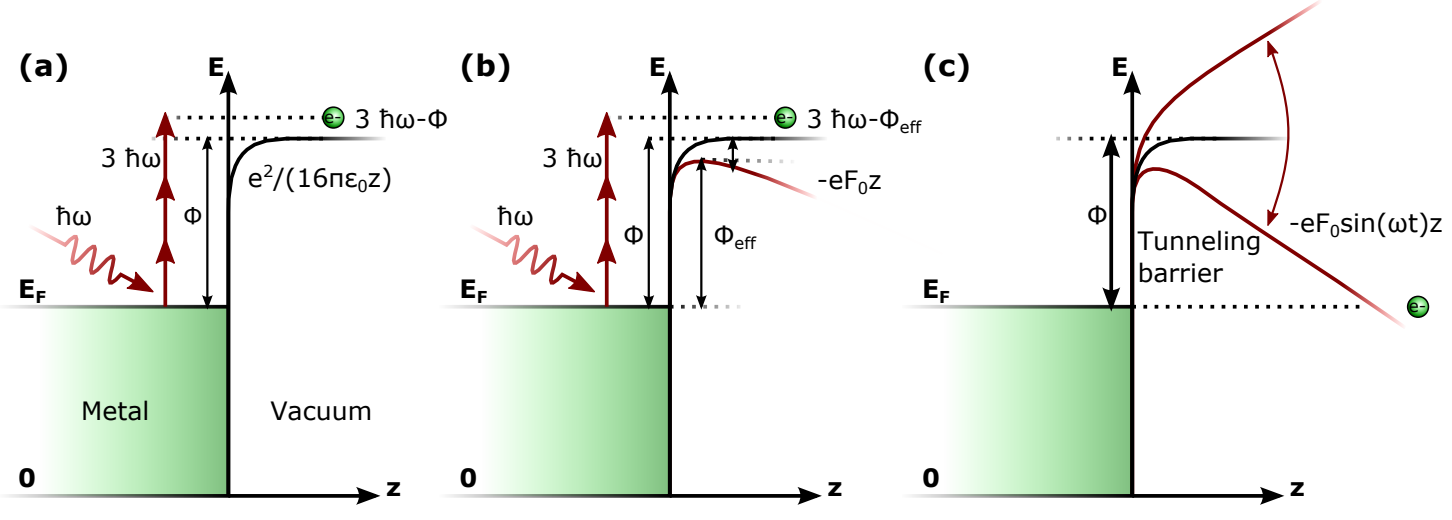

Figure 2.2: Potential energy distribution in the vicinity of a metal surface. (a) Multiphoton photoemission in a field-free surrounding. The potential energy distribution in vacuum close to the surface is influenced by image charges. (b) A homogeneous external electric field bends the potential energy distribution down. In combination with the image charge effect, this leads to a decrease of the effective work function $\phi_{e f f}$. (c) Optically-induced tunneling in the electric field of an electromagnetic wave.

\subsection{Photoemission processes}

Two qualitatively different photoemission processes occur in our implementation of $\mathrm{THz}$ streaking at metal nanotips: NIR-induced multiphoton photoemission and THz-induced tunneling. Both pulses influence the photoemission caused by the respective other pulse. The THz field affects the multiphoton photoemission due to the Schottky effect altering the metal work function. The excitation of a hot carrier distribution in the metal by the NIR pulse changes the number of tunneling electrons.

In this Section, first the Schottky effect is described, followed by an introduction to the Keldysh parameter to characterize the photoemission processes that are discussed subsequently.

\subsubsection{Schottky effect}

The Schottky effect describes the reduction of a metal work function caused by an electric field that bends down the potential barrier at the metal-vacuum interface. The bending of the potential barrier in combination with the image charge effect leads to a reduction of the barrier height. This effect was first discovered by W. Schottky in 1923 [29].

If an electron is brought close to a metal surface, charge carriers in the metal are rearranged due to image charges, generating a potential energy distribution $E_{\text {pot }}(z)$ in a distance $z$ from the metal-vacuum interface ([30, p. 10], see Fig. 2.2(a)):

$$
E_{\text {pot }}(z)=\frac{e^{2}}{16 \pi \varepsilon_{0} z}
$$


This equation is deduced from the Coulomb force between a test charge in distance $z$ from the surface and its image charge in the metal. If this potential energy distribution is superimposed with the potential energy of a homogeneous electric field $F_{0}$, the height of the potential barrier is changed by the energy $\Delta E$ ([30, p. 10], see Fig. 2.2(b)):

$$
\begin{array}{r}
E_{\text {pot }}(x)=-\frac{e^{2}}{16 \pi \varepsilon_{0} z}+e F_{0} z \text { and } \\
\Delta E=\sqrt{\frac{e^{3} F_{0}}{4 \pi \varepsilon_{0}}} .
\end{array}
$$

The reduction of the work function $\Phi$ to an effective work function $\Phi_{\text {eff }}=\Phi-\Delta E$ (cf. Fig. 2.2(b)) leads to an enhancement of the photocurrent, and a strong bending of the potential barrier facilitates tunneling of electrons from the metal into the vacuum. The Schottky effect is included in the simulations discussed in detail in Appendix B.

\subsubsection{Keldysh parameter}

Photoemission processes can be classified by the ratio between the work function and the ponderomotive energy $U_{p}$, defined as (see, e.g., Ref. [31, p. 573])

$$
U_{p}=\frac{e^{2} F^{2}}{4 m_{e} \omega^{2}},
$$

with the elementary charge $e$ and the mass of the electron $m_{e}$. The ponderomotive energy is the average kinetic energy of an electron oscillating in a quiver motion in the field of an electromagnetic wave.

The Keldysh parameter $\gamma$ relates the work function $\Phi$ to the ponderomotive energy [32]:

$$
\gamma=\sqrt{\frac{\Phi}{2 U_{p}}} .
$$

If the ponderomotive energy is negligible compared to the work function $(\gamma \gg 1)$, the photoemission can be described in terms of photons by multiphoton photoemission, i.e., the electron overcomes the work function using the energy of one or more photons (cf. Fig. 2.2(a)).

A small ponderomotive energy can be taken into account in the photon picture. In order to emit an electron, the photons have to provide enough energy for the quiver motion of the electron in addition to the work function:

$$
\Phi_{e f f}=\Phi+U_{p}
$$

If the contribution of the ponderomotive energy is large enough, the minimum number of photons required to overcome the effective work function $\Phi_{\text {eff }}$ increases, changing the photon order of the emission process. This effect is known as "channel closing" [33]. 
For small Keldysh parameters $\gamma \ll 1$, the ponderomotive energy is much larger than the work function, and the emission process can be described in the strong-field picture. Here, the photoemission can be treated as an optically-induced tunneling process (cf. Fig. 2.2(c)).

For short wavelengths in the optical range, the tunneling regime cannot be reached easily at metal nanotips, since the local intensities required for a ponderomotive energy exceeding the work function may destroy the structure.

For intensities used in our experiments, the Keldysh parameter indicates multiphoton photoemission in the NIR range and a THz-driven tunneling process. If two pulses with different Keldysh parameters temporally overlap, mixed processes can appear, e.g., the absorption of a photon with a subsequent tunneling process.

\subsubsection{Multiphoton photoemission}

In the multiphoton regime, several photons are simultaneously absorbed to emit one electron (cf. Fig. 2.2(a)). The number of the emitted electrons scales with the incident intensity and with the photon order $n$ of the emission process:

$$
J_{n} \propto I^{n}
$$

The photocurrent density $J_{n}$ is described by the Fowler-DuBridge model [34-37] for a Fermi-distributed free electron gas:

$$
J_{n}=a_{n} A_{0}\left(\frac{e}{\hbar \omega}\right)^{n}(1-\varrho)^{n} I^{n} T^{2} \mathcal{F}\left(\frac{n \hbar \omega-e \Phi_{e f f}}{k_{b} T}\right)
$$

Here, $a_{n}$ is a material-dependent coefficient, $\varrho$ the reflectivity, $I$ is the intensity, $A \approx$ $120 \frac{\mathrm{A}}{\mathrm{m}^{2} \mathrm{~K}^{2}}$ the Richardson coefficient, $k_{b}$ the Boltzmann constant, and $T$ the temperature of the Fermi-distributed free-electron gas. The Fowler function $\mathcal{F}$ is defined as [34]:

$$
\mathcal{F}(x)=\int_{0}^{\infty} \ln \left(1+e^{x-y}\right) d y
$$

A detailed derivation of these equations can be found, e.g., in the work of $R$. B. Brogle [38]. The Fowler-DuBridge model is employed to simulate an energy spectrum of multiphoton-emitted electrons as discussed in Appendix B. 
(a)

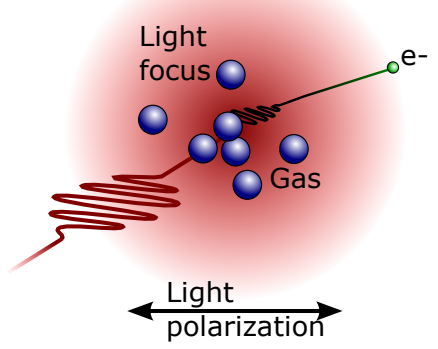

(b)

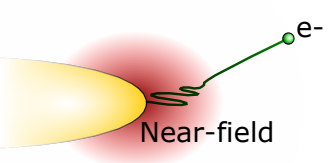

(c)

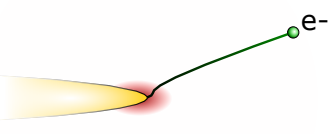

Figure 2.3: Electron dynamics in spatially a inhomogeneous optical driving field. (a) Photoemission from a gas in a diffraction-limited focus. (b) The photoelectron leaves the spatially confined near-field of a nanotip after multiple oscillations. (c) The electron escapes in less than one optical half-cycle (Figure adapted from Ref. [40]).

\subsubsection{Tunneling photoemission}

In the strong-field regime, the photocurrent does not depend on the photon energy but on the field amplitude of the electromagnetic wave, and the photoemission appears predominantly in phases of high negative field strength. Optically-induced tunneling emission (cf. Fig. 2.2(c)) can be treated as a Fowler-Nordheim tunneling process [39] with the current density $J$ in its most simple form:

$$
J(F) \propto \Theta(-F) F^{2} \exp \left(-\frac{4}{3} \sqrt{\frac{2 m_{e}}{\hbar^{2}}} \frac{\Phi^{3 / 2}}{e F}\right) .
$$

Here, $\Theta$ is the Heaviside function. This expression was originally developed to describe a tunneling process caused by a static electric field, but can be also used to approximate optical tunneling (see Appendix B).

\subsection{Adiabaticity parameter}

In $\mathrm{THz}$ streaking, the electrons are accelerated after their emission in the THz-induced near-field at the tip apex. The acceleration of electrons in a spatially inhomogeneous electric field can be characterized by a spatial adiabaticity parameter $\delta$ that relates the quiver amplitude $l_{q}$ of the electron in the field of an electromagnetic wave to the spatial decay length $l_{f}$ of the field [16]:

$$
\delta=\frac{l_{f}}{l_{q}}
$$

where the quiver amplitude is given by

$$
l_{q}=\frac{e F}{m_{e} \omega^{2}} .
$$


If the spatial adiabaticity parameter is large compared to unity $(\delta \gg 1)$, the spatial inhomogeneity of the electric field is negligible and does not influence the electron trajectories that are purely governed by the quiver motion in the optical driving field. This is the case in diffraction-limited foci for short wavelengths and moderate intensities as illustrated in Fig. 2.3(a). The final momentum $p$ of the electrons is proportional to the vector potential $A$ of the pulse in the moment of emission $t_{0}[41]$ :

$$
p=p_{0}-e \int_{t_{0}}^{\infty} F(t) d t=e A\left(t_{0}\right) .
$$

The initial electron momentum $p_{0}$ is gained from the photoemission process.

In the case of long pulses and moderately localized electric fields, the electron oscillates multiple cycles in the optical driving field before leaving the spatially confined field as depicted in Fig. 2.3(b). The electron experiences the spatial decay of the electric field and obtains an additional energy of $1 U_{p}$ in comparison to the propagation in a spatially homogeneous field [42].

The high localization and enhancement of the near-field at metallic nanostructures allows for adiabaticity parameters below one $(\delta<1)$ for large wavelengths. If the spatial decay length is smaller than the quiver amplitude, the electron escapes the electric field in less than one optical half-cycle (Fig. 2.3(c)). Here, the electron energy is strongly influenced by the spatial decay of the electric field, and the electron trajectories become more complex [43]. Depending on the initial kinetic energy after the emission, the electron trajectories can significantly differ from each other.

For quiver amplitudes much larger than the spatial decay length of the electric field $(\delta \ll 1)$, the electron energy is proportional to the electric field in the moment of photoemission. This regime of field-driven electron dynamics is only reached in the near-field of nanostructures for long wavelengths and in phases of high field amplitudes [15].

The adiabaticity parameter does not take into account the impact of an additional static electric field. An analogous way to describe electron acceleration in the spatially inhomogeneous electric field of an optical pulse is in terms of escape times [42, 44, 45]. In this thesis, the spatial adiabaticity parameter is used as an estimate of the electron dynamics. The influence of the initial electron energy and a static bias on the electron trajectories are studied in detail in Chap. 6. 



\section{Chapter 3}

\section{Fundamentals of $\mathrm{THz}$ streaking at metal nanotips}

This Chapter introduces $\mathrm{THz}$ near-field streaking and THz-induced tunneling at metal nanotips. First, the concept of all-optical streaking is briefly reviewed in the context of attosecond physics, and specific aspects of streaking in the near-field of nanostructures are elucidated.

Second, our previous studies on $\mathrm{THz}$ streaking $[15,17]$ are summarized with an emphasis on the experimental results. The setup used for the measurements presented in this thesis is described in detail in our publications $[15,17]$ and in Chap. 5. The simulations complementing the measurements are discussed in Appendix B.

Streaking in the near-field of a nanostructure was first reported in our work "Terahertz control of nanotip photoemission" [15], presenting a reconstruction of the full near-field waveform and a spectral reshaping of electron pulses in the near-field.

Upon increasing the THz field strength, THz-induced tunneling emission is observed. The tunneling current depends on the relative time delay between the NIR and the $\mathrm{THz}$ pulse and can be applied to track the relaxation of a NIR-induced hot carrier distribution. This study was published as "Field emission at terahertz frequencies: AC-tunneling and ultrafast carrier dynamics" by G. Herink et al. [17].

\subsection{All-optical streaking}

Originally, the term "streaking" applies to a method for time-resolved measurements, based on the time-dependent acceleration of photoelectrons $[46,47]$. In the first streaking experiments, the electron beam was electronically deflected in a direction orthogonal to the propagation direction by a temporally increasing electric field. The point of arrival at the detector is directly correlated to the emission time of the electron, i.e., the time-axis is projected onto a spatial axis. This type of streak camera usually exhibits a 


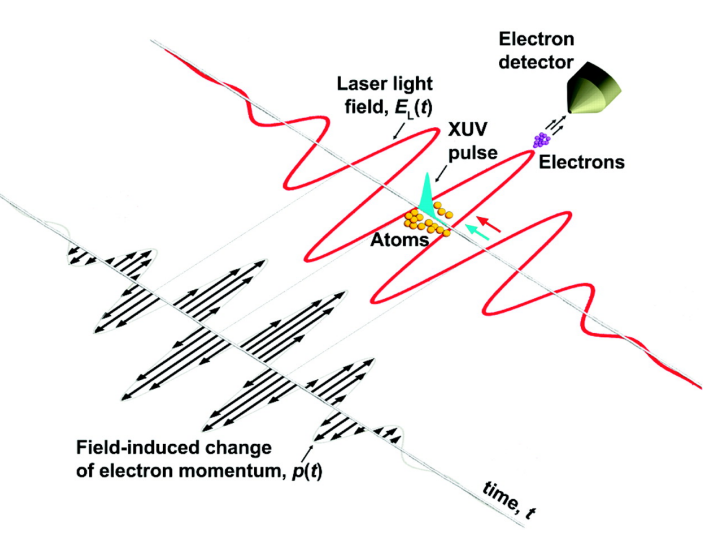

(a) Experimental scheme.

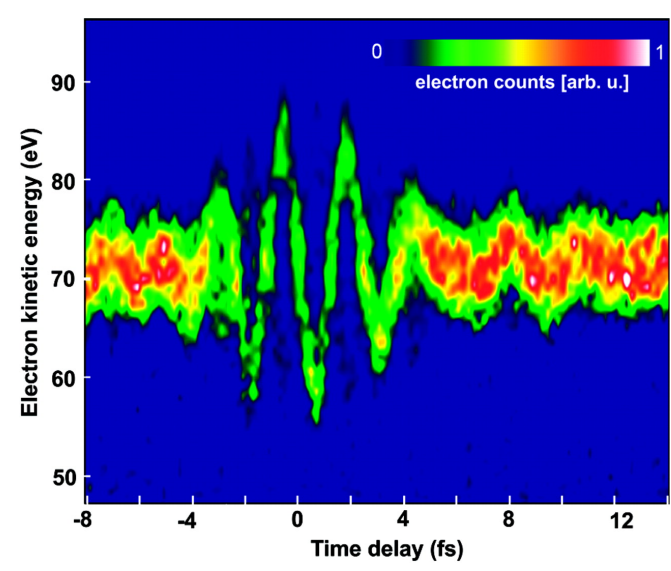

(b) Measured streaking spectrogram.

Figure 3.1: Attosecond streaking (From [5]. Reprinted with permission from AAAS.).

time resolution in the picosecond range, limited by the temporal gradient of the streaking field.

The time-resolution can be drastically improved by using optical pulses for the acceleration of the photoelectrons, provided that the half-period of the accelerating pulse is long compared to the electron pulse duration. Optical half-cycles of increasing electric field accelerate the electrons depending on their emission time such that the temporal structure of the electron pulse is mapped on the velocity of the particles.

All-optical streaking was first introduced in attosecond science [4, 5, 41, 48, 49]. In attosecond streaking, photoelectrons are emitted from gas atoms by XUV pulses and are accelerated in the electric field of phase-stable NIR pulses (cf. Fig. 3.1a, Reviews $[3,50])$. The kinetic energy spectra of the photoelectrons are recorded as a function of the relative pulse delay (see Fig. 3.1b). These measurements, referred to as "streaking spectrograms", are used to determine the XUV pulse duration and the waveform of the accelerating pulse. The concept of all-optical streaking was extended from attosecond XUV pulses to a broad range of frequencies including the THz spectrum [6, 7]. The $\mathrm{THz}$ streaking experiments are performed for the single-shot characterization of x-ray [6] and electron pulses [7].

Typically, these streaking experiments are performed in gaseous media in diffraction limited foci and thus, the momentum distribution follows the vector potential of the optical driving field corresponding to the temporal integral of the electric field (see Eqn. 2.22 or, e.g., [41]). In the case of sinusoidal field transients, the carrier-envelope phase of the vector potential is shifted by $\pi / 2$ compared to the electric field. The dynamics become more complex in the vicinity of nanostructures due to the spatial inhomogeneity of the near-field (cf. Sec. 2.3 and Refs. [42, 44, 51-53]).

For the experimental implementation of near-field streaking, the choice of the nanostructure and the wavelength of the optical driving pulse are of major importance, determining the electron dynamics as described in Sec. 2.3. A small radius of the surface curvature allows for a high field enhancement and a strong localization of the near-field, 
both facilitating sub-cycle dynamics. A long wavelength and a high incident intensity increase the quiver amplitude, promoting electron propagation driven by the electric field in the moment of photoemission. In addition to the expected electron dynamics, the damage threshold of the material has to be considered, which makes low frequencies more suitable for near-field streaking in the sub-cycle regime.

\subsection{Near-field sampling and electron pulse control by $\mathrm{THz}$ streaking at metal nanotips}

We implemented near-field streaking employing $\mathrm{THz}$ transients and single metal nanotips [15]. Using a single nanostructure with only one emission site allows for the study of this specific individual near-field.

With incident field strengths up to $100 \mathrm{kV} / \mathrm{cm}$, the $\mathrm{THz}$ transients used in our experiment enable electron dynamics deeply in the sub-cycle regime without damaging the tip. In such field-driven spectrograms, the streaking trace follows the THz electric field in the moment of emission (cf. Sec. 3.2.1). Upon increasing the field decay length or decreasing the THz field strength, propagation effects occur, facilitating a spectral reshaping of the electron pulses (cf. Sec. 3.2.2).

\subsubsection{Near-field reconstruction}

For sharp nanotips, field-driven electron dynamics as discussed in Sec. 2.3 can be reached. The Figures 3.2(b) and (d) show exemplary streaking spectrograms for a nanotip with a radius of curvature of $10 \mathrm{~nm}$.

Due to the asymmetric geometry of the experiment, photoemission only appears for a negative field strength at the tip surface. Thus, a single streaking spectrogram recorded with low bias voltage shows only the accelerating half-cycles of the $\mathrm{THz}$ near-field. To resolve the complete waveform, access to the hidden half-cycles is required, where the $\mathrm{THz}$ field is positive and drives electrons back into the metal surface. These half-cycles can be accessed by either drawing the electrons to the detector by a sufficiently high tip bias (cf. Chap. 6) or reversing the sign of the THz field, as it is done here. The $\mathrm{THz}$ pulses are generated with the AC bias method (see Sec. 5.2), where the carrier-envelope phase of the $\mathrm{THz}$ transient is tunable. The Figures 3.2(b) and (d) show two streaking spectrograms using $\mathrm{THz}$ transients of opposite polarity. As depicted in Fig. 3.2(c), the two spectrograms complement each other to a combined waveform that represents the $\mathrm{THz}$ near-field. Figure 3.2(a) displays the incident $\mathrm{THz}$ transients for both polarities for comparison. The combined streaking trace matches to the incident waveform except for a slight phase-shift and the small oscillations following the main single-cycle pulse. These oscillations are due to the antenna excitation of the tip by the $\mathrm{THz}$ pulse (cf. Sec. 2.1.2). 


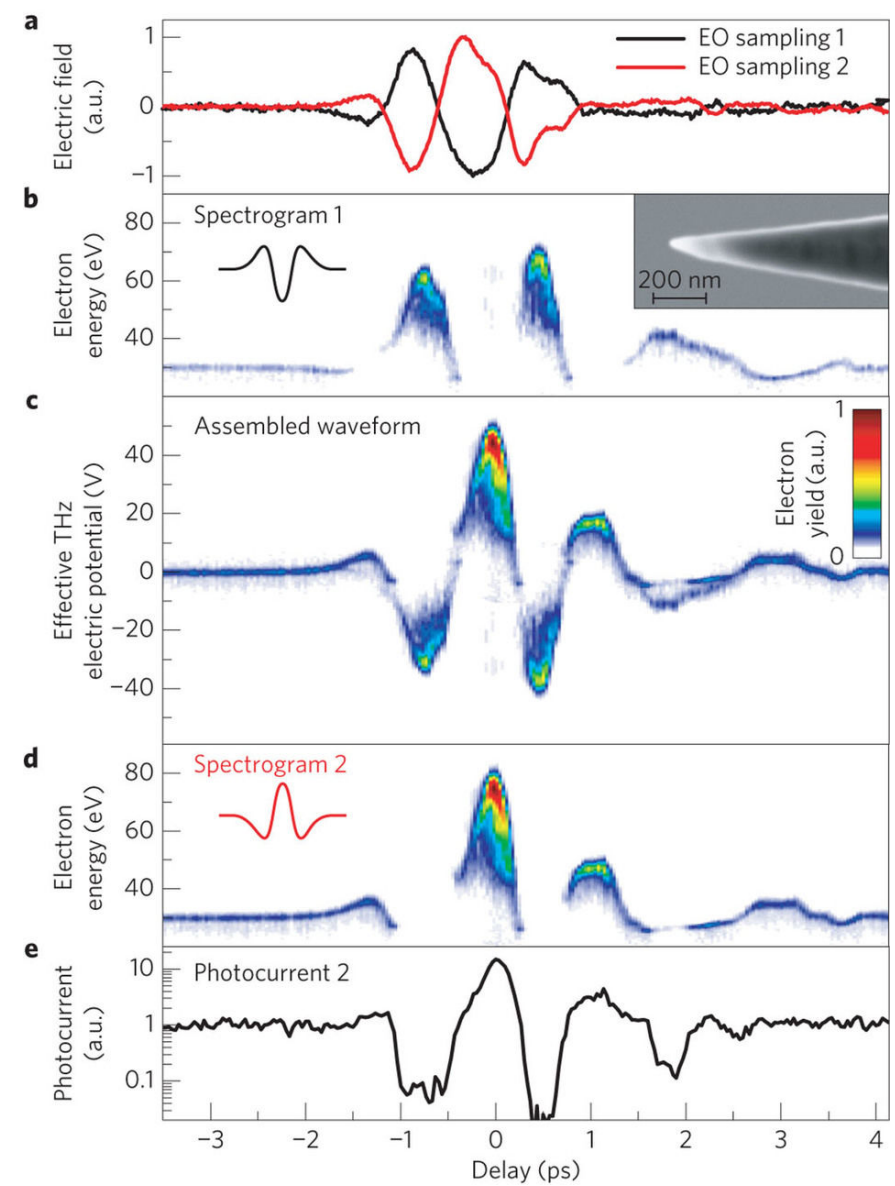

Figure 3.2: Reconstruction of the full near-field waveform using two complementary streaking spectrograms recorded at sharp nanotip (inset in (b), apex radius $10 \mathrm{~nm}$ ). (a) Electro-optic sampling measurements in $\mathrm{ZnTe}$ at the tip position for two opposite field polarities. (b) and (d) streaking measurements corresponding to the waveforms marked in black and red. (c) A combination of the two spectrograms shows the complete near-field waveform. (e) THz-induced photocurrent modulation of the spectrogram shown in (d) (Figure taken from Ref. [15], (C)npg).

In $\mathrm{THz}$ streaking, the moment of emission within the $\mathrm{THz}$ optical cycle is controlled by the relative pulse delay. Only for emission times directly at the zero-crossings of the surface electric field (see, e.g., Fig. 3.2(d) at -0.4 ps), the electron experiences propagation effects due to the low field-strength in the moment of emission also for large $\mathrm{THz}$ field amplitudes. In all other phases, the streaking trace maps the $\mathrm{THz}$ near-field in the moment of photoemission.

Figure 3.2(e) shows strong THz-induced modulations of the photocurrent that is deduced from the streaking spectrogram in Fig. 3.2(d). The photocurrent enhancement in phases of large negative field strength is due to the Schottky effect (cf. Sec. 2.2.1). The nearly complete suppression of the current at -0.8 ps and 0.5 ps is caused by an inhibition of the photoemission at a positive surface field and the acceleration of already emitted electrons back to the tip.

\subsubsection{Reshaping of electron energy spectra}

For nanotips with a larger radius of curvature $r_{0}>20 \mathrm{~nm}$, the $\mathrm{THz}$ near-field decays on larger length scales, and the field enhancement is lower than for the sharp nanotips discussed above. Both effects lead to an increase of the propagation time in the electric 

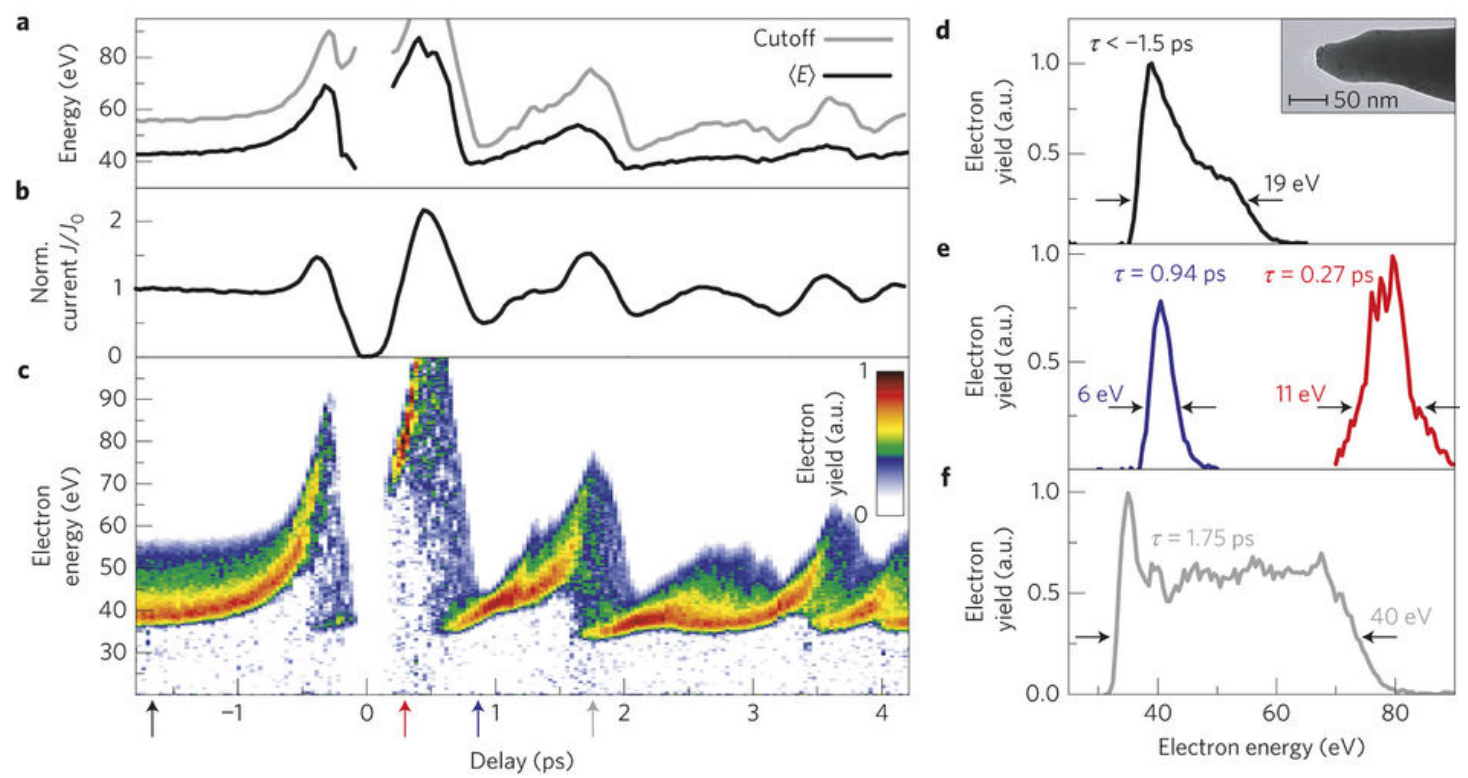

Figure 3.3: Spectral reshaping in $\mathrm{THz}$ streaking caused by propagation effects. (a) Expectation value (black) and cutoff-energy (grey). (b) Normalized photocurrent. (c) Spectrogram. (d) Energy spectrum without influence of the THz pulse. Inset: SEM micrograph of the nanotip. (e) and (f) THz-induced spectral compression and broadening (Figure taken from Ref. [15], (Cnpg).

field and thus to propagation effects, i.e., the temporal evolution of the THz field influences the final electron kinetic energy not only at the zero-crossing but in all emission phases. An example for such a spectrogram is shown in Fig. 3.3(c). Here, the width of the energy spectra is governed by the time-derivative of the $\mathrm{THz}$ near-field. The propagation time of the electrons in the electric field depends on their initial energy directly after the photoemission. The electrons with the initially highest energy are the first to escape the near-field. The initially slower electrons have a longer integration time in the electric field. Thus, for temporally decreasing field strength, the initial energy spectra are compressed (see Fig. 3.4, left trajectories). This is shown experimentally, e.g., in Fig 3.3(e) in comparison to Fig. 3.3(d). For temporally increasing field strength, the initially fastest electrons are accelerated to higher energies than the slower ones which results in a spectral broadening as shown in Fig. 3.3(f).

The dependence of the spectral width on the time derivative of the THz transient leads to a phase-shift between the energy expectation value that is influenced by propagation effects and the high energy cutoff that still follows the momentary electric field.

The reshaping of the electron energy spectra is further analyzed in Chap. 6 and bears the potential for future applications in time-resolved electron microscopy and diffraction experiments. 


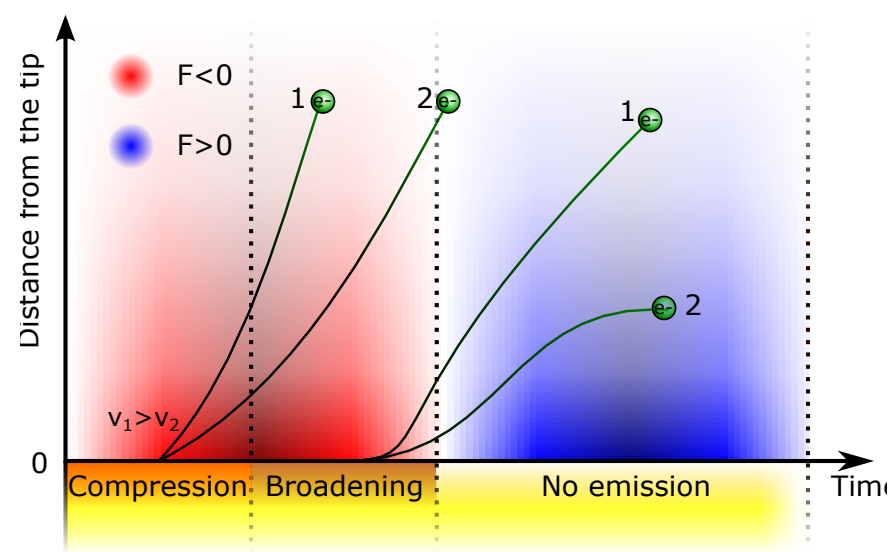

Figure 3.4: Trajectories illustrating the spectral compression and broadening, which depends on the time derivative of the $\mathrm{THz}$ electric field in the moment of emission.
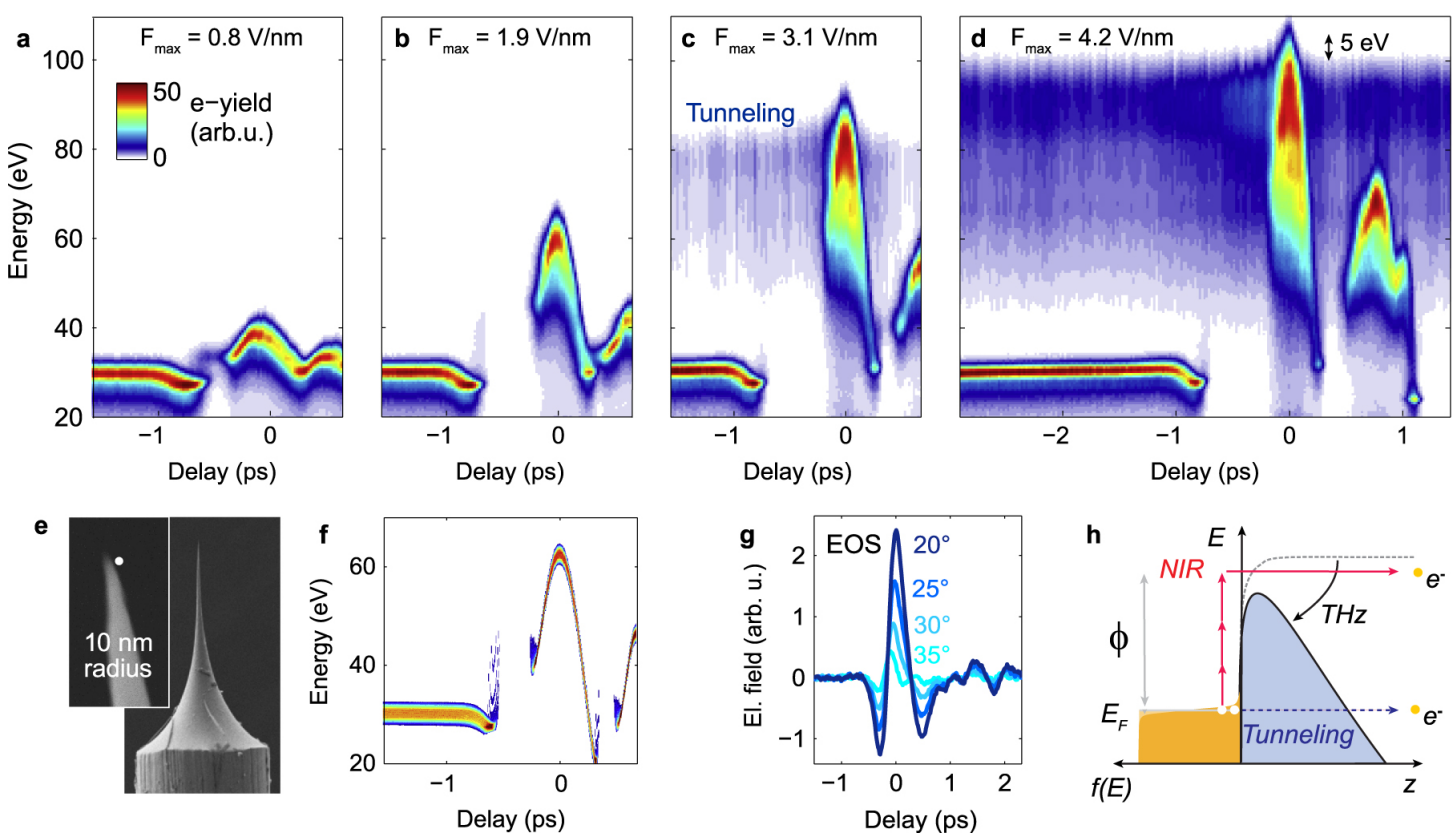

Figure 3.5: $\mathrm{THz}$ tunneling appears upon increase of the $\mathrm{THz}$ field strength in the spectrograms. (a)-(d) Streaking spectrograms for increasing field strength. (e) SEM micrograph of the tip used in the experiment. (f) Simulation of the streaking spectrogram without tunneling component. (g) Electro-optic sampling measurements of the incident $\mathrm{THz}$ transients in (a)-(d). (h) Sketch of the tunneling mechanism (Figure taken from Ref. [17]).

\subsection{THz tunneling in THz streaking spectrograms}

For higher incident THz field strengths and a small tip radius, a stripe of higher energetic electrons appears in the spectrogram as depicted in Fig. 3.5. This feature cannot be explained by the streaking scheme of NIR-induced photoemission and THz-driven acceleration since its kinetic energy is delay-independent. Upon further increasing the THz field strength (see Fig. 3.5(a)-(d)), the feature becomes more pronounced and the photoemission also occurs without NIR excitation of the tip (Fig. 3.6). The Keldysh parameter indicates that the $\mathrm{THz}$-induced emission is a tunneling process. 


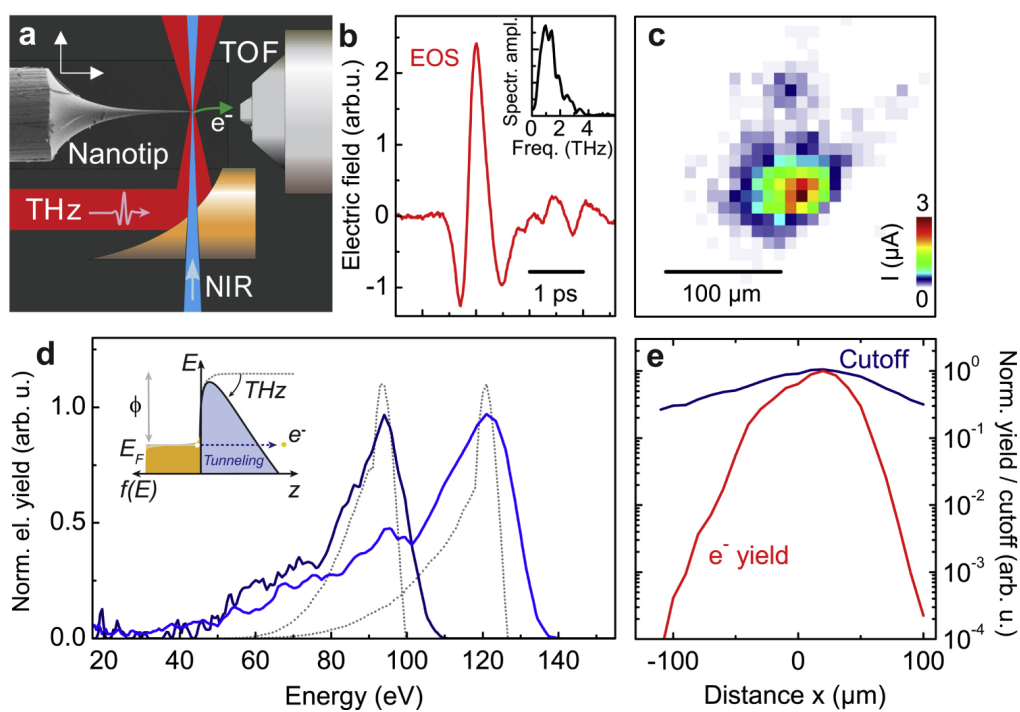

Figure 3.6: THz-induced tunneling. (a) Sketch of the experimental setup. (b) Electrooptic sampling trace of the incident $\mathrm{THz}$ transient. (c) Focus scan: tunneling current as a function of the tip position. (d) Two exemplary energy spectra for different local field strength $(3.1 \mathrm{~V} / \mathrm{nm}, 5.3 \mathrm{~V} / \mathrm{nm},-40 \mathrm{~V}$ bias). (e) Cutoff-energy in comparison to electron yield for a line-scan through the focus (Figure taken from Ref. [17]).

In the spectrogram in Fig. 3.5(d), there is a small energy difference between the maximum energy of the streaking trace and the maximum of the THz-induced spectra. This difference can be attributed to the work function of the metal-vacuum interface at the tip: The multiphoton emitted electrons have to overcome the potential barrier, while the THz-emitted electrons tunnel through it.

We analyzed the THz-induced tunneling emission in our work "Field emission at terahertz frequencies: AC-tunneling and ultrafast carrier dynamics" by G. Herink et al. [17] which is summarized in the following.

\subsubsection{THz-induced tunneling}

Figure 3.6 shows the THz-induced photoemission without an additional NIR excitation of the tip. The photocurrent depends on the position of the nanotip in the $\mathrm{THz}$ focus. A photocurrent map recorded by scanning the tip through the $\mathrm{THz}$ focus is depicted in Fig. 3.6(c). Due to the nonlinearity of the tunneling process, the diameter of the photocurrent spot is substantially smaller than the actual $\mathrm{THz}$ focus.

The kinetic energy spectra of the tunneling electrons exhibit a steep rise of the electron yield with increasing energy and are governed by a large peak at the maximum of the kinetic energy (cf. Fig. 3.6(d)). This high cutoff energy is proportional to the THz field strength.

The shape of the THz tunneling spectra in Fig. 3.6(d) can be explained by field-driven electron dynamics with a negligible integration time in the electric field and the strong nonlinearity of the emission process. The vast majority of the electrons is emitted in 
a

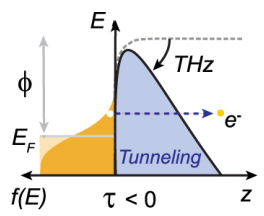

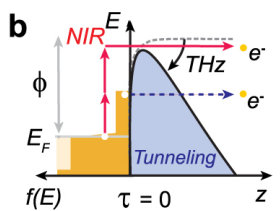

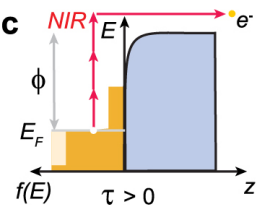

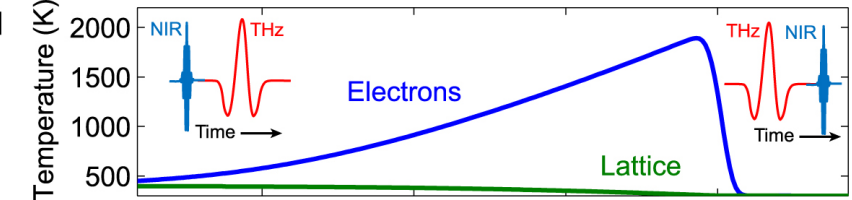

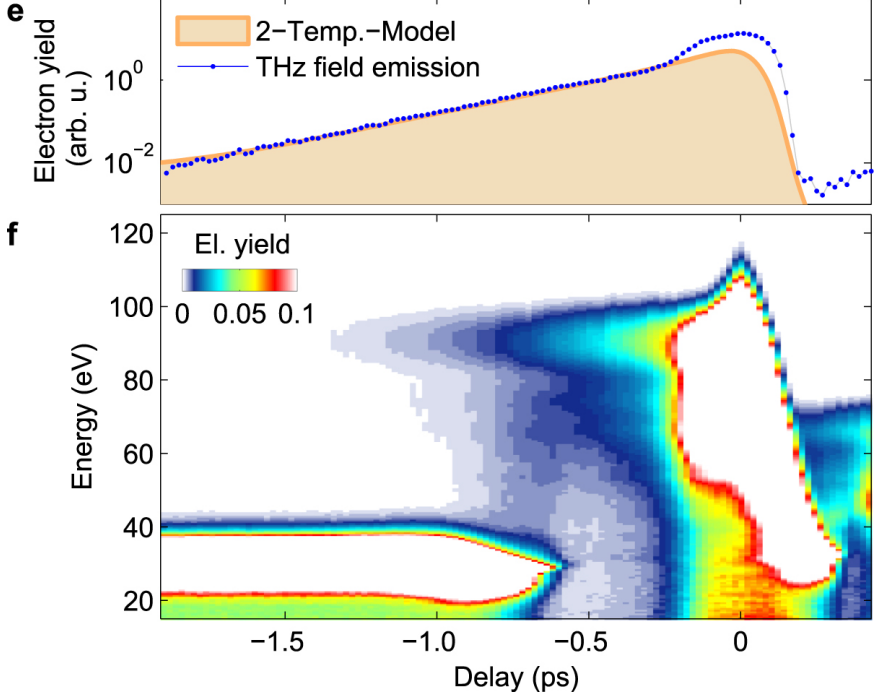

Figure 3.7: Probing the NIR-induced increase of the electron temperature employing the two temperature model. (a)-(c) Sketches of the emission processes. (d) Numerical computation of the electron and lattice temperature. (e) Simulated tunneling current in comparison to the measurement. ( $f$ ) Streaking measurement (Figure taken from Ref. [17]).

phases of a strong negative field strength. These electrons escape the near-field directly after the photoemission, thus, their kinetic energy is proportional to the maximimum of the THz field strength. In Figure 3.6(e), the high energy cutoff is compared to the photocurrent for a line scan through the focus. While the photocurrent increases nonlinearly with the surface electric field, the maximum kinetic energy scales linearly with the $\mathrm{THz}$ field for electron dynamics in the sub-cycle regime. Hence, the cut-off energy can be used as a measure for the diameter of the focus.

\subsubsection{Probing NIR-induced electron excitation}

In combination with the NIR beam, $\mathrm{THz}$ tunneling emission appears enhanced in the streaking spectrogram (see Fig. 3.7) at negative delays, where the NIR pulse arrives first at the tip and is followed by the THz pulse. Thus, the THz pulse acts on a NIR-induced hot electron distribution at the tip apex. With increasing electron temperature, more electrons are found at higher energies, at which the tunneling distance to the vacuum is smaller and, thus, the tunneling probability is higher (cf. Fig. 3.7). With increasing negative delay, the electron temperature is lowered at the arrival of the $\mathrm{THz}$ pulse, and the tunneling current declines. 
This decrease of the tunneling current can be used to track the relaxation of the NIRinduced excitation of the electrons. In this situation, the roles of pump and probe pulses are exchanged in comparison to the streaking trace. In THz streaking, the NIR-induced photoemission probes the $\mathrm{THz}$ field, while here, the tunneling current samples the NIRexcited electron distribution.

The increase of the electron temperature can be described by a two-temperature model (see Ref. [54] and Appendix B), the electron temperature determines the Fermi-distribution of the electrons in the metal and the photoemission is simulated as Fowler-Nordheim tunneling (cf. Sec. 2.2.4). These three steps directly connect the measured photocurrent to the momentary electron temperature.

As shown in Figure 3.7(b) and (c), the simulations are in good agreement with the measured photocurrent and exhibit a maximum electron temperature of more than $1500 \mathrm{~K}$. The numerical computations are described in more detail in Appendix B. 



\section{Chapter 4}

\section{Plasmonic excitation of metallic nanostructures}

In this Chapter, some aspects of plasmonics are briefly reviewed as an introduction to our experimental study presented in Chap. 7, in which $\mathrm{THz}$ streaking is applied to a plasmonic nanotaper. After a short summary of the dielectric properties of metals that enable plasmonic electron density waves, the characteristic properties of surface plasmons and the most commonly used experimental methods for the optical excitation of the plasmons are discussed, including grating coupling as implemented in our setup. In Chapter 7, plasmon propagation is studied on a metal nanotip that allows for adiabatic nanofocusing. Finally, the concept of adiabatic nanofocusing facilitating nanometric electron sources and a retardation of the plasmon propagation is introduced.

A comprehensive introduction into the field of plasmonics can be found in "Plasmonics Fundamentals and Applications" by S. Maier [55]. For surface plasmons in nano-optics, see, "Principles of nano-optics" by L. Novotny and B. Hecht [18].

\subsection{Dielectric function of metal}

In a first approximation, the dielectric function of a metal can be described by the Drude model that incorporates the conduction band electrons as a free electron gas. The binding potential of the metal ion cores is taken into account by an effective electron mass $m_{\text {eff }}$. The electron gas can be forced into an oscillating motion by incident electromagnetic waves. This electron motion in an electric field of frequency $\omega$ corresponds to a driven harmonic oscillator with the damping constant $\gamma_{d}=1 / \tau_{e}$, where $\tau_{e}$ is the relaxation time of the free electron gas, typically in the range of $10^{-14} \mathrm{~s}[55, \mathrm{p} .11]$. The electron motion causes a macroscopic polarization $\vec{P}$ and a displacement, which is in a 
linear approximation $\vec{D}=\varepsilon_{0} \vec{F}+\vec{P}=\varepsilon_{0} \epsilon \vec{F}$ with the dielectric permittivity $\varepsilon$ [55, p. 12]:

$$
\varepsilon(\omega)=1-\frac{\omega_{p}^{2}}{\omega^{2}+i \gamma_{d} \omega}
$$

The plasma frequency $\omega_{p}^{2}=n e^{2} / \varepsilon_{0} m_{\text {eff }}$ depends on the material and can be determined by electron energy loss spectroscopy (EELS). The plasma frequency of gold is $2.1 \mathrm{PHz}$ $(8.55 \mathrm{eV},[56])$, which corresponds to a wavelength of $145 \mathrm{~nm}$.

The dispersion relation of a traveling wave in a free electron gas is given by [55, p. 15]:

$$
\omega^{2}=\omega_{p}^{2}+k^{2} c_{0}^{2}
$$

Here, $c_{0}$ is the speed of light in vacuum and $k=2 \pi / \lambda$ is the wave vector. For frequencies below the plasma frequency $\omega<\omega_{p}$, the propagation of transverse electromagnetic waves is suppressed in the bulk, and the penetration depth of the electric field into the metal is described by the skin depth.

Frequencies above the plasma frequency allow for transverse waves with a group velocity $v_{g}=\mathrm{d} \omega / \mathrm{d} k<c_{0}$, i.e., the material becomes transparent for electromagnetic waves. With increasing frequencies, the dielectric function approaches unity. Noble metals exhibit strong d-bands closely below the Fermi level (for the band structure of gold, see, e.g., Ref. [57]) that cause a strong residual polarization for high excitation frequencies $\omega>\omega_{p}$ and the model has to be corrected by a dielectric constant at infinite frequency $\varepsilon_{\infty}$ to [55, p. 13]:

$$
\varepsilon(\omega)=\varepsilon_{\infty}-\frac{\omega_{p}^{2}}{\omega^{2}+i \gamma_{d} \omega}
$$

In the long wavelength limit $(k=0)$, the dispersion relation allows for longitudinal waves, oscillating at the plasma frequency $\omega_{p}$. These longitudinal oscillations, which exist at various frequencies, are called volume plasmons and do not couple to (transverse) electromagnetic waves. The excitation of volume plasmons is only possible by particle impact [55, p. 15-17].

In the study presented in Chap. 7 , the propagation of surface plasmon polaritons is investigated. Hence in the following, the dispersion and excitation of surface plasmon polaritons is discussed.

\subsection{Propagating surface plasmon polaritons}

Surface plasmon polaritons are waves which occur at metal-insulator interfaces and consist of an electron density wave below the metal surface and an electric field oscillation above the metal surface as depicted in Fig. 4.1. From the continuity conditions of electromagnetic fields at metal-insulator boundaries (cf. Eqn. 2.1 and 2.2), the dispersion 


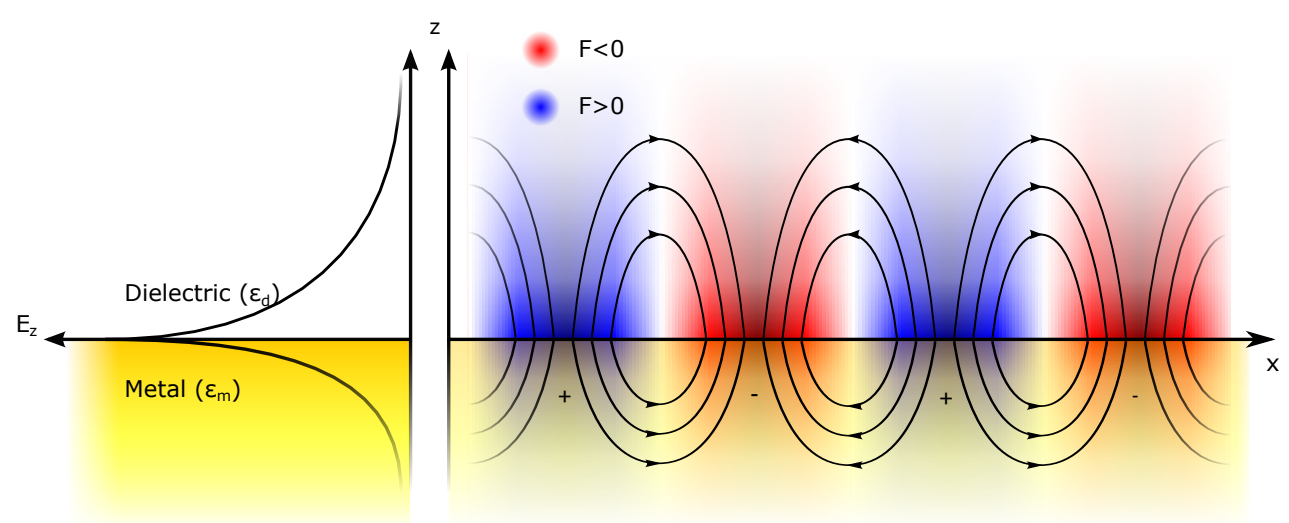

Figure 4.1: Sketch of the electric field decay (left) of a surface plasmon with increasing distance from the metal-dielectric interface. The distribution of the electric field in direction of the interface is illustrated on the right side.

relation of surface plasmons can be deduced [55, p. 26]

$$
\beta=k_{0} \sqrt{\frac{\varepsilon_{m} \varepsilon_{d}}{\varepsilon_{m}+\varepsilon_{d}}}=k_{0} n_{p}
$$

The propagation constant $\beta=k_{x}$ is the plasmon wave vector in propagation direction, $n_{p}$ is the plasmon refractive index, and $\varepsilon_{m}$ and $\varepsilon_{d}$ are the frequency-dependent dielectric permittivities for the metal and the insulator half-spaces (cf. Fig. 4.1). Surface plasmons exist only in transverse magnetic form (TM polarization), i.e., the direction of the magnetic field is perpendicular to the propagation direction of the plasmon.

The dispersion relation (Eqn. 4.4) for a metal-air interface, depicted in Fig. 4.2, shows two branches of possible excitation frequencies with a gap inbetween, where $\beta$ becomes purely imaginary. The high frequency branch represents radiative modes for $\omega>\omega_{p}$, where the metal becomes transparent. For small wave vectors, the dispersion relation is close to the light line and the electric field is extended over multiples of the wavelength into the air. In this regime, the SPPs are named Sommerfeld-Zenneck waves. In the limit of the wave vector going to infinity, the group velocity approaches zero and the surface plasmon frequency $\omega_{s p p}$ is reached asymptotically [55, p. 28]:

$$
\omega_{s p p}=\frac{\omega_{p}}{\sqrt{1+\varepsilon_{d}}} .
$$

With increasing distance from the metal-insulator interface, the electric field of the SPPs decreases exponentially.

In contrast to propagating surface plasmon polaritons, localized plasmons are spatially confined excitations at nanostructures smaller or comparable to the wavelength of the optical driving field. Due to the structure's small radius of curvature, they can be excited directly by the incident light. Localized plasmon polaritons can contribute to the field enhancement of nanostructures, e.g., at apex of NIR-excited gold nanotips.

The dispersion relation depicted in Fig. 4.2 shows that light does not couple directly to 


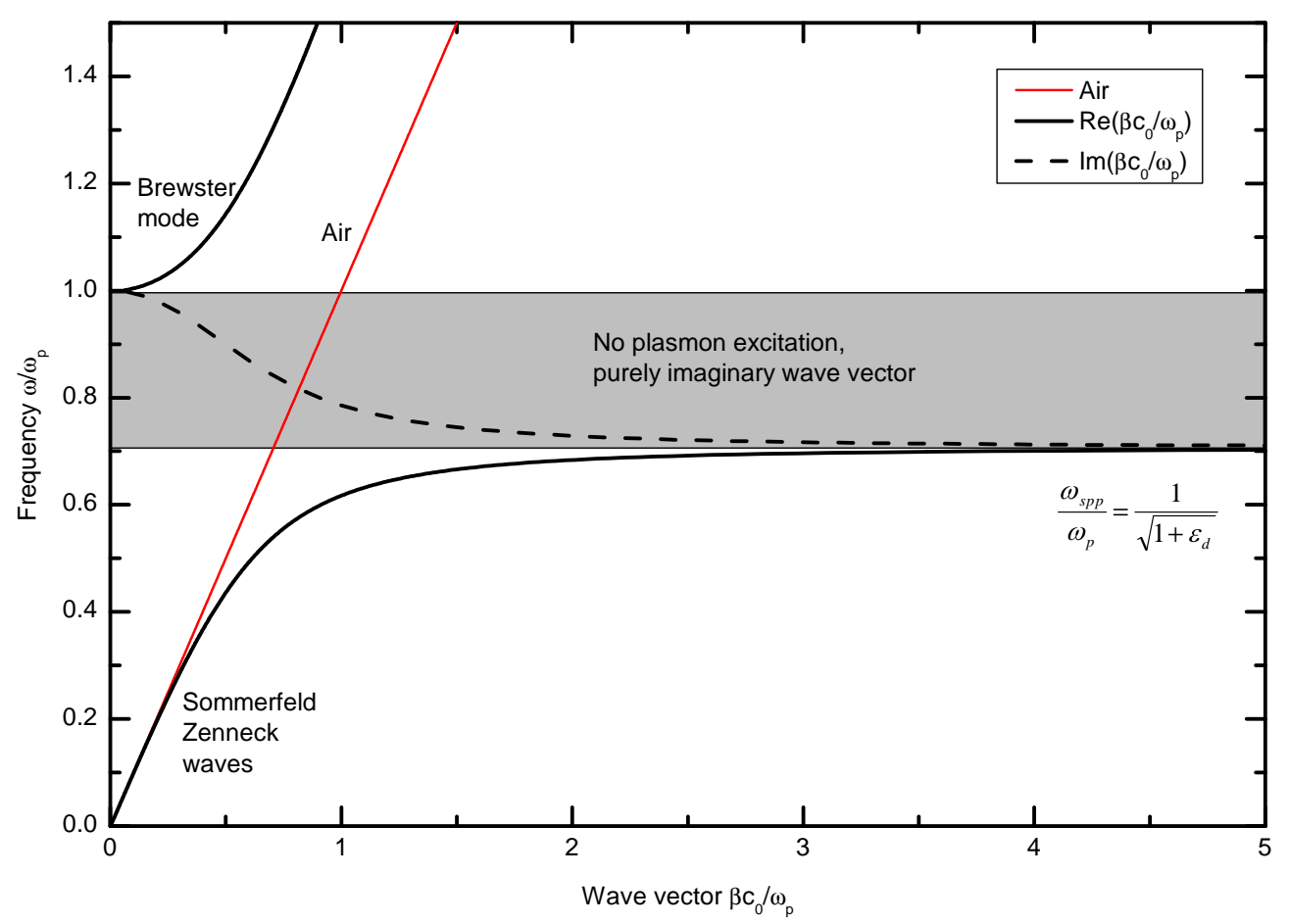

Figure 4.2: Plasmon dispersion relation without damping for a metal-air interface according to the Drude model.

propagating plasmon polaritons. For their excitation, the required energy-momentum conservation can be reached by a modification of the incident light or the surface structure as described in the following.

\subsection{Plasmon excitation on planar surfaces}

In this Section, a short introduction to the most common excitation schemes for surface plasmons, namely particle impact, prism coupling and grating excitation, is given.

A possibility to overcome the challenge of matching the dispersion relation of light and plasmons to excite surface plasmons is to employ particle impact [55, 58]. Electron energy loss spectroscopy (EELS) can be used for the excitation of bulk plasmons as well as for the generation of surface plasmons, also on metal nanotapers. For example, B. Schröder et al. [59] investigated plasmon modes at gold nanotapers using EELS. Depending on the opening angle of the tip, a standing wave pattern created by superimposing the incoming plasmon with its reflection from the apex and its higher order azimuthal modes can be detected [60,61].

For the excitation of plasmons by light, the in-plane component of the incident wave vector $k_{x}$ has to match to the propagation constant $\beta$. As depicted in Fig. 4.2, the dispersion relation of plasmons ( $\omega<\omega_{p}$, bound modes) is located right from the light line, i.e., $\beta>k$. Changing the angle of incidence $\theta$ (cf. Fig. 4.3) from grazing incidence reduces the component in direction of the interface even further: $k_{x}=k \sin \theta$. However, 
(a)

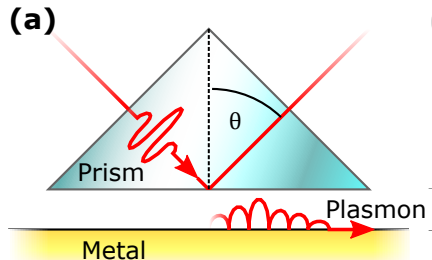

(b)

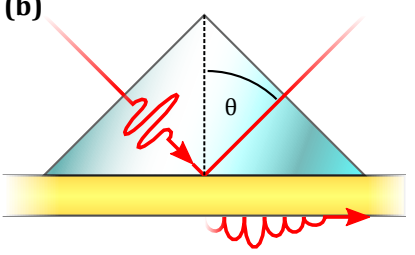

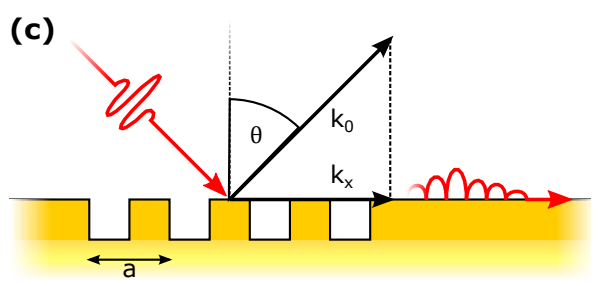

Figure 4.3: Schemes for plasmon excitation. (a), (b) Prism coupling in Otto (a) and Kretschmann (b) configuration. (c) Grating coupling.

the effective wave vector can be increased by using the evanescent wave of an additional dielectric medium.

This method, called prism coupling, requires three different materials: Two insulators of different dielectric function and a metal. Typically, one of the insulator materials is air and the plasmon is generated at the metal-air interface. The light is coupled into a prism in an angle of total internal reflection and the $\mathrm{x}$-component of the evanescent wave vector is determined by the dielectric properties of the prism: $k_{x}=k \sqrt{\varepsilon} \sin \theta$. For suitable dielectric functions $\varepsilon$, this $k_{x}$ matches to the plasmon dispersion relation, and the evanescent wave couples into the plasmon, attenuating the reflected beam. Prism coupling is typically implemented in
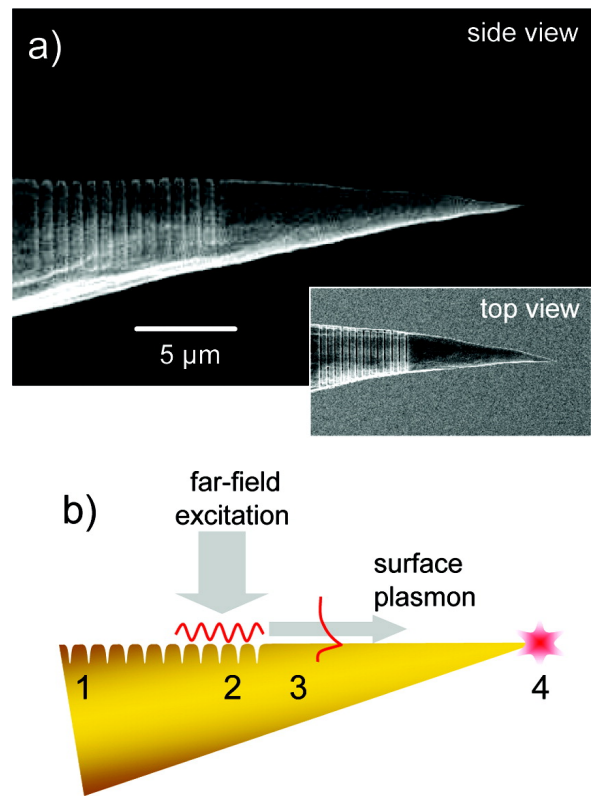

Figure 4.4: Plasmon excitation via grating coupling on a nanotip. (a) Scanning electron microscope image of a tip with a grating coupler on the shaft. (b) Excitation scheme (Reprinted with permission from [62]. Copyright (2007) American Chemical Society).
Kretschmann [63] or in Otto [64] configuration as depicted in Fig. 4.3(a),(b). In Otto configuration, the prism does not touch the metal facilitating measurements of the surface quality.

In the study presented in Chap. 7, light is coupled into an optical grating on the shaft of a gold nanotip to excite the plasmons. The in-coupling of surface plasmons at a grating was first observed by $R$. W. Wood in 1902 as an anomaly in the reflected spectrum of a diffraction grating ([65], "Wood anomalies"). On metal nanotapers, grating coupling was first implemented by $C$. Ropers et al. [62] (see Fig. 4.4). The mechanism behind grating coupling can be understood in terms of diffraction. The in-plane component of the incident wave vector has to fulfill the Laue condition for positive interference along the grating [55, p. 45]:

$$
\beta=k \sin \theta \pm m g
$$

with the diffraction order $m \in \mathbb{N}$, the grating constant $g=2 \pi / a$, and the grating period $a$. For the excitation of surface plasmon polaritons, at least a part of the polarization 

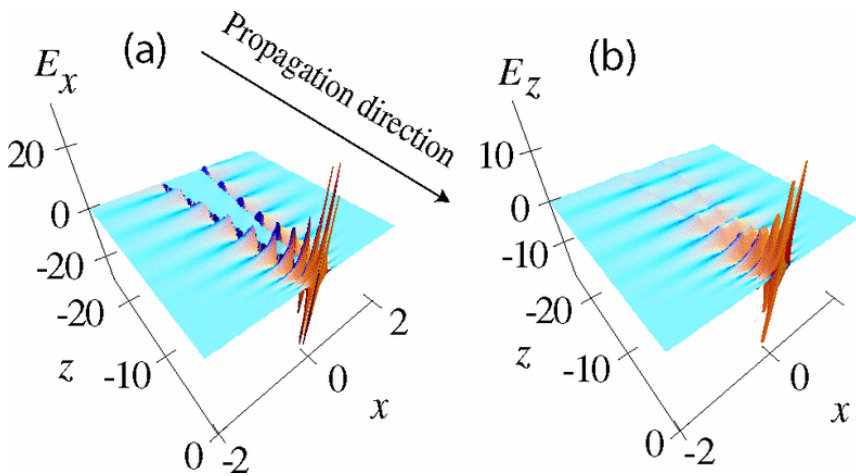

Figure 4.5: Electric field of a plasmon propagating along a nanotip shaft. (a) $x$-component of the field. (b) $z$ -

2 component of the field (Reprinted Figure with permission from [69] Copyright (2004) by the American Physical Society).

has to point in perpendicular direction to the grating.

Using a grating directly milled into the tip shaft for plasmon excitation is an efficient and robust method that is applicable in many different experimental configurations. An alternative approach is, e.g., the in-coupling of plasmons at nanodot patterns on a flat substrate, and the plasmon propagates to a tapered nanostructure attached so the substrate [66].

The history and the physics of grating coupling is described in detail, e.g., in the $\mathrm{PhD}$ thesis of A. P. Hibbins [67] and of A. N. Sprafke [68].

\subsection{Adiabatic nanofocusing}

Plasmon modes propagating along the shaft of a nanotaper towards the apex are confined to the decreasing radius of the nanometric cone, facilitating foci well below the diffraction limit. This process is called adiabatic nanofocusing [69]. The electric field of such a nanofocused plasmon mode is depicted in Fig. 4.5. At the apex of the tip, the propagating plasmon mode is scattered into the far field, and for high plasmon energies, electrons can be emitted. Such a nanometric light or electron source is highly advantageous for background-free optical near-field scanning and electron probe techniques.

For small taper radii $r$ below the vacuum wavelength $\left(k_{0} r \ll 1, k_{0}\right.$ vacuum wavenumber), the plasmon refractive index depends on the taper radius. Applying the eikonal approximation, i.e., the assumption that the refractive index does not change significantly over distances in the range of the wavelength, and the boundary conditions at metal-insulator interfaces, the refractive index $n_{p}$ for the plasmon as a function of the taper radius $r$ is given by [69]:

$$
n_{p}(r) \approx \frac{1}{k_{0} r} \sqrt{-\frac{2 \varepsilon_{d}}{\varepsilon_{m}}}\left[\ln \sqrt{-\frac{4 \varepsilon_{m}}{\varepsilon_{d}}}-\gamma_{E}\right]^{-1} .
$$

In this equation, $\gamma_{E}=0.57721$ is the Euler-Mascheroni constant and $k_{0}$ is the vacuum wavenumber. The refractive index increases with decreasing cone radius, which corresponds to a deceleration of the propagating plasmon. At the apex, the adiabatic approximation of a slowly varying taper radius is not valid anymore. Here, the plasmon 
mode scatters into the far-field and energy is transferred from the plasmon mode to the metal, leading to a heating and an extension of the nanotip.

Theoretical studies about adiabatic nanofocusing for different focusing conditions can be found, e.g., in the Refs. [69-71]. 



\section{Chapter 5}

\section{Experimental methods}

This Chapter details the experimental setup of THz near-field streaking at metal nanotips that was used to obtain the results presented in the following Chaps. 6 and 7 . First, an overview of the complete beam path of the setup is given with a special attention to the core of the experiment, i.e., the two-color photoemission at the apex of the tip as introduced in Sec. 3.2 and Ref. [15]. Subsequently, four topics that are essential for the successful implementation of the experiment are discussed:

- The generation of the $\mathrm{THz}$ pulses in a light-induced air plasma using the AC bias method,

- the capture of the electron energy spectra by a time-of-flight spectrometer,

- the calibration procedure to compute the streaking spectrograms from the raw data,

- and the measurement of the $\mathrm{THz}$ transient by electro-optic sampling.

\subsection{Experimental setup}

In Figure 5.1, the beam path of the streaking experiment is schematically depicted. The setup is operated by a titanium-sapphire amplifier system from Coherent, based on a "Legend Elite" amplifier, a "Vitesse" oscillator and an "Evolution" pump laser. The output power of the amplifier system amounts to $4 \mathrm{~W}$ at $1 \mathrm{kHz}$ repetition rate, $800 \mathrm{~nm}$ wavelength, and 50 fs pulse duration. To implement the streaking experiment, only a fraction of the output power of $1.7 \mathrm{~W}$ is directed into the beam path in Fig. 5.1.

The actual streaking experiment is conducted in a vacuum chamber, where the $\mathrm{THz}$ transients and NIR pulses are focused collinearly onto the apex of an electrochemically etched gold nanotip (for the manufacturing process see, e.g., Ref. [72]). An off-axisparabolic mirror with a focal length of $25.4 \mathrm{~mm}$ directs the $\mathrm{THz}$ beam to the apex. 


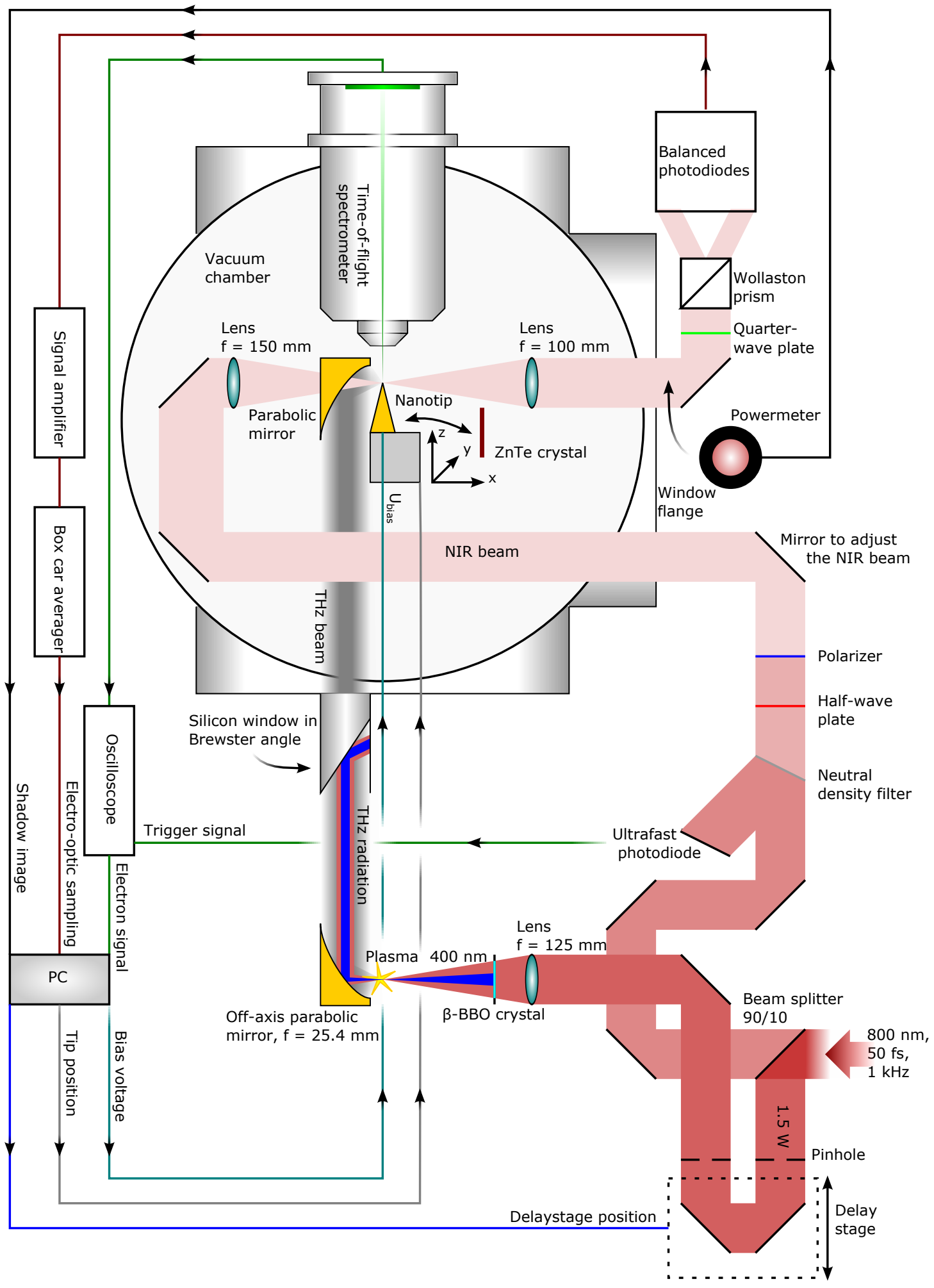

Figure 5.1: The beam path used for $\mathrm{THz}$ streaking on metal nanotips including the measurement control and the data acquisition scheme. 
The NIR beam is guided by a lens (150 mm focal length) through a small hole in the parabolic mirror.

For an accurate positioning, the nanotip is fixed on a holder that is attached to a $3 \mathrm{D}$ translation stage (a combination of three compact micro translation stages "M111" from $P I$, travel range $15 \mathrm{~mm}$ ). The tip holder is designed for four nanowires that can be shifted in the combined focus of the THz and the NIR beams. To measure the incident $\mathrm{THz}$ transient that generates the near-field at the tip apex, electro-optic sampling measurements are conducted using a ZnTe crystal attached to the tip holder and moved into the focus.

The kinetic energy of the photoelectrons is detected by a time-of-flight spectrometer (TOF) "ETF 11" from STEFAN KAESDORF. The nanotip apex is positioned in a distance of $3 \mathrm{~mm}$ from the entrance of the TOF, which is the optimal working distance of the device. By tilting the mirror in front of the window flange (cf. Fig. 5.1), the $800 \mathrm{~nm}$ beam is aligned to the tip apex using a shadow image of the tip and a 2D map of the NIR-induced photocurrent. The shadow image is recorded by measuring the average NIR beam power behind the tip as a function of the stage position. The resulting shadow image shows the tip shape convoluted with the form of the NIR focus. The intensity of the NIR photoemission pulse ( $1 \mathrm{kHz}$ repetition rate, $50 \mathrm{fs}, 800 \mathrm{~nm})$ is adjusted by a set of neutral density (ND) filters and is fine-tuned by the combination of a half-wave plate and a polarizer. To avoid intensity-induced damages of the tip and the ZnTe crystal, the experiments are performed below $100 \mu \mathrm{W}$ average NIR input power. The back-reflection of the ND filter is detected by an ultrafast photodiode and used as a trigger signal for the data capturing.

The delay of the two pulses is controlled by a translation stage (Newport " $M$-VP-25XL", $25 \mathrm{~mm}$ travel range, minimum step size $10 \mathrm{~nm}$ ) that is included in the beam path used for the generation of the $\mathrm{THz}$ pulses. The voltage signal from the TOF is recorded by an oscilloscope that is operated via LabView.

\subsection{Generation of the THz transients using the AC bias method}

The THz transients are generated using the so-called AC bias method (Review, e.g., in Ref. [73]). This method was introduced by T. Cook et al. in 2000 [74] and is based on the electron motion in a two-color plasma. Figure 5.2 depicts a scheme for the generation of $\mathrm{THz}$ pulses by the AC bias method: Intense NIR pulses are focused by a lens through a $\beta$ barium borate (BBO) crystal (orientation 29.2 , optimized for phase-matching) for the generation of the second harmonic. In the combined focus of the fundamental and the second harmonic, a light-induced air-plasma is ignited. The electrons in the plasma are accelerated in a two-color field and radiate in the $\mathrm{THz}$ range. This process can be described by four-wave mixing, and the spectrum of the $\mathrm{THz}$ transient results from the spectral distribution of the fundamental and its second harmonic. The intensity and 


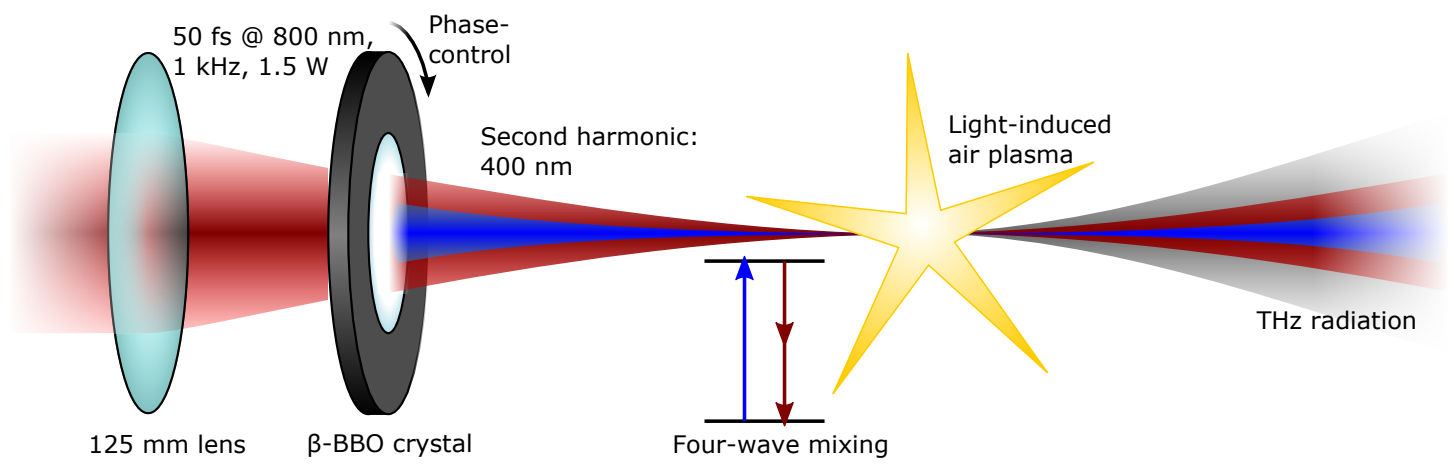

Figure 5.2: Setup for the generation of $\mathrm{THz}$ radiation using the $\mathrm{AC}$ bias method. The specific values for the incident NIR beam and the focusing lens apply to the setup implemented in our beam path.

the carrier-envelope phase (CEP) of the $\mathrm{THz}$ pulses depend on the rotation angle of the BBO crystal and the distance of the crystal to the focus. The minimum distance between the crystal and the focus is determined by the damage threshold of $\beta$ barium borate.

The AC bias method is especially suitable for streaking experiments, since it allows for a tuning of the carrier-envelope phase by the angle of the BBO crystal. Switching the polarity of the $\mathrm{THz}$ transient can be employed for a reconstruction of the full near-field waveform as described in Sec. 3.2.1. The collinearity of the THz beam with the $800 \mathrm{~nm}$ fundamental facilitates the adjustment of the $\mathrm{THz}$ beam.

The $\mathrm{THz}$ beam enters the vacuum chamber through a silicon window in the Brewster angle, which enables the complete transmission of the horizontally polarized component of the $\mathrm{THz}$ beam. At the silicon window, the NIR beam and its second harmonic are reflected and blocked.

\subsection{Time-of-flight spectrometer}

In a time-of-flight (TOF) spectrometer, the kinetic energy of the electrons is deduced from their propagation time $t$ to the detector within the TOF. If the electrons are not accelerated in the drift path, their energy is given by the equation for uniform motion:

$$
E=\frac{m_{e} s_{p r o p}^{2}}{2 t^{2}}
$$

Here, $E$ is the electron energy and $s_{\text {prop }}$ the propagation distance. The energy resolution of the electron spectra depends nonlinearly on the time resolution of the signal processing system and is higher for low energies. The time-resolution of our system, the "ETF11" electron spectrometer from STEFAN KAESDORF (cf. Fig. 5.3) in combination with a $1 \mathrm{GHz}$ oscilloscope ("Agilent infinium DSO9104H"), is measured to be around $3.2 \mathrm{~ns}$. Hence, the energy resolution is limited to about $1 \mathrm{eV}$ at an electron energy of 10-15 eV. 


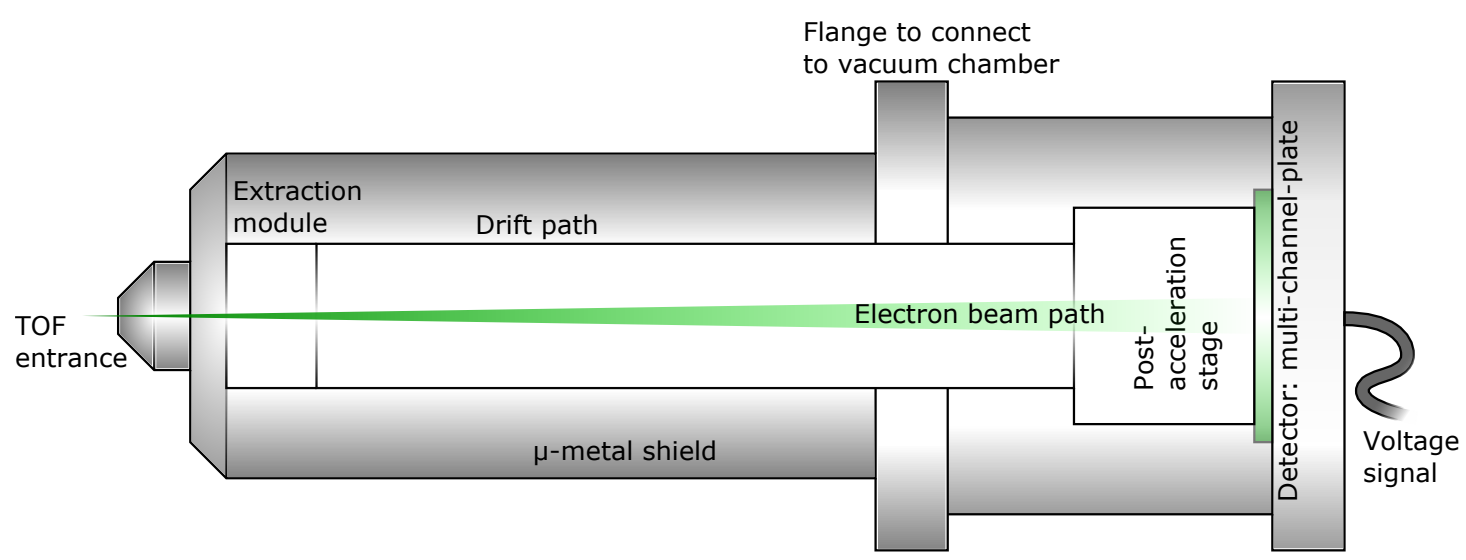

Figure 5.3: Sketch of the STEFAN KAESDORF time-of-flight spectrometer "ETF 11" for electron spectroscopy.

For energies below $10 \mathrm{eV}$, the detection efficiency strongly decreases, and the spectral resolution is limited by artefacts.

A lens voltage up to $1 \mathrm{kV}$ directs the electrons into the spectrometer. The spectrometer has a drift path with a length of $294 \mathrm{~mm}^{1}$, where a voltage in the range of $\pm 180 \mathrm{~V}$ can be applied. This drift voltage is employed to increase the energy resolution by manipulating the energy of the electrons before they are entering the drift path. At the end of the drift path, a multi-channel plate (MCP) detects the electrons and transmits a short voltage peak to the oscilloscope.

The detection efficiency of the TOF depends on the kinetic energy of the electrons and is adjusted by the lens voltage. As a rule of thumb, the detection efficiency is optimal for a lens voltage which is four to five times higher than the electron energy in $\mathrm{eV}$. At this voltage, the electron beam covers the entire detection area and the response function is relatively energy-independent. If the electron energy is too low in comparison to the lens voltage, the electron beam is overly focused and the detection efficiency of the MCP is limited by its recovery time. If the electron energy is too high compared to the lens voltage, not all electrons reach the detector due to the divergence of the electron beam. The voltage signal from the MCP is transferred to the oscilloscope, which is triggered by the laser excitation pulse taken from a back-reflection of the neutral density filter. This trigger signal appears in a constant time difference with respect to the emission time and serves as a reference for the data evaluation. The oscilloscope summarizes the voltage traces to an arrival time histogram, counting the voltage peaks as a function of the detection time. The histograms are read out by a LabView routine and saved by a LabView program.

To calibrate the raw data, a series of histograms is recorded for increasing bias voltage without $\mathrm{THz}$ acceleration.

\footnotetext{
${ }^{1}$ The total propagation distance amounts to $404.5 \mathrm{~mm}$, including the working distance and the post acceleration stage.
} 

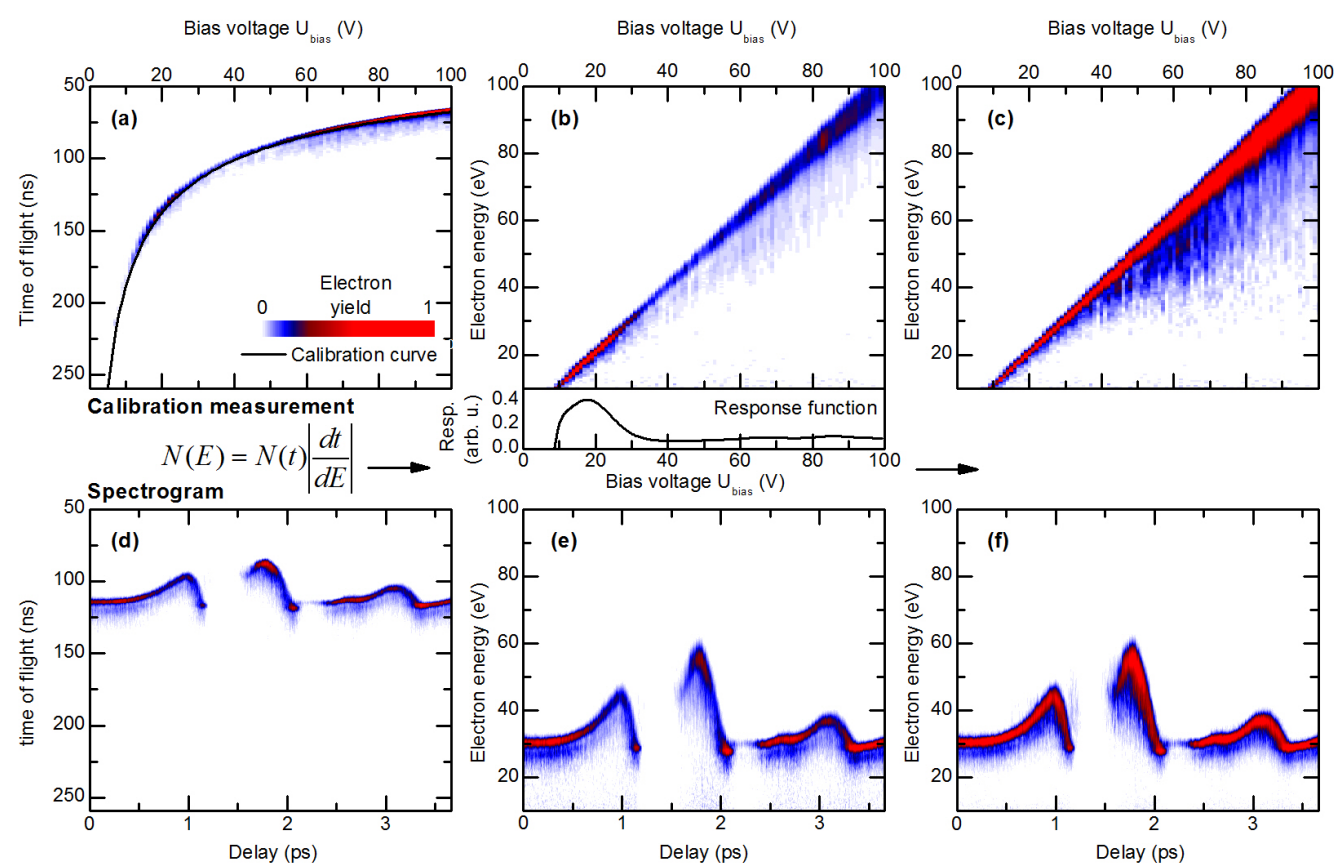

Figure 5.4: Calibration of the electron energy spectra (lens voltage $600 \mathrm{~V}$ ). (a) Raw data of the calibration measurement and a calibration curve provided by STEFAN KAESDORF. (b) Calibration of the measurement in (a) using the calibration curve. The electron yield is preserved by employing $N(E)=|d t / d E| N(t)$. The plot below (b) depicts the response function, i.e., the maximum amplitude of the electron yield in (b). (c) The calibrated measurement from (b) divided by the response function. In (d)-(f), the calibration procedure from $(\mathrm{a})-(\mathrm{c})$ is applied to an exemplary measurement $\left(U_{\text {bias }}=-30 \mathrm{~V}\right)$, which is part of the series discussed in Chap. 6.

\subsection{Data processing}

The LabView program employed for the measurements controls the initial and the final position of the delay stage, the delay step size, the acquisition time, the bias voltage and the number of measurement repetitions. Both an increase of the recording time and of the repetition loops improve the data quality but only repeated measurements of the same delay range help to distinguish real physical effects in the streaking spectrograms from effects arising from a degradation of the tip.

The final spectrograms are computed in three steps. First, an averaged spectrogram is computed from the repeated measurements. In a second step, the calibration measurement is evaluated to extract the response function and compared with the calibration curve provided by STEFAN KAESDORF to determine the emission time of the electrons. These calibration curves are required to consider small deviations from the relation given in Eqn. 5.1 due to the lens voltage. In the third step, the measured spectrograms are calibrated using the emission time and the response function obtained from the previous steps. The calibration procedure is depicted Fig. 5.4 for a calibration 
measurement (cf. Fig. 5.4 (a)-(c)) and an exemplary spectrogram (cf. Fig. 5.4 (d)-(f)). The calibration data from STEFAN KAESDORF contain the kinetic energy as a function of time. From the maxima of the calibration measurement, an experimental calibration curve is extracted, which has to be temporally shifted to match to the theoretical values (cf. Fig. 5.4(a)), i.e., the emission time of the electrons is determined. To preserve the electron yield, the energy derivative of the propagation time has to be considered for the computation of the electron energy spectra $N(E)$ from the arrival time histograms $N(t)$ (applied in Fig. 5.4 (b) and (e))

$$
N(E)=N(t)\left|\frac{d t}{d E}\right|=N(t) \sqrt{\frac{m_{e}}{8}} s E^{-3 / 2} .
$$

If a drift voltage is applied to measure a spectrogram, a calibration measurement with the same drift voltage is required, and the measured calibration curve has to be shifted in energy to match to the theoretical calibration data.

The variations of the detection efficiency due to the lens voltage are considered by a response function (Fig. 5.4(b), below), which is extracted from the maximum electron yield of the measured calibration curve. The measured electron yield is normalized to the response function to gain a more realistic estimate of the electron yield (see. Fig. 5.4 (c) and (f)).

The assumption behind this procedure is that the Schottky effect is negligible and the photocurrent stays constant during the increase of the negative bias voltage. In the simulation of the spectrograms, the Schottky effect due to the static bias is relatively small, justifying our assumption.

\subsection{Electro-optic sampling}

The THz transients are measured by electro-optic sampling (EOS). This technique is based on the linear electro-optic effect and allows for the phase-resolved detection of the $\mathrm{THz}$ pulses, including a quantitative measurement of the field strength.

A setup for electro-optic sampling measurements is depicted schematically in Fig. 5.5. The horizontally polarized $\mathrm{THz}$ beam is focused together with the NIR beam in $45^{\circ}$ polarization in a (110) ZnTe crystal (thickness $l=500 \mu \mathrm{m}$ ). The refractive index of the electro-optic crystal depends linearly on the electric field (here: THz field) applied to the crystal (linear electro-optic effect). Thus, the horizontally polarized component of the NIR beam experiences a different refractive index than the vertically polarized component. After passing through the ZnTe crystal, the NIR beam is elliptically polarized with one half-axis pointing in $45^{\circ}$ direction measured from the horizontal plane (see Fig. 5.5, step 2). A quarter-wave plate in $45^{\circ}$ angle changes the polarization angle according to the ellipticity of the incident light. Behind the quarter-wave plate, the NIR beam is polarized linearly, again, but the polarization direction is altered (Fig. 5.5, step 3). The 


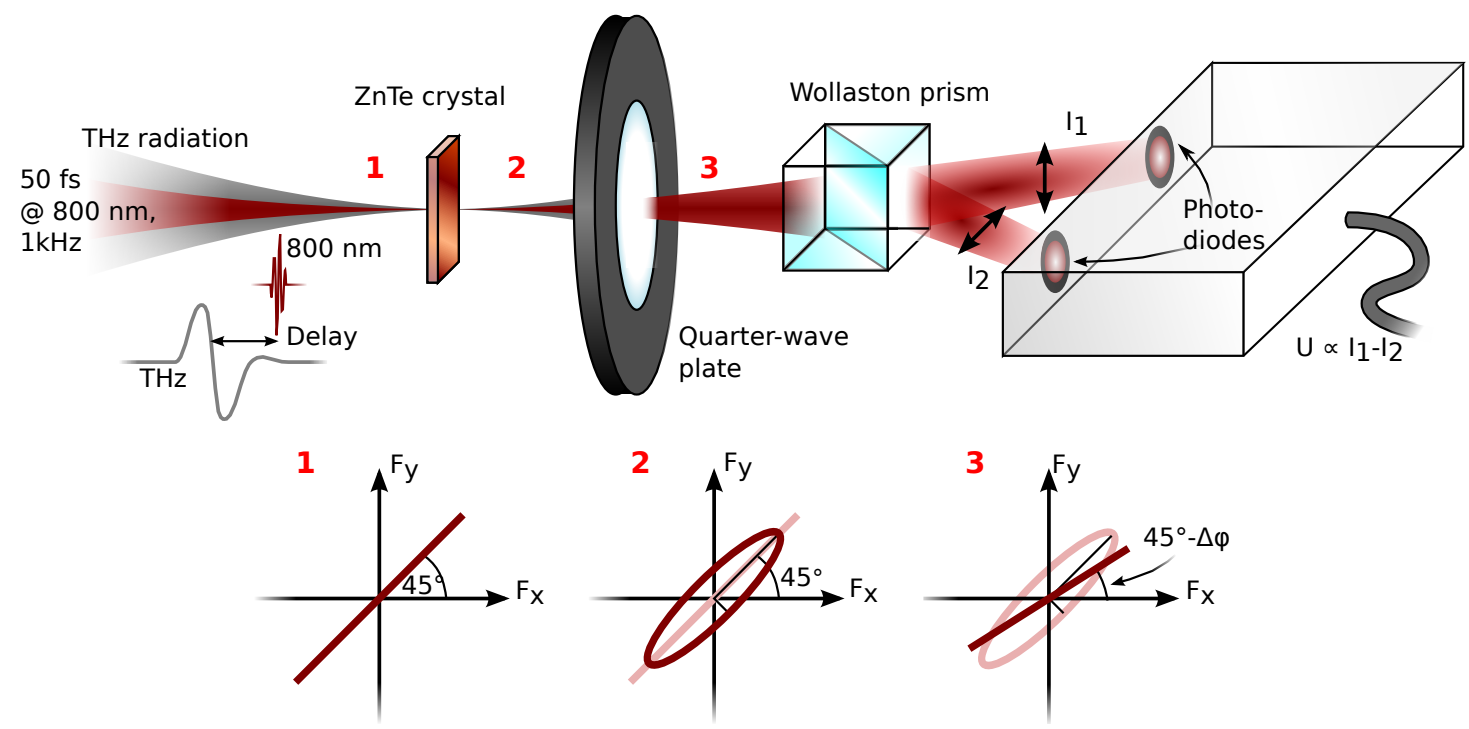

Figure 5.5: Setup for the measurement of the THz transients by electro-optic sampling. The field-induced rotation of the polarization direction is depicted in the steps 1-3.

THz-induced rotation of the polarization direction is measured as the difference voltage signal $U$ of two balanced photodiodes, and the $\mathrm{THz}$ field strength is computed as:

$$
F=\frac{\lambda}{\pi \ln _{g}^{3} r_{41}} \arcsin \left(\frac{U}{k_{c a l}}\right) .
$$

In this equation, $k_{c a l}$ is a calibration constant, $n_{g}$ is the group refractive index of $\mathrm{ZnTe}$ at $800 \mathrm{~nm}$ and $r_{41} \approx 4 \mathrm{pm} / \mathrm{V}$ is the linear electro-optic coefficient in the THz range [75]. For small $U / k_{\text {cal }}$, the voltage signal is proportional to the field strength. The measurements are calibrated by a rotation of the quarter-wave plate as depicted in Fig. 5.6(a). The maximum amplitude $a_{\max }$ of the sinusoidal calibration curve is related to the calibration parameter by $a_{\max }=k_{\text {cal }} / 2$.

For the data evaluation, we employ a frequency-dependent response function (Fig. 5.6(b), lower plot) that is taking into account

- the absorption in the crystal,

- the effect of the NIR pulse duration limiting the detection of higher frequency components,

- and the reflection of the $\mathrm{THz}$ beam at the entrance facet of the crystal due to the discontinuity of the refractive index.

The frequency-dependent refraction characteristics are taken from Ref. [76].

ZnTe exhibits a strong electro-optic response, which limits the maximum detectable field strength to $\approx 85 \mathrm{kV} / \mathrm{cm}$ (with frequency-dependent response function $\approx 200 \mathrm{kV} / \mathrm{cm}$ ) for a $500 \mu \mathrm{m}$ thick crystal plate. This restriction is given by the maximum of the sine function in Eqn. 5.3. At a frequency of $5.3 \mathrm{THz}$ [75], ZnTe exhibits a phonon resonance, which limits the detection range to frequencies below $3 \mathrm{THz}$. An alternative 

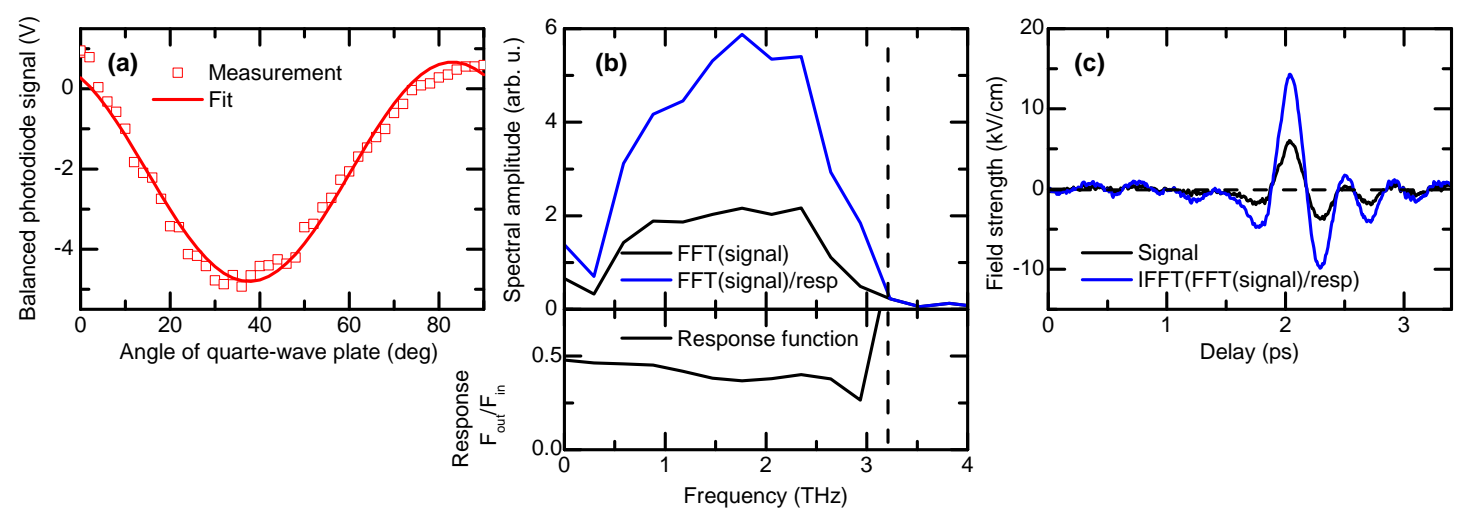

Figure 5.6: Calibration of the electro-optic sampling measurements. (a) Signal of the balanced photodiodes as a function of the quarter-wave plate angle. This measurement allows to determine the calibration constant $k_{c a l}$. (b) Fourier transform of the measurement before and after the frequency-dependent evaluation using the response function depicted below. (c) Calibrated measurement with and without taking the response function into account.

material could be, e.g., GaP which is characterized by a lower electro-optic coefficient $\left(r_{41} \approx 1 \mathrm{pm} / \mathrm{V}\right)$ and a phonon resonance at $11 \mathrm{THz}$. An extensive review of electro-optic sampling in ZnTe and GaP is given by S. Casalbuoni et al. in Ref. [75]. 



\title{
Chapter 6
}

\section{Phase space manipulation of free-electron pulses from metal nanotips using combined terahertz near fields and external biasing}

\author{
L. Wimmer, O. Karnbach, G. Herink, and C. Ropers \\ Physical Review B 95 165416, April 2017 \\ DOI: 10.1103/PhysRevB.95.165416 \\ (C)2017 American Physical Society)
}

We present a comprehensive experimental and numerical study of photoelectron streaking at metallic nanotips using single-cycle terahertz $(\mathrm{THz})$ transients and a static bias voltage as an external control parameter. Analyzing bias voltage dependent streaking spectrograms, we explore the THz-induced reshaping of photoelectron energy spectra, governed by the superimposed static field. Numerical simulations are employed to determine the local field strengths and spatial decay lengths of the field contributions, demonstrating electron trajectory control and the manipulation of the phase space distributions in confined fields with both dynamic and static components.

\subsection{Introduction}

Progress in time-resolved experimental techniques is driving an increased understanding of the ultrafast electronic, structural and spin response of bulk materials, surfaces, and nanostructures. Ultrafast electron diffraction and microscopy [77-84] provide unique 
insights into the dynamics of nanoscale processes, with broad applications ranging from the study of structural phase transitions $[78,85]$ to free-electron quantum optics [86, 87]. The capabilities of these techniques strongly depend on the quality of the electron source, including its spatial beam properties and pulse duration. While the transverse beam coherence is greatly enhanced by the use of nanoscale electron sources or tailored photoemission processes [14, 80, 88-99], ultrashort pulse durations are achieved either by minimizing propagation distances from the source to the sample $[78,80]$, or by active means using time-dependent electric fields [13, 14, 100-103]. Electron pulse compression in microwave cavities is an established technique to produce ultrashort pulses of high bunch charge [100, 101].

At optical frequencies, inelastic near-field interactions with free electron beams [90, 104, $105]$ were recently applied in a quantum-coherent manner [86, 87], creating the possibility of forming attosecond electron pulse trains $[86,106]$. Between the optical and microwave domains, intense phase-stable mid-infrared and Terahertz waveforms currently promote access to nonlinear and strong-field phenomena [107, 108], including Terahertz high-harmonic generation in semiconductors [109] and ultrafast scanning tunneling microscopy $[2,110,111]$. Moreover, mid-IR photoemission studies at metal nanotips [16, 94, 95] demonstrate the prospects for the manipulation of free-electron beams, yielding field-driven acceleration with unique sub-cycle dynamics arising from the nanoscale field confinement. In strong-field photoemission from nanostructures [16, 45, 91, 112-117], the emission and acceleration processes are driven by the same field and are thus closely coupled. Inducing both processes by independent fields, streaking spectroscopy allows for a characterization of electromagnetic fields by their impact on photoelectron spectra $[5,6]$. Originally developed in attosecond science and applied to gas phase targets and planar surfaces, the concept was recently transferred to optical nanostructures $[15,44$, 52], demonstrating unique features offered by streaking in spatially-confined fields. In particular, THz near-field control and sub-cycle streaking of photoelectron emission from metal nanotips were realized [15], followed by demonstrations of THz-induced tunneling emission [17, 118, 119], electron acceleration in waveguides [120-123], and electron pulse compression and deflection in resonant structures [14, 42, 124-126]. In the subcycle regime of electron acceleration, the energy gain in near-field streaking sensitively depends on the time spent by the electrons within the confined field [15, 16, 40, 42, 44, 127]. This suggests that an additional static field driving the electrons out of the localized streaking region may afford additional control over the electron dynamics.

Here, we experimentally demonstrate that an external bias voltage applied to a nanoscale photoemitter offers a powerful means of manipulating the electron dynamics in $\mathrm{THz}$ near-fields. The basic experimental concept is illustrated in Fig. 6.1. We conduct THz streaking spectroscopy of femtosecond photoelectron pulses emitted from the apex of a gold nanotip in the presence of a variable bias field, as depicted in Fig. 6.1(a). The effect of the static bias on the electron dynamics is illustrated in Figs. 6.1(b)-6.1(e). Generally, the negative bias field draws the electrons towards the detector [cf. Fig. 6.1(b)]. For 
(a)

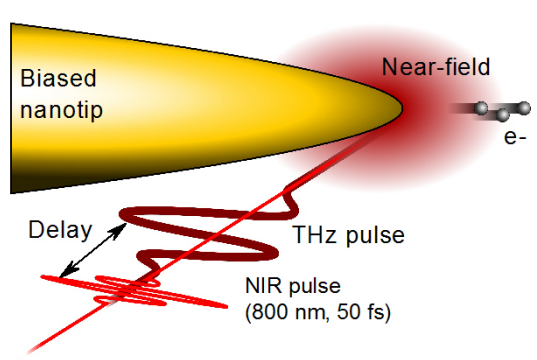

(b) No bias

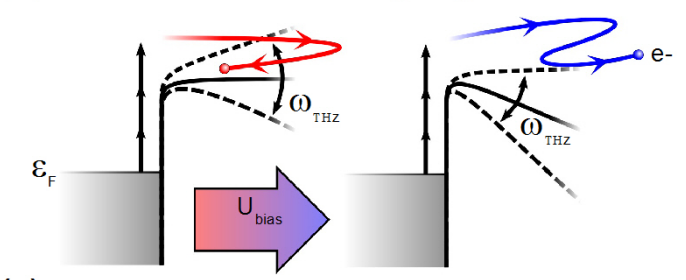

(c)

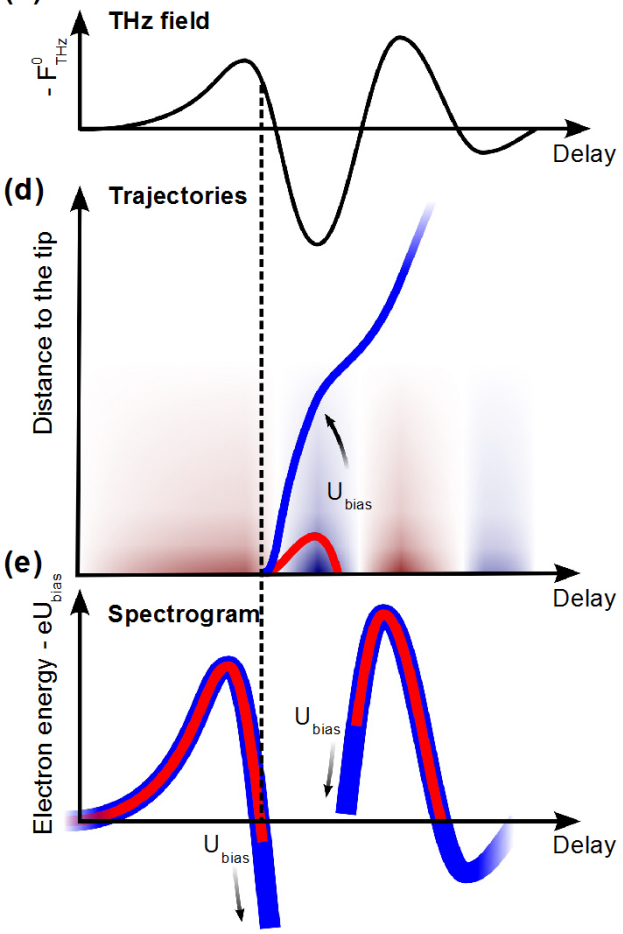

Figure 6.1: Basic principle of $\mathrm{THz}$ streaking at biased nanotips. (a) Experimental streaking scheme. (b) Emission and propagation of photoelectrons in the absence of a bias (left, red trajectory) and with a high negative static bias (right, blue trajectory). The static field bends the potential, allowing for the electrons to leave the near-field. (c) $\mathrm{THz}$ surface electric field. (d) Electron trajectories without external bias (red trajectory) and with additional acceleration by a static bias field (blue trajectory). The background color indicates the $\mathrm{THz}$ electric field. (e) The sketched red and blue streaking spectrograms illustrate the impact of an increasing bias voltage.

certain emission phases within the THz transient [cf. Fig. 6.1(c)], this additional acceleration strongly influences the electron trajectories and the photocurrent. For example, in emission phases of otherwise suppressed photocurrent, the negative bias field allows the photoelectrons to leave the THz near-field [see Fig. 6.1(d)]. The bias-induced changes in the streaking spectrograms are illustrated in Fig. 6.2, showing drastic modifications of the time-dependent photoelectron spectra. Numerical simulations corroborating the experimental data allow for a quantitative characterization of the local field parameters of the $\mathrm{THz}$ and static fields. The analysis of ensembles of electron trajectories reveals that the phase space density distribution of the electron pulses is strongly affected by the combined action of the $\mathrm{THz}$ near-field and the bias.

Our work demonstrates the virtue of spatially confined electric fields in the $\mathrm{THz}$ range 

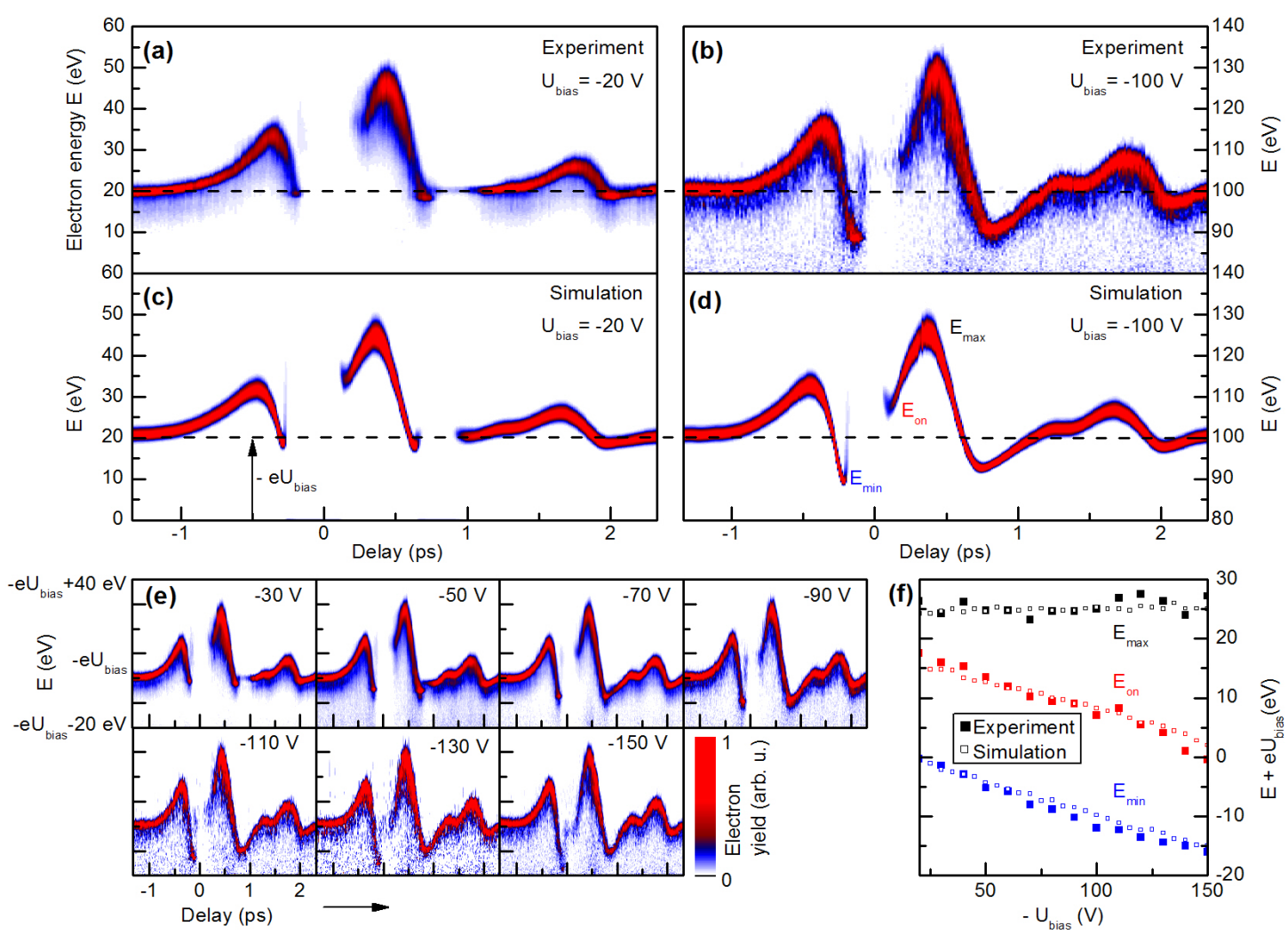

Figure 6.2: $\mathrm{THz}$ streaking spectrograms at biased metal nanotips. (a), (b) Experimental streaking spectrograms (bias voltage: -20 (a) and $-100 \mathrm{~V}$ (b)). (c) and (d): Corresponding simulations of the streaking spectrograms. (e): Extended series of streaking spectrograms recorded at bias voltages ranging from $-30 \mathrm{~V}$ to $-150 \mathrm{~V}$. (f) Extracted bias dependence of the minimum and maximum energy gain in the THz-field, and of the energy gain at the onset of the photocurrent (see labels in (d)).

for the active control of ultrashort electron pulses with additional leverage obtained by a superimposed static field.

\subsection{Experimental procedure and results}

In the experiments, 50-femtosecond near-infrared (NIR) pulses and single-cycle $\mathrm{THz}$ transients are jointly focused onto a gold nanotip [Fig. 6.1(a)]. The NIR-pulse generates photoelectrons at the tip apex by multiphoton photoemission, which are subsequently accelerated in the THz-induced near-field. A negative bias voltage $U_{\text {bias }}$ applied to the tip additionally accelerates the electrons [cf. Fig. 6.1(b)]. Kinetic energy distributions of photoelectrons are recorded with a time-of-flight spectrometer as a function of relative time-delay between the NIR and the THz pulses, resulting in streaking spectrograms as displayed, e.g., in Figs. 6.2(a), 6.2(b), and 6.2(e).

The streaking spectrograms demonstrate a strong influence of the $\mathrm{THz}$ electric field on the photoelectron emission and propagation. Before we discuss the influence of the external bias, let us first reconsider the main features of such spectrograms [see, e.g., 
Fig. 6.2(a)], some of which were covered in detail in our previous work [15-17]. The $\mathrm{THz}$ field affects both the photoemission current and the photoelectron kinetic energy. Generally, the tip exhibits a rectifying behavior, in which significant photocurrent is only observed for $\mathrm{THz}$ phases with positive force on the electrons, i.e., negative electric field, and the photocurrent is suppressed for an electric force directing the electrons to the tip [see, e.g., the current-free interval around zero delay in Fig. 6.2(a)]. Upon variation of the delay, the kinetic energy spectra trace out the overall electron acceleration induced by the locally enhanced $\mathrm{THz}$ field and the static bias. Due to the high localization and field enhancement, which is characteristic for optically induced near-fields at metal nanostructures, the delay-dependent kinetic energy mainly follows the $\mathrm{THz}$ field in the moment of emission [15].

The basic principles of field-driven electron acceleration in near-fields are characterized by a spatial adiabaticity parameter $[16] \delta=l_{f} / l_{q}$, which relates the spatial near-field decay length $l_{f}$ with the electron quiver amplitude $l_{q}=e F / m_{e} \omega^{2}$ in a (homogeneous) field of strength $F$ and at a frequency $\omega$. Field-driven, sub-cycle dynamics, in which photoelectrons leave the optical near-field directly after the emission process, correspond to $\delta \ll 1$, whereas electron dynamics governed by propagation effects are associated with a larger $\delta$. In the present case of studying the electron dynamics in a phase-resolved manner, we can identify the maxima of the kinetic energy trace with field-driven electron dynamics. While the streaking waveform is generally characterized by quasi-instantaneous electron acceleration [15], in emission phases near zero-crossings of the THz field, propagation effects are expected to have a measurable influence. Around these phases with near-zero streaking field, the electron kinetic energy sensitively depends on the propagation time within the $\mathrm{THz}$ near-field, which can be controlled by the external bias.

In order to investigate the effect of a static field on the streaking process, we systematically record a series of streaking spectrograms for bias voltages $U_{\text {bias }}$ in the range of -20 to $-150 \mathrm{~V}$, for fixed optical and $\mathrm{THz}$ excitations. The NIR beam $(40 \mu \mathrm{W}$ input power, $10 \mathrm{~mm}$ beam diameter) is focused by a $150 \mathrm{~mm}$ planoconvex lens and the $\mathrm{THz}$ beam (20 mm diameter) is focused by a parabolic mirror (focal length of $25.4 \mathrm{~mm}$ ) to about $1 \mathrm{~mm}$. All measurements presented in this work are recorded using the same nanotip with an apex radius of curvature of $20 \mathrm{~nm}$ [cf. Fig. 6.6]. The main trends observed in this series [Fig. 6.2(e)] are evident by the comparison of two exemplary spectrograms at $-20 \mathrm{~V}$ and $-100 \mathrm{~V}$ bias [Figs. 6.2(a) and 6.2(b), respectively]. We focus our discussion on three characteristic features, which are the maximum energy $E_{\max }$ at $t_{0}=0.4 \mathrm{ps}$, the minimum energy $E_{\text {min }}$ near the emission phase of $t_{0} \approx 0.2 \mathrm{ps}$, and the energy level $E_{\text {on }}$ at the "onset" of the photocurrent at $t_{0} \approx 0.2$ ps after the interval of suppressed photocurrent, as marked in Fig. 6.2(d). We will use these features to quantitatively determine the strength and nanoscopic spatial profile of the electric field surrounding the tip.

The most prominent differences between the streaking spectrograms at $-20 \mathrm{~V}$ and $-100 \mathrm{~V}$ bias appear at $E_{\min }$ and $E_{\text {on }}$. With increasing negative bias voltage, both 
energies decrease relative to the contribution of the bias voltage, $-e U_{\text {bias }}$ (dashed line in Figs. 6.2(a)-(d)). Whereas the minimum kinetic energy essentially coincides with the bias energy at $U_{\text {bias }}=-20 \mathrm{~V}$ [cf. Fig. 6.2(a)], it drops by more than $10 \mathrm{eV}$ below the bias energy level for $U_{\text {bias }}=-100 \mathrm{~V}$ [cf. Fig. 6.2(b)].

The bias-dependencies of $E_{\max }, E_{\min }$, and $E_{\text {on }}$ are displayed in Fig. 6.2(f), extracted from the series of measurements in Fig. 6.2(e), and offset by the bias energy. The maximum THz-induced energy gain $\left(E_{\max }+e U_{\text {bias }}\right)$ is nearly constant at about $26 \mathrm{eV}$ for all bias voltages (black squares). In contrast, both the minimum and the onset energies (blue and red squares, respectively) depend linearly on bias voltage with a common slope of $0.135 \mathrm{eV} / \mathrm{V} \cdot U_{\text {bias }}$, such that $E_{\text {min }}+e U_{\text {bias }}=0.135 \mathrm{eV} / \mathrm{V} \cdot U_{\text {bias }}$ and $E_{\text {on }}+e U_{\text {bias }}=19 \mathrm{eV}+0.135 \mathrm{eV} / \mathrm{V} \cdot U_{\text {bias }}$.

\subsection{Numerical simulation of streaking spectrograms}

Obtaining a quantitative understanding of nanostructure streaking in the presence of static fields requires a detailed consideration of the joint action of the superimposed static and dynamic fields on the photoelectrons. The final kinetic energy $E_{\text {end }}$ of a photoelectron with initial kinetic energy $E_{0}$ is given by the integral over the electric field along the trajectories $s(t)$ corresponding to the time-dependent distance from the tip surface:

$$
E_{\text {end }}=E_{0}-e \int_{0}^{\infty}\left[F_{\text {stat }}(s)+F_{T H z}(s(t), t)\right] d s
$$

The electric field at the nanotip apex is the superposition of the static field $F_{\text {stat }}(s)$ and the time-dependent $\mathrm{THz}$ near-field $F_{T H z}(s(t), t)$. The THz-induced energy gain, i.e., the second term in the integral, depends on the individual trajectories $s(t)$. Besides the initial kinetic energy $E_{0}$ and the emission time $t_{0}$, these trajectories depend on the bias voltage $U_{\text {bias }}$. In particular, the strength of the static field driving the electrons away from the tip determines their effective interaction time with the $\mathrm{THz}$ near-field. Predicting the quantitative influence of the bias field on the streaking process, however, warrants numerical modeling.

In our simulations of streaking spectrograms, we describe the $\mathrm{THz}$ field by its field strength $F_{T H z}^{0}$ at the tip surface $(s=0)$ and a dipolar spatial decay length $l_{f}$ (see Appendix 6.9). The local $\mathrm{THz}$ waveform is determined from experimental data (see Appendix 6.9). The static electric field is modeled as a superposition of a homogeneous long-range component with field strength $F_{\text {stat }}^{1}$ and a more confined, tip-induced component with a surface field of $F_{\text {stat }}^{0}$ and decay length $l_{f}$ also used for the THz near-field. The variables for the static field are constrained to satisfy the total energy $-e U_{\text {bias }}$ gained by the bias voltage [first term in the integral in Eq. 6.1].

In analogy to the widely-used simpleman's model of strong-field photoemission from 
(a)

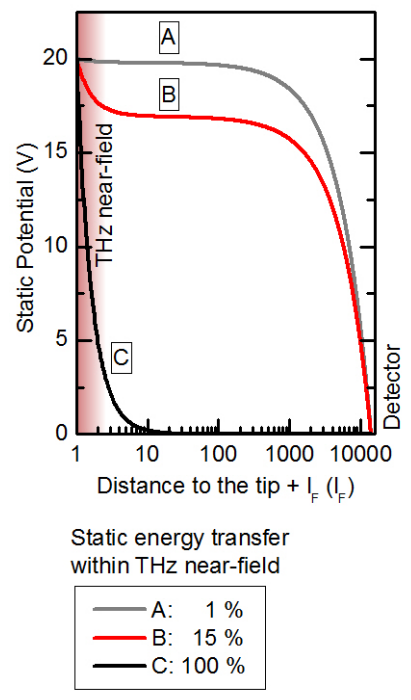

(b)

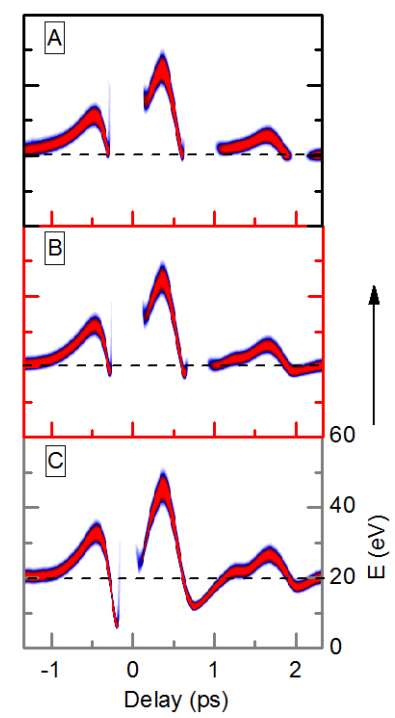

Figure 6.3: Influence of the static bias onto the streaking spectrograms. (a) Spatial decay of the static potential for three scenarios. The red-shaded area represents the $\mathrm{THz}$ near-field. A: $1 \%$-fraction of $-e U_{\text {bias }}$ is gained on the scale of the $\mathrm{THz}$ near-field. B: $15 \%$-fraction C: At $100 \%$, the electron is accelerated to its final velocity within the $\mathrm{THz}$ near-field. (b) Simulated streaking spectrograms for cases A-C.

atoms $[128,129]$ or metallic nanotips $[16,115]$, the simulation of the spectrograms is composed of two steps, namely (i) the photoemission process and (ii) the field-driven propagation of photoelectrons. The photoemission is described as a multiphoton process using the Fowler-Dubridge model [34,35] and includes the reduction of the effective work function via the Schottky effect [29]. For each delay, the field-dependent initial electron energy spectrum at the surface is taken into account prior to the propagation in the $\mathrm{THz}$ near-field. The propagation step computes point-particle electron trajectories in the spatio-temporally varying field composed of the static and THz-frequency components (see Appendix 6.9).

From the simulations, we generally find that the delay-dependent photocurrent modulation and the energy distribution are governed by both the field-dependence of the emission probability and propagation effects. Whereas the enhancement of the photocurrent is an instantaneous effect due to Schottky barrier-reduction by the static and $\mathrm{THz}$ fields, the suppression of the photocurrent results from the propagation of photoelectrons back to the tip.

\subsection{Influence of the near-field parameters on the electron dy- namics}

The streaking measurements contain direct information on the spatial decay of the static and $\mathrm{THz}$ fields close to the nanotip apex (see Appendix 6.9). For example, the energies $E_{\text {min }}$ and $E_{\text {on }}$ measured in the streaking spectrograms reflect the degree, to which the static potential drops within the confined region of the $\mathrm{THz}$ near-field. Figure 6.3 illustrates the change in the computed streaking spectrograms for three scenarios in the spatial profile of the bias field. These cases correspond to an essentially homogeneous static field (A, no lightning-rod field enhancement), a fully localized static field with a 
sharp drop over the distance $l_{f}(\mathrm{C})$, or a mixed scenario (B).

If the electrons acquire the bias energy essentially outside the near-field region (A), the streaking waveform is merely shifted by the bias and is restricted to the accelerating half-cycles of the $\mathrm{THz}$ transient, and $E_{\text {min }} \approx-e U_{\text {bias }}$. The situation becomes more complex if there is a considerable decay of the bias field within the $\mathrm{THz}$ near-field region, illustrated in $(\mathrm{B}, \mathrm{C})$. Here, the minimum energy is influenced by the temporal evolution of the $\mathrm{THz}$ transient, and one finds kinetic energies below the bias energy, i.e., $E_{\text {min }}<-e U_{\text {bias }}$.

Let us now relate these simulated trends to the experimental observations. At low bias voltages [cf. Fig. 6.2(a), -20 V], the minimum kinetic energy in the streaking spectrograms largely coincides with the bias energy level. With increasing acceleration in the negative bias field, the minimum energy decreases relative to the bias energy level by about $0.135 \mathrm{eV}$ per Volt applied negative bias [cf. Fig. 6.2(f)]. To account for this scaling, we simulated streaking traces in a static potential partially decaying within the THz near-field [cf. Figs. 6.3(B), 6.2(c) and 6.2(d)]. The series of measurements displayed in this work is best reproduced by a minimum surface $\mathrm{THz}$ field strength of $F_{T H z}^{0}=-3.15 \mathrm{MV} / \mathrm{cm}$ and a THz decay length of $l_{f}=55 \mathrm{~nm}$. Considering the incident $\mathrm{THz}$ field strengths of $40 \mathrm{kV} / \mathrm{cm}$, we estimate a field enhancement factor about 80 . The static energy gain within the $\mathrm{THz}$ near-field amounts to $15.7 \mathrm{eV}$ for $-100 \mathrm{~V}$ bias voltage, corresponding to a static surface field of $F_{\text {stat }}^{0}=-1.45 \mathrm{MV} / \mathrm{cm}$. This energy gain is relatively independent of the specific functional form of the bias field.

Figures $6.2(\mathrm{c})$ and $6.2(\mathrm{~d})$ show simulated streaking spectrograms for a low $(-20 \mathrm{~V})$ and a high $(-100 \mathrm{~V})$ negative bias voltage, respectively, for direct comparison to the experimental measurements in Figs. 6.2(a) and 6.2(b). We simulate the experimental streaking spectrograms with one universal set of parameters for all bias voltages. Overall, these simulations are in remarkable agreement with the measured spectrograms. Specifically, our simulations reproduce the experimentally observed scaling of the characteristic energies $E_{\max }, E_{o n}$, and $E_{\min }$, as shown in Fig. 6.2(f). The energy gain in the $\mathrm{THz}$ field at $E_{\max }$ is independent of bias voltage, which is a clear sign of field-driven dynamics. The linear decrease of the energies $E_{\text {on }}$ and $E_{\text {min }}$ results from the decay of the static potential within the $\mathrm{THz}$ near-field (specifically, $15 \%$ of the static potential decays within $l_{f}$ ). The full set of spectrograms is provided in the Appendix, and a movie of experimental and simulated spectrograms can be found in the Supplementary Online Material [130].

\subsection{Caustic trajectories}

The numerical simulations reproduce fine details of the experimental spectrograms, and allow for an in-depth analysis of the underlying computed electron dynamics and trajectories. For example, we will discuss the minimum energy feature prior to the gap 

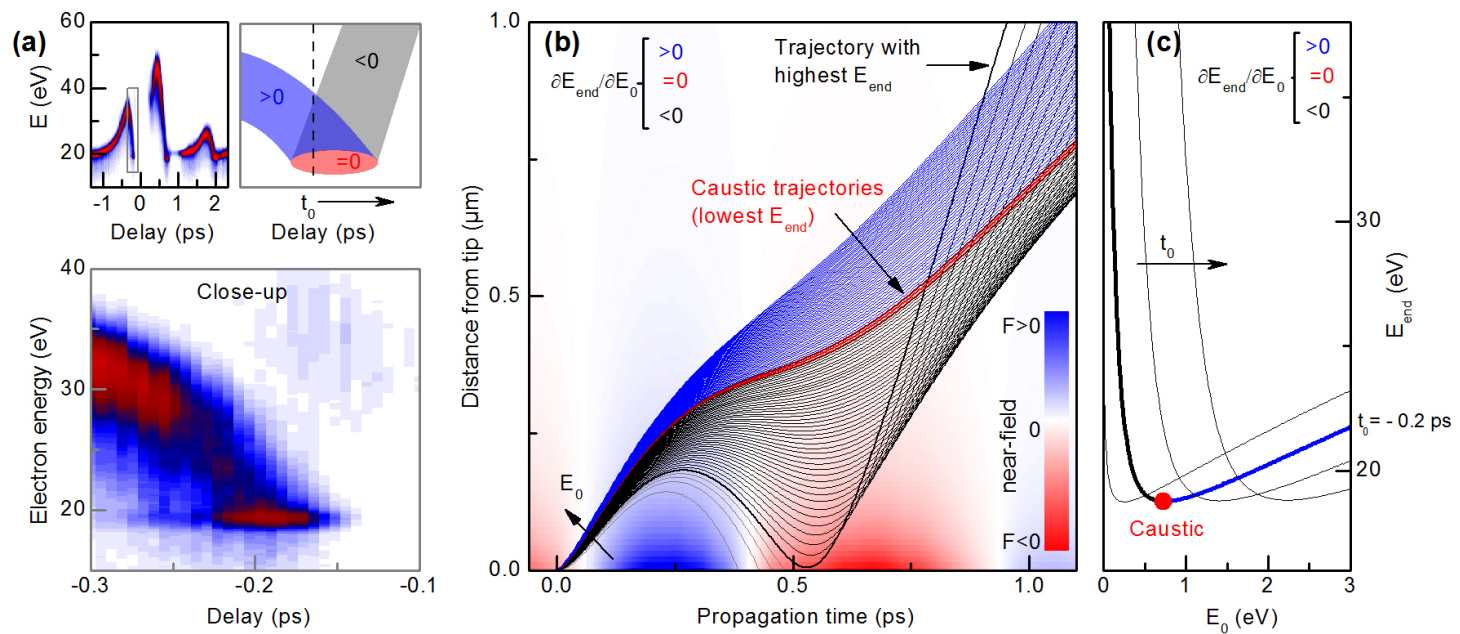

Figure 6.4: Caustic trajectories at $-20 \mathrm{~V}$ bias voltage. (a) Experimental streaking trace with a close-up at emission times directly before a complete suppression of the photocurrent. (b) Simulated trajectories with different initial energies. The background colors show the time-dependent near-field. The colors of the trajectories refer to the respective sections in (c). (c) Final energy as a function of initial energy (transfer function) featuring a minimum in the final energy (caustic, red). Emission for time $t_{0}$ and additional curves for delays in steps of $10 \mathrm{fs}$.

around $t_{0}=-0.2$ ps [see Fig. 6.4(a)]. The characteristics of this spectral feature are a high electron yield at the energy minimum and a smaller number of electrons with higher energy. The minimum energy is rather well-defined and nearly constant over a delay interval of around $100 \mathrm{fs}$.

This spectral feature implies a considerable THz-induced spectral reshaping of the initial photoelectron spectrum [15]. We investigate this characteristic detail by simulating electron trajectories for a distribution of initial kinetic energies, as shown in Fig. 6.4(b). The final kinetic energy of the photoelectrons as a function of their initial energy is presented in Fig. 6.4(c) for different emission times. This plot depicts the energy transfer function relating the final energy $E_{\text {end }}$ to the initial energy $E_{0}$. The slope of $E_{\text {end }}\left(E_{0}\right)$ characterizes three different classes of trajectories, which are marked by the color-code in Fig. 6.4(a). In case of high initial energies (marked in blue), $\partial E_{\text {end }} / \partial E_{0}$ is positive and asymptotically approaches unity, indicating quasi-static electron acceleration with the THz-induced energy gain being independent of the initial energy. As illustrated in Fig. 6.4(b), the electrons with the lowest initial energy are too slow to escape the positive decelerating $\mathrm{THz}$ near-field (grey trajectories), which leads to a reduction of the total photocurrent. A minimum initial energy is required to escape the near-field. This critical initial energy corresponds to a grazing trajectory and the maximum final energy (thick black line). Upon further increasing the initial energy, the reacceleration in the negative half-cycle occurs at larger distances from the tip. Hence, higher initial energies lead to lower final energies, and these trajectories (black lines) exhibit a negative slope of the transfer function, $\partial E_{\text {end }} / \partial E_{0}<0$. 


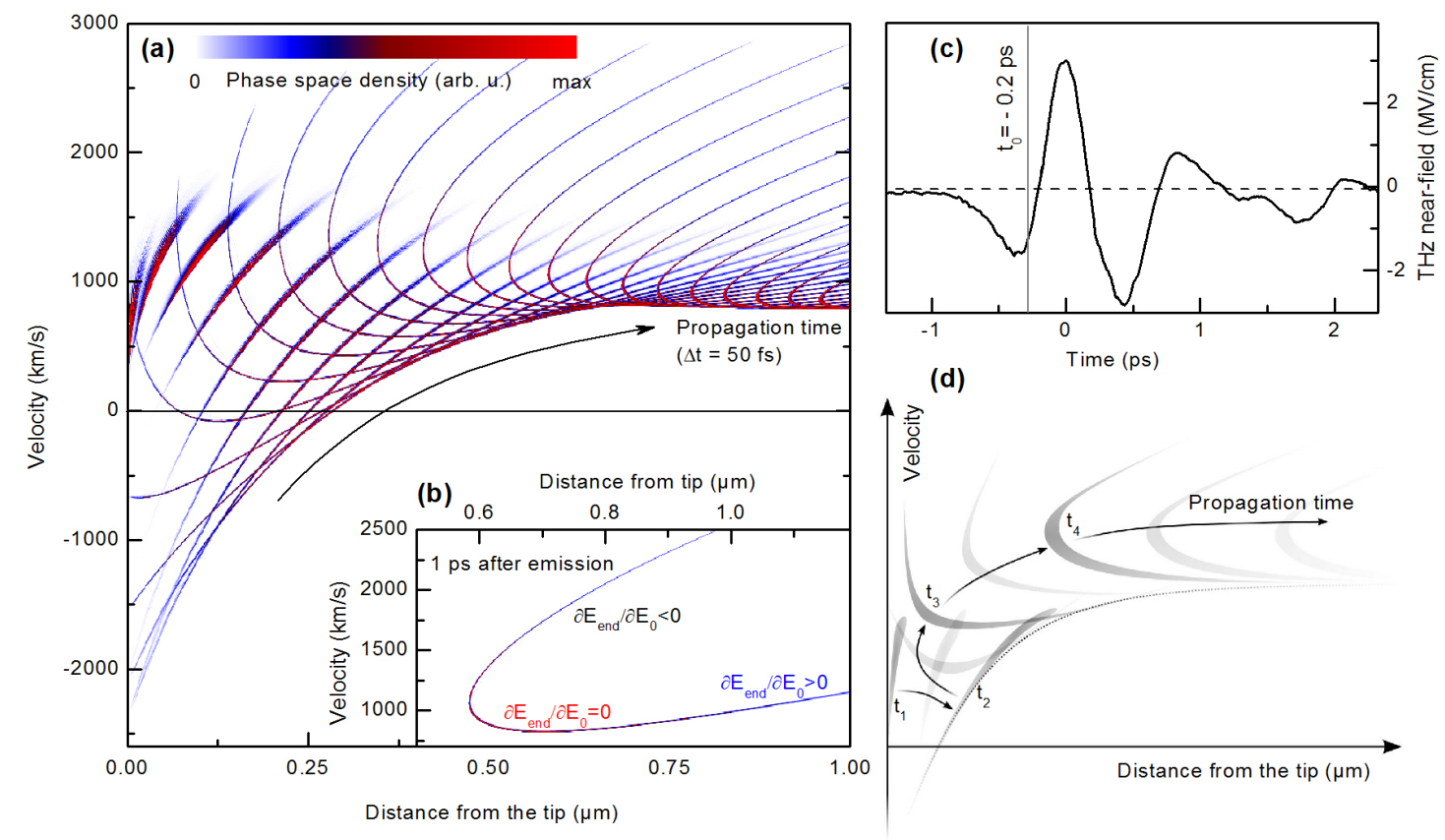

Figure 6.5: Simulated caustic trajectories in phase space (same emission time $t_{0}=-0.2 \mathrm{ps}$ and bias voltage $-20 \mathrm{~V}$ as in Fig. 6.3). (a) Phase space density distribution for different propagation times after photoemission (step width $\Delta t=50 \mathrm{fs}$, weighted with the incident spectral distribution and averaged over emission times with an FWHM of $30 \mathrm{fs}$ ). (b) Exemplary phase space density distribution $1 \mathrm{ps}$ after emission. The caustic trajectories show the lowest kinetic energy. (c) The near-field at the tip surface with the emission time marked in grey. (d) Sketch illustrating the temporal evolution of the phase space density.

At the transition between both classes of trajectories (blue and black), the transfer function changes sign (red) and its slope approaches zero. In particular, this minimum of the transfer function $E_{\text {end }}\left(E_{0}\right)$ leads to the observed caustic feature in the spectrogram, which is defined by the mapping of different trajectories onto the same final energy $\left(\partial E_{\text {end }} / \partial E_{0}=0\right)$. Notably, the respective initial energies of the caustic energy minimum shift with the delay from $E_{0}=0$ to higher initial energies, as shown in Fig. 6.4(c). Thus, the range of delays with caustic behavior is closely related to the width of the initial spectrum.

More generally, caustic trajectories occur for an appropriate combination of a rapid spatial field decay and a fast temporal rise in the accelerating field. Such a situation is also found at the onset at the second accelerating half-cycle at $t_{0}=0.2 \mathrm{ps}$; see Appendix, Fig. 6.9.

\subsection{Evolution in phase space}

Trajectories and transfer functions (cf. Fig. 6.4) provide a mapping of initial to final kinetic energies. However, to numerically evaluate the spectral reshaping and the longitudinal electron pulse properties, we incorporate the initial energy spectra and analyze the resulting time-dependent electron distribution in phase space. 
The temporal evolution of the caustic trajectories at $t_{0}=-0.2 \mathrm{ps}$ (cf. Fig. 6.4) is depicted in phase space in Fig. 6.5 (movies in Supplementary Online Material [130]). Figure 6.5(a) displays snapshots ( $\Delta t=50 \mathrm{fs}$ ) of the phase space density. Each snapshot is a scatter plot of space and velocity coordinates, extracted from the trajectories at a specific propagation time. The individual data points are weighted with the electron yield for the initial energy of the respective trajectory (further details given in Appendix 6.10). The initial electron distribution is first stretched and then warped close to the tip surface. After leaving the $\mathrm{THz}$ near-field, the underlying convex transfer function is reflected in the curved phase space density distribution (cf. Fig. 6.4c) as depicted in Fig. 6.5(b): The lower-velocity branch corresponds to the initially faster electrons with $\partial E_{\text {end }} / \partial E_{0}>0$ [blue trajectories in Fig. 6.4(b)], and the higher-velocity branch is associated with the black trajectories in Fig. 6.4(b). In Figure 6.5(b), the caustic electrons exhibit the lowest final momentum.

Generally, the manipulation of the phase space density distribution sensitively depends on the emission time $t_{0}$ into the $\mathrm{THz}$ field. Electrons emitted during increasing acceleration, e.g., at a delay of $t_{0}=0.2 \mathrm{ps}$, experience a spectral compression, as well as an overall contraction in phase space in comparison to static acceleration (cf. Fig. 6.9, Appendix 6.10).

The modification of the populated longitudinal phase space volume is enabled by the spatio-temporal variation of the $\mathrm{THz}$ near-field leading to a trajectory-dependent energy gain $E_{T H z}$. This contrasts to conservative forces, which necessarily preserve the populated phase space volume (Liouville's theorem).

\subsection{Conclusions}

In summary, we found that an external bias voltage is a powerful control parameter in streaking spectroscopy from contacted metallic nanostructures, which impacts ultrafast electron dynamics at the nanoscale. Both the waveform and the spatial profile of $\mathrm{THz}$ streaking fields can be extracted from comparing numerical simulations with characteristic features in experimental spectrograms. Caustic trajectories, which are associated with nearly grazing return trajectories at the surface, are found to be particularly sensitive to the $\mathrm{THz}$ and static field distribution. The spatio-temporal electron dynamics in $\mathrm{THz}$ near-fields allow for a tuning of electron energy and phase space distributions. Our work highlights emerging fundamental prospects for ultrafast electron pulse control arising from the combined action of static and time-dependent near-fields.

\subsection{Appendix A: Experimental methods and data analysis}

An extended series of experimental streaking spectrograms is presented for additional bias voltages in Fig. 6.6. Each spectrogram is normalized to the maximum electron 


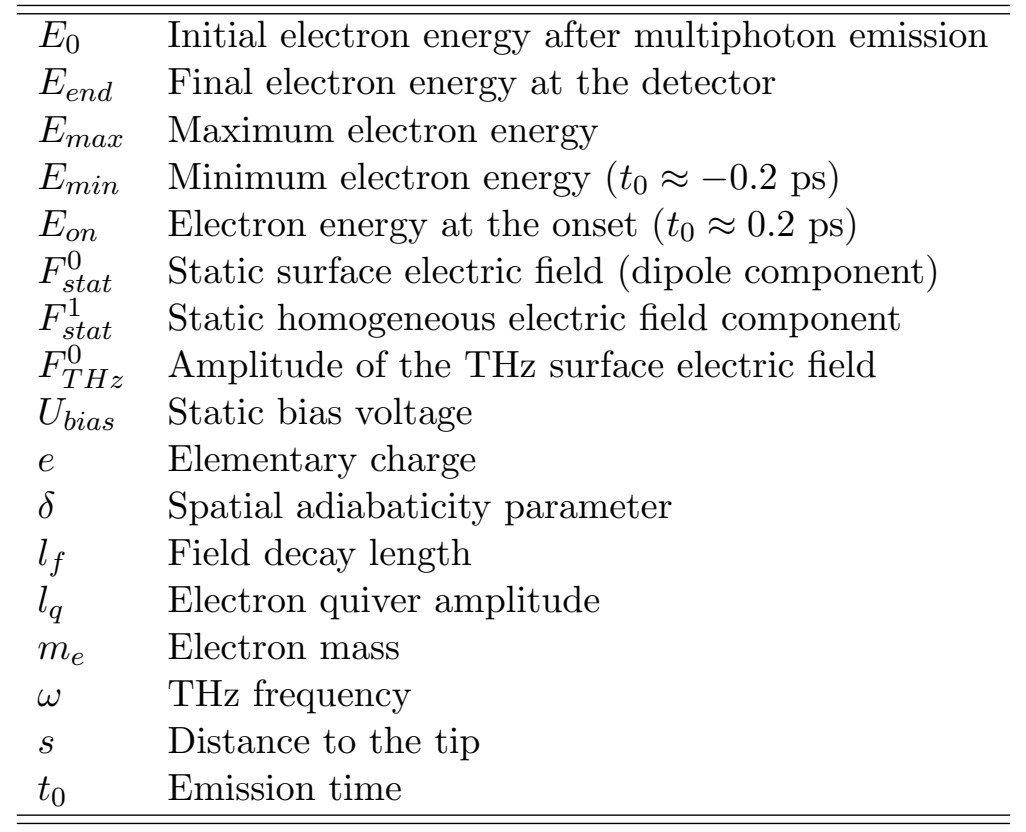

Table 6.1: Notations and abbreviations.

spectral density at large negative delay. For comparison, all streaking spectrograms use a common color scale.

The kinetic energy distributions of the photoelectrons are detected with a time-of-flight electron spectrometer. The energy resolution is given by the temporal resolution of the acquisition electronics, and varies with the kinetic energy of the photoelectrons. In order to maintain constant energy resolution for different bias potentials, we apply a negative drift voltage of $U_{\text {drift }}=U_{\text {bias }}+30 \mathrm{~V}$.

\subsection{Appendix B: Numerical simulations and analytical streak- ing model}

In this section, we describe the numerical simulation of the spectrograms in detail, and we introduce an analytical description of the electron dynamics observed in our experiments. Both the numerical and the analytical model are based on the same spatial $\mathrm{THz}$ near-field profile presented in the following. For the numerical simulations, we focus on the two-step-model of photoemission and propagation of electrons and elucidate our procedure to extract the near-field parameters from experimental data.

\subsubsection{Spatial and temporal dependence of the near-field}

As discussed in the main text, the electric field surrounding the nanotip apex is modeled consisting of two components: a static field induced by the negative bias voltage and the THz-induced near-field, which varies in time. Both components decay on the scale 


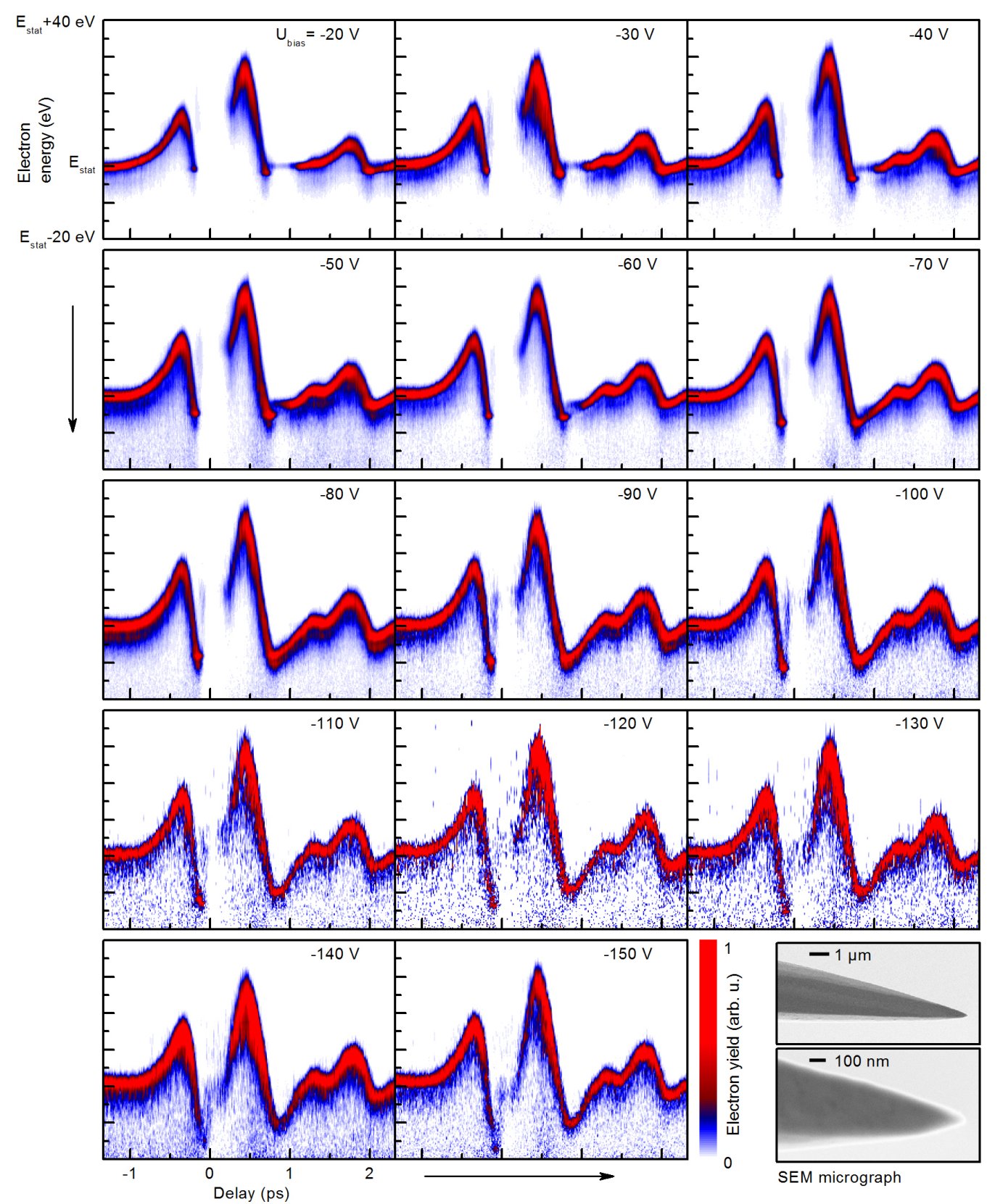

Figure 6.6: Complete series of experimental streaking spectrograms for bias voltages from -20 to $-150 \mathrm{~V}$ and SEM micrographs of the gold nanotip used to record the streaking series. 

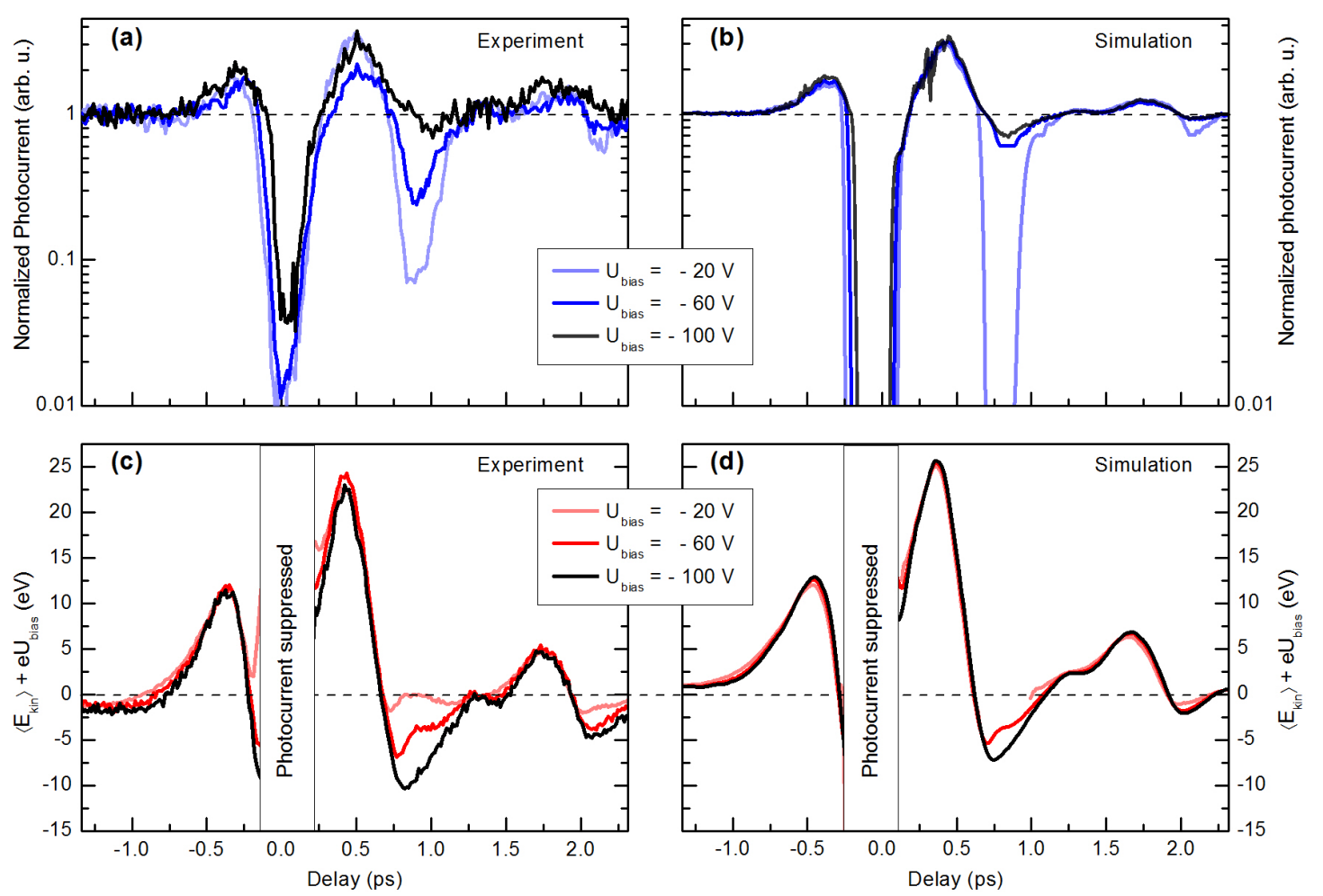

Figure 6.7: Influence of a static bias voltage onto photocurrent and kinetic energy. (a) and (b) Photocurrent, measured (a) and simulated (b) for bias voltages of $-20 \mathrm{~V},-60 \mathrm{~V}$, and $-100 \mathrm{~V}$. (c) and (d): Measured and simulated energy expectation values for same bias voltages. During the intervals of suppressed photocurrent, the assignment of kinetic energies is not possible.

of the apex curvature.

We describe the spatial dependence of the THz-induced near-field $F_{T H z}(s, t)$ by a dipolar field, characterized by a decay length $l_{f}$, defined by $F_{T H z}\left(l_{f}\right)=F_{T H z} / 2$. For convenience, we introduce a rescaled parameter $d, l_{f}=(\sqrt[3]{2}-1) \cdot d$ :

$$
F_{T H z}(s, t)=F_{T H z}^{0}(t) d^{3} /(d+s)^{3} .
$$

To construct the local $\mathrm{THz}$ near-field field $F_{T H z}^{0}(t)$, we employ a combination of the energy expectation value at a bias voltage of $-100 \mathrm{~V}$ and the incident electric field measured by electro-optic sampling (EOS). The expectation value at high negative bias represents a reliable measure of the momentary field at all times outside the suppression of the photocurrent in one half-cycle. Figure 6.8(e) compares the energy expectation value with the incident electric field, demonstrating some ringing of the near-field after the initial cycle (compare also Ref. [15]). However, this frequency-dependent response has a weaker impact on the main cycle and the half-cycle not captured by the energy expectation value. We use this observation to complete the near-field in the missing half-cycle by replacing it with a scaled half-cycle determined by EOS. 
A small delay difference of $80 \mathrm{fs}$ between the maximum of the photocurrent (corresponding to the maximum of the near-field strength) and the maximum energy of the streaking waveform is caused by the propagation in the $\mathrm{THz}$ near-field. This time difference is well-reproduced in our numerical simulations.

In our model, the static field is composed of the two spatial components, such that the total static field $F_{\text {stat }}(\mathrm{s})$ is given by:

$$
F_{\text {stat }}(s)=F_{\text {stat }}^{0} d^{3} /(d+s)^{3}+F_{\text {stat }}^{1}
$$

As introduced in the main text, $F_{\text {stat }}^{1}$ is the long-range component of the bias field ${ }^{1}$, and $F_{\text {stat }}^{0}$ is the electric field strength of the dipolar component at the surface.

\subsubsection{Photoemission process}

The NIR-induced photoemission is described as a multi-photon process following the Fowler-DuBridge theory [34-38]. It is based on the Sommerfeld model of a free electron gas and one-dimensional emission. The energy difference between the sum of n-photon energies and the initial state in the metal determines the excess kinetic energy of the emitted electrons. The relative contributions of the different emission channels are determined using the spectrally integrated photocurrent trace at $U_{\text {bias }}=-100 \mathrm{~V}$. To include the Schottky effect, we employ an effective work function [29] $\Phi_{\text {eff }}=\Phi-$ $\sqrt{\frac{e^{2} F}{4 \pi \varepsilon_{0}}}$. The model qualitatively reproduces the THz-induced photocurrent modulations for all bias voltages (cf. Fig. 6.7). Considering the overall spectral resolution in the experiments, we do not include a more detailed description of the photoemission process or carrier relaxation at the surface.

\subsubsection{Propagation of photoelectrons}

The electron trajectories are computed in a spatially and temporally varying one-dimensional electric field using a Runge-Kutta scheme for various delays between NIR and THz pulses, and are weighted by the respective initial energy spectra. This yields the final electron spectra of the streaking spectrograms.

For better comparison between measured and simulated streaking spectrograms, we convolve the numerical results with the energy resolution of the electron spectrometer.

\footnotetext{
${ }^{1}$ In the original publication, the homogeneous component is missing in the equation.
} 


\subsubsection{Extraction of the near-field parameters}

The near-field parameters are obtained via best agreement with the experimental streaking spectrograms. The maximum energy gain in the $\mathrm{THz}$ field $E_{\text {max }}+e U_{\text {bias }}$ is deter-

mined by $F_{T H z}^{0}$, and $l_{f}$, and is independent of the bias voltage $U_{\text {bias }}$, which indicates field-driven acceleration of photoelectrons.

This set of optimal parameter values allows us to reproduce the photocurrent modulation, as well as the kinetic energy trace as shown in Fig. 6.7. The photocurrent [cf. Fig. 6.7(a) and 6.7(b)] is essentially in-phase with the streaking waveform [cf. Fig. 6.7(c) and 6.7(d)], as expected for field-driven electron dynamics [15]. The photocurrent, depicted in Fig. 6.7(a), changes from a complete suppression to a strong enhancement at maximum $\mathrm{THz}$ fields. While the photocurrent enhancement is not particularly sensitive to the bias voltage in the range studied here, the influence of the static field is strongest close to the zero-crossings of the surface electric field due to a fraction of the photoelectrons propagating back to the metal surface.

We note that the experimental streaking spectrograms could also be reproduced by a somewhat different spatial dependence of the local contribution to the static field, e.g., by an exponential decay. Physically, the static energy gain of the electrons within the $\mathrm{THz}$ near-field governs the propagation effects and is thus the universal property identified. However, the general parameters obtained are very similar for different functional forms. As an illustration of the uniqueness of the parameters obtained, we fit the simulations with respect to the minimum and maximum kinetic energy $E_{\min }$ and $E_{\max }$ alone, which allows for different spatial decay lengths (cf. Fig. 6.8). For a low surface field strength and a long spatial field decay, the interaction time between the $\mathrm{THz}$ electric field and the photoelectrons increases, shifting the photocurrent onset to higher energies. Thus, only one set of parameters matches to the measurement in all three energies $E_{\text {max }}, E_{\text {min }}$, and $E_{\text {on }}$.

\subsubsection{Analytical description}

The following analytical model facilitates an intuitive physical understanding of the electron dynamics. An analytical solution for the trajectories in the spatio-temporally varying field is not directly possible; we therefore introduce several approximations: The influence of the bias onto the streaking spectrograms is described by a set of analytical equations for the three energies $E_{\max }, E_{\min }$, and $E_{\text {on }}$. We use a spatial decay of the static and the $\mathrm{THz}$ electric field analogous to our numerical model. The temporal oscillation of the driving field is given by a $\cos (\omega t)$ term $^{2}$ :

$$
E_{\text {end }}=e \int_{0}^{d e t} F_{T H z}^{0} \cos (\omega t) \frac{d^{3}}{(d+s)^{3}}-F_{\text {stat }}^{0} \frac{d^{3}}{(d+s)^{3}}-F_{\text {stat }}^{1} .
$$

\footnotetext{
${ }^{2}$ In the original publication, the cosine is missing in the equation.
} 

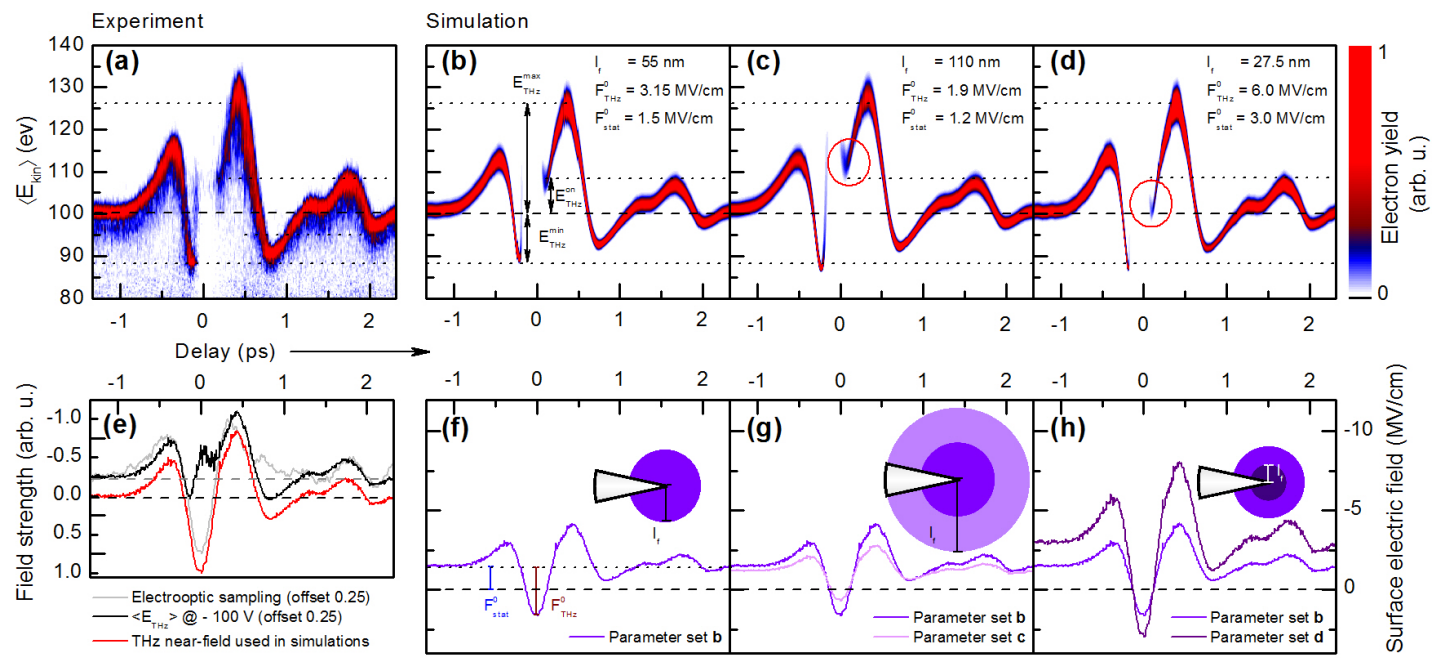

Figure 6.8: Influence of the near-field parameters on the streaking spectrograms. (a) Measured spectrogram at $-100 \mathrm{~V}$ bias. (b) Simulated spectrogram with optimized parameters. (c) Simulated spectrogram with larger decay length of the $\mathrm{THz}$ field $\left(l_{f}\right.$ doubled). $F_{T H z}^{0}$ and $F_{\text {stat }}^{0}$ are used to adjust maximum and minimum kinetic energies. The resulting "onset" energy $E_{\text {on }}$ of the photocurrent at $t_{0}=0.2 \mathrm{ps}$ (cf. red circle) is too high. (d) Simulated spectrogram with a very rapidly decaying $\mathrm{THz}$ field $\left(l_{f}\right)$. Here, the onset energy $E_{\text {on }}$ is too low (cf. red circle). (e) The incident electric field used in the simulations is extracted from the energy expectation value and electro-optic sampling. (f) Temporal evolution of the total surface electric field applied in simulation (b). The sketch illustrates the spatial decay of the electric field. (g) and (h): Analogous illustration for the simulations shown in (c) and (d), respectively.

As discussed earlier, electrons at the maximum energy of the streaking trace $\left(t_{0}=\pi / \omega\right)$ exhibit quasi-static field-driven dynamics, and the time-dependence of the electric field can be neglected in computing the final energy. We obtain for the maximum energy gain in the $\mathrm{THz}$ field:

$$
E_{\text {max }}+e U_{\text {bias }}=\frac{e F_{T H z}^{0} d}{2}
$$

At the onset of the photocurrent at $t_{0} \approx \pi / 2 \omega\left(t_{0} \approx 0.2 \mathrm{ps}\right.$ in the experiment), the time dependence of the electric field is approximated by a Taylor expansion of first order, and we obtain the THz-induced onset energy component:

$$
E_{\text {on }}+e U_{\text {bias }}=-e \int_{0}^{\infty}\left(F_{T H z}^{0} \omega \tau-F_{\text {stat }}^{0}\right) \frac{d^{3}}{(d+s)^{3}} d s .
$$

Via the definition of the onset, the total surface electric field in the moment of emission is zero $\left[0=F_{\text {stat }}^{0}+F_{T H z}^{0}\left(t_{0}\right)\right]$. As the respective energy is evaluated for the THz near-field, the contribution due to acceleration in the homogeneous static field $F_{\text {stat }}^{1}$ is neglected. The integral becomes: 


$$
E_{\text {on }}+e U_{\text {bias }}=-\frac{e d}{2}\left(F_{T H z}^{0} \omega \tau-F_{\text {stat }}^{0}\right)
$$

An increased static field shifts the emission time at the onset to phases of negative $\mathrm{THz}$ force (positive $\mathrm{THz}$ field strength). As the temporal slope of the $\mathrm{THz}$ transient at the surface is constant, the propagation time $\tau$ in the near-field and, thus, the THz-induced energy gain also remains constant $(\approx 19 \mathrm{eV})$. Thus, the external bias does not change the trajectories at the onset. The final kinetic energy of the photoelectrons results from this near-field energy component $(\approx 19 \mathrm{eV})$ and the the static long-range component. The observed decrease of the THz energy gain $E_{\text {on }}+e U_{\text {bias }}\left(0.135 \mathrm{eV} / \mathrm{V} \cdot U_{\text {stat }}\right)$ at the onset arises from a decreased $\mathrm{THz}$-acceleration and corresponds to the energy transferred by the rapidly decaying static field component.

The same linear dependence of this onset energy (at $t_{0}=0.2 \mathrm{ps)} \mathrm{is} \mathrm{observed} \mathrm{at} \mathrm{the}$ energy minimum for $t_{0}=-0.2$ ps (i.e. $t_{0} \approx \pi / 2 \omega$ ): The kinetic energy directly after the escape from the $\mathrm{THz}$ near-field amounts to nearly zero, leading to the following equation for the energy minimum:

$$
E_{\text {min }}+e U_{\text {bias }}=\frac{e F_{\text {stat }}^{0} d}{2}
$$

In this approximation, the final energy results from the spatial integral over the homogeneous component of the static field alone. This value thus represents the lower limit of the minimum electron energy. Remarkably, the deviation between the numerical simulation and the analytical approximation is less than $2 \mathrm{eV}$.

At this point, the three equations 6.5, 6.7, and 6.8 describe three essentiall energies, which yield the near-field parameters from experimentally found energies:

- Maximum:

$E_{\text {max }}+e U_{\text {bias }}=\frac{e F_{T H z}^{0} d}{2}=26 \mathrm{eV}$

- Onset:

$$
E_{\text {on }}+e U_{\text {bias }}=-\frac{e d}{2}\left(F_{T H z}^{0} \omega \tau-F_{\text {stat }}^{0}\right)=19 \mathrm{eV}+0.135 \mathrm{eV} / \mathrm{V} \cdot U_{\text {bias }}
$$

- Minimum:

$$
E_{\text {min }}+e U_{\text {bias }}=\frac{e F_{\text {stat }}^{0} d}{2}=0.135 \mathrm{eV} / \mathrm{V} \cdot U_{\text {bias }}
$$

- Static contribution:

$$
E_{\text {stat }}=-e\left(\frac{F_{\text {stat }}^{0} d}{2}+F_{\text {stat }}^{1} d_{\text {det }}\right)=-e U_{\text {bias }}
$$

$d_{\text {det }}=3 \mathrm{~mm}$ is the distance from the tip to the detector. Solving this system of equations for a center frequency of $\omega=2 \pi \cdot 1.2 \mathrm{THz}$ leads to $F_{\text {stat }}^{1}=290 \mathrm{~V} / \mathrm{cm}, F_{\text {stat }}^{0} / F_{\text {THz }}^{0}=0.51$ and $\tau=96$ fs. This value relates to the experimentally observed temporal shift of $80 \mathrm{fs}$ between the maximum of the photocurrent and the kinetic energy trace.

Whereas the system of equations describes our experimentally observed bias-impact onto 


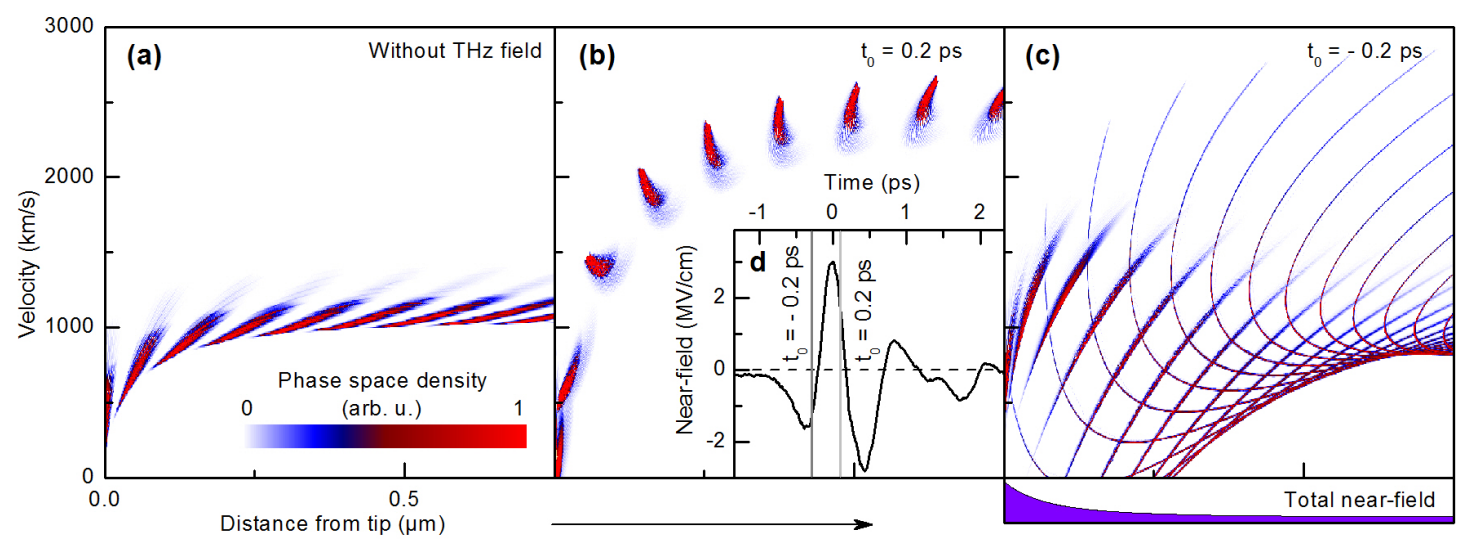

Figure 6.9: Time evolution of the phase space density distribution simulated for different emission times at $-20 \mathrm{~V}$ bias voltage. (a) Evolution of the phase space distribution of an electron pulse accelerated by the static field of the bias voltage alone $(\Delta t=100 \mathrm{fs})$. (b) Phase space density for emission time $t_{0}=0.2 \mathrm{ps}$. Snapshots taken with time differences of $\Delta t=50 \mathrm{fs}$. Note the caustic flipping of the phase space distribution at velocities of $\approx 1300$ $\mathrm{km} / \mathrm{s}$. (c) Phase space density distribution for $t_{0}=-0.2 \mathrm{ps}$ with $\Delta t=50 \mathrm{fs}$ as shown in Fig. 6.5 (negative velocities cropped in the diagram). The spatial decay of the near-field (absolute value of the maximum THz field, no time dependence) is depicted below (c). (d) Surface electric field of the $\mathrm{THz}$ transient and the static bias with the emission times from (a) and (c) marked in grey.

the streaking spectra, they still do not allow for an explicit solution of the near-field parameters. However, using the extracted parameters, we can determine an upper limit for the spatial decay length $l_{f}$ based on the acceleration to $19 \mathrm{eV}$ at the photocurrent onset: $d=\tau \sqrt{2 \cdot 19 \mathrm{eV} / m_{e}} \approx 250 \mathrm{~nm}$, corresponding to a decay length of $l_{f}=65 \mathrm{~nm}$.

\subsection{Appendix C: Computation of the phase space density dis- tribution}

The phase space density distribution is calculated with trajectories around a common temporal delay and for different initial energies. From these trajectories, we extract the distance from the tip $s$ and the electron momentum $p=m_{e} v$. The elements in phase space are weighted by the initial energy spectrum. To account for the finite duration of the photoemission pulse, the NIR-induced photoemission is averaging over a Gaussianshaped emission window of 30 fs.

The phase space density distribution of the electron pulses strongly depends on the relative emission phase within the $\mathrm{THz}$ cycle. To illustrate the variety of possible electron dynamics, we analyze the time evolution of the electron pulse in phase space for three exemplary emission times, as shown in Fig. 6.9.

The phase space evolution is traced via snapshots taken at equidistant temporal delays of $\Delta t=50$ fs [cf. Figs. 6.9(b) and 6.9(c)] or $\Delta t=100$ fs [cf. Fig. 6.9(a)]. Figure 6.9(b) depicts the evolution of the phase space density distribution at the photocurrent onset $\left(t_{0}=0.2 \mathrm{ps}\right)$. Here, the acceleration by the $\mathrm{THz}$ electric field is strongly increasing in 
time, which results in a caustic partial inversion of the phase space density distribution. For comparison, Fig. 6.9(a) shows the acceleration of electron pulses by the static field in phase space. Finally, in Fig. 6.9(c), the phase space density distribution at the energy minimum is depicted.

At a distance of $s=1 \mu \mathrm{m}$ from the surface, a comparison of the pulse duration with and without THz-field shows an electron pulse duration of 24 fs and 82 fs (FWHM), respectively. This result highlights the potential of the THz near-field control for generating tailored ultrashort electron pulses. 


\title{
Chapter 7
}

\section{Clocking plasmon nanofocusing by THz near-field streaking}

\author{
L. Wimmer, B. Schröder, M. Sivis, G. Herink, and C. Ropers \\ Appl. Phys. Lett. 111, 131102 (2017) \\ DOI: $10.1063 / 1.4991860$
}

We apply THz near-field streaking in a nanofocusing geometry to investigate plasmon polariton propagation on the shaft of a conical nanotip. By evaluating the delay between a streaking spectrogram for plasmon-induced photoemission with a measurement for direct apex excitation, we obtain an average plasmon group velocity, which is in agreement with numerical simulations. Combining plasmon-induced photoemission with $\mathrm{THz}$ near-field streaking facilitates extensive control over localized photoelectron sources for time-resolved imaging and diffraction.

Propagating surface plasmon polaritons (SPPs) enable ultrafast energy transport at metal surfaces on the nanoscale [55, 131, 132], facilitating, for example, plasmonic nanocircuits [133-136], plasmonic vortices [137, 138] and nanofocusing [62, 66, 69, 139141]. Conical tapers represent the quintessential structure for adiabatic nanofocusing [69, 71, 139, 142-144], harnessing the efficient coupling of propagating SPPs to a local apex excitation, which is acompanied by a slowing of the SPP group velocity [69, 71 , 139]. The strong confinement of the plasmonic focus gives rise to various linear and nonlinear optical processes, with applications in microscopy and spectroscopy [59, 145-150]. Grating coupling was established for the excitation of SPPs on nanotapers [62], providing for a spatial separation of far-field from near-field components and supporting background-free scanning probe techniques [146, 148, 149, 151-153]. Moreover, the nanofocusing of SPPs by femtosecond laser pulses was shown to induce the emission of ultrashort electron pulses [59, 149, 150], contributing to the development of compact 

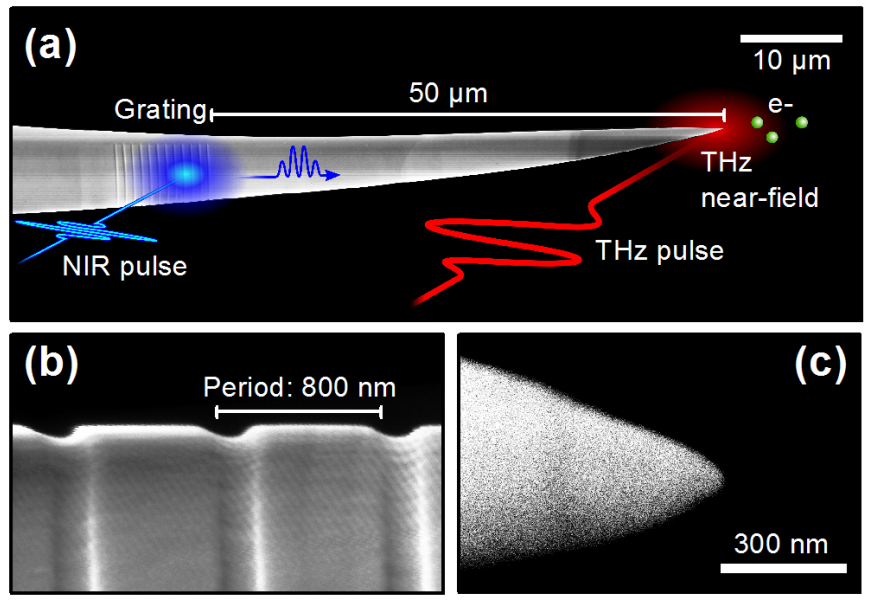

Figure 7.1: Scanning electron microscope images of the nanotip used in the experiment. (a) Micrograph of the nanotip including a sketch of the experiment. (b) Close-up of the grating structure that is optimized for incident light of $800 \mathrm{~nm}$. (c) Close-up of the tip apex (radius of curvature: $20 \mathrm{~nm}$ ).

ultrafast electron guns $[59,80,154-156]$ and time-resolved point projection microscopy $[150,157]$. Such electron sources provide a complementary approach to localized electron emission driven by direct apex illumination [16, 89, 91, 92, 94, 158-160].

For each of these approaches, a detailed characterization of the underlying plasmon dynamics is desired to address questions regarding, e.g., the optimization of the excitation process[161], the characterization of different plasmon modes [162] and the reflection of SPPs near the apex $[60,61]$. SPP propagation times and dispersion were recently investigated on thin nanorods [163-165] and plasmonic nanotapers [144] by interferometric means.

Photoelectron streaking is another powerful technique to access time-dependent electric fields. Prominently applied in attosecond science [3, 5], all-optical streaking was established for the analysis of electromagnetic transients, with further applications in the terahertz $(\mathrm{THz})$ frequency range $[6,7,15]$. Streaking spectroscopy was proposed for the phase-resolved study of spatiotemporal near-field evolutions within nanostructures [42, 44, 51-53]. Experimentally, near-field streaking at nanostructures was first demonstrated at $\mathrm{THz}$ frequencies for metal nanotips [15, 17, 43], and recently implemented using attosecond pulses $[127,166]$. Besides the use in characterizing time-dependent near-fields, the strong field enhancement and local confinement in nanostructures has enabled the all-optical control and compression of photoelectron pulses [14, 15, 43].

In this work, we apply $\mathrm{THz}$ near-field streaking to a plasmonic nanofocusing geometry and study the spatiotemporal SPP propagation on the shaft of a conical nanotip. THz streaking on plasmonic nanotapers combines the advantages of plasmon-induced photoemission, i.e., the spatial separation of excitation and emission site, with the capability to actively manipulate electron pulses. Comparing spectrograms employing SPP-mediated emission with direct apex excitation, our measurements yield the absolute propagation time from the grating to the apex.

The experimental scheme is depicted in Fig. 7.1(a), showing a scanning electron microscope (SEM) image of the tip used in the experiment, overlaid with a sketch of the plasmon and $\mathrm{THz}$ excitation. The nanotips are produced by electrochemical etching of annealed gold wires [59, 146, 148], and the grating coupler is milled into the tip shaft 

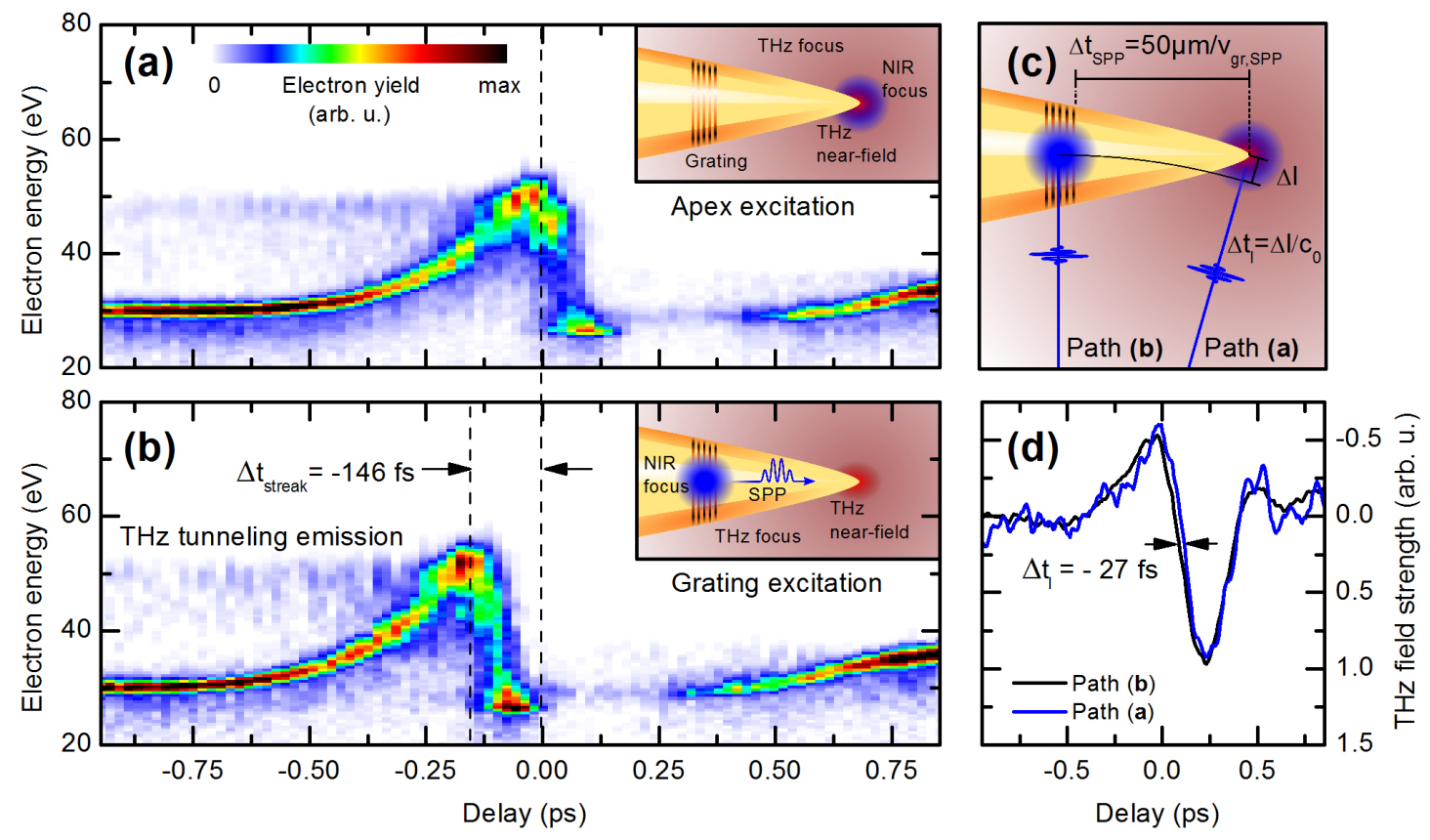

Figure 7.2: Measuring the plasmon propagation time. (a) Streaking spectrogram with the NIR beam pointing to the tip apex. (b) Streaking spectrogram with plasmon excitation at the grating coupler (see sketch). In (a), (b), the incident $\mathrm{THz}$ field strength was approx. $7 \mathrm{kV} / \mathrm{cm}$ and a static bias voltage of $U_{\text {bias }}=-30 \mathrm{~V}$ was applied to the tip. (c) Sketch: change of the optical path length from configuration (a) to (b). (d) Electrooptic sampling traces for the configurations in (a) and (b).

by a focused ion beam. Femtosecond near-infrared (NIR) pulses (duration $50 \mathrm{fs}$, center wavelength $800 \mathrm{~nm}$, repetition rate $1 \mathrm{kHz}$ ) from an amplified Ti:sapphire laser system are used for the photoelectron emission from the nanostructure and the generation of single-cycle $\mathrm{THz}$ transients. The $\mathrm{THz}$ transients are generated with the AC bias method [74] by the major part of the NIR pulse energy (1.7 mJ combined energy in the fundamental and second harmonic of the NIR pulses) in a light-induced air-plasma, which allows for a control of the incident field strength and the carrier-envelope phase. Focused by a parabolic mirror of $2.54 \mathrm{~cm}$ focal length onto the tip apex, $\mathrm{THz}$ field strengths up to $100 \mathrm{kV} / \mathrm{cm}$ can be reached in this setup. The present measurements employ incident field strengths of $7 \mathrm{kV} / \mathrm{cm}$.

A weak part of the NIR pulses (pulse energy approx. $60 \mathrm{~nJ}$ ) is focused onto a gold nanotip by a $15 \mathrm{~cm}$ lens to a focus of approx. $25 \mu \mathrm{m}$ diameter, inducing photoelectron emission either by direct apex excitation or via nanofocused SPPs created in a grating on the tip shaft [distance from the apex: $50 \mu \mathrm{m}$, cf. Fig. 7.1(b) and 7.1(c)]. The precise focus position on the tip was identified by spatial scans, as in Ref. [59]. Most efficient SPP excitation was found for illumination near the grating edge. Thus, we do not observe any retardation of the plasmon velocity due to multiple scattering within or transmission through the grating [167].

For both excitation conditions, the emitted electrons are streaked in a $\mathrm{THz}$ near-field, 
and the electron kinetic energy distributions as a function of relative THz-NIR pulse delay are acquired with a time-of-flight spectrometer. As discussed in our previous work, the resulting streaking spectrograms contain detailed information about the spatiotemporal electron dynamics in the near-field [15, 17, 43].

The recorded spectrograms are displayed in Fig. 7.2. In the graphs, increasing delays correspond to the NIR pulse arriving later at the tip apex. The spectrogram in Fig. 7.2 (a) is recorded with the NIR focus placed on the tip apex. To obtain the spectrogram of plasmon-induced photoemission [cf. Fig. 7.2(b)], the NIR beam is instead directed to the grating by tilting a mirror in about $40 \mathrm{~cm}$ distance from the focusing lens, holding the tip position constant. Both spectrograms exhibit the same overall shape and share even fine details (e.g., the enhanced feature at minimum energy and the following photocurrent suppression), which implies very similar conditions for the emission and propagation of the photoelectrons. The narrow spectral width in both direct and grating induced photoemission at negative delays implies that the emission process is multiphoton photoemission in the perturbative regime, consistent with estimates of the local field strengths. The spectrogram for grating excitation is shifted to negative delays with respect to that for apex excitation, which implies that the external NIR path length needs to be shortened in order to overlap the two pulses and access the same feature in the $\mathrm{THz}$ transient. Thus, the delay shift from Fig. 7.2(a) to Fig. 7.2(b) primarily represents the additional time required for the SPP wave packet to propagate from the grating to the apex.

We determine the delay shift between the spectrograms in Figs. 7.2(a) and 7.2(b) to $\Delta t_{\text {streak }}=-146$ fs by the maximum in their cross-correlation. The cross-correlation of the electron yield is computed separately for each energy, and the overall delay shift is found from an average of these values. To obtain the propagation time of the plasmon polaritons, we also need to take into account the change of optical path length $\Delta l$ due to the repositioning of the NIR focus from the optical grating to the apex [see Fig. 7.2(c)]. The corresponding contribution $\Delta t_{l}=\Delta l / c_{0}=-27 \mathrm{fs}$ is measured by electro-optic sampling [cf. Fig. 7.2(d)], replacing the tip with a $500 \mu \mathrm{m}$ thick ZnTe crystal. Hence, the pure SPP propagation time, more specifically the group delay time, amounts to $\Delta t_{S P P}=-\left(\Delta t_{\text {streak }}+\Delta t_{l}\right)=173 \mathrm{fs}$. For the $50 \mu \mathrm{m}$ distance between the optical grating and the tip apex, this corresponds to an average plasmon velocity of $96 \%$ of the vacuum speed of light.

We simulated the propagation of SPPs along the tip shaft using an adiabatic model, which describes the plasmon velocity as a function of the taper diameter [69, 71] (dielectric constants from Ref. [168]). The results of the simulation are summarized in Fig. 7.3. The taper radii along the tip shaft [cf. Fig. 7.3(a)] are extracted from the SEM image in Fig. 7.1(a) to compute the local group velocity (solid blue) and propagation time (solid red) of the plasmons propagating from the grating to the tip apex [Fig. 7.3(b)]. In the last few micrometers close to the apex, the decreasing taper radius influences the dispersion relation, which leads to a deceleration of the SPP wave packet, but has only 


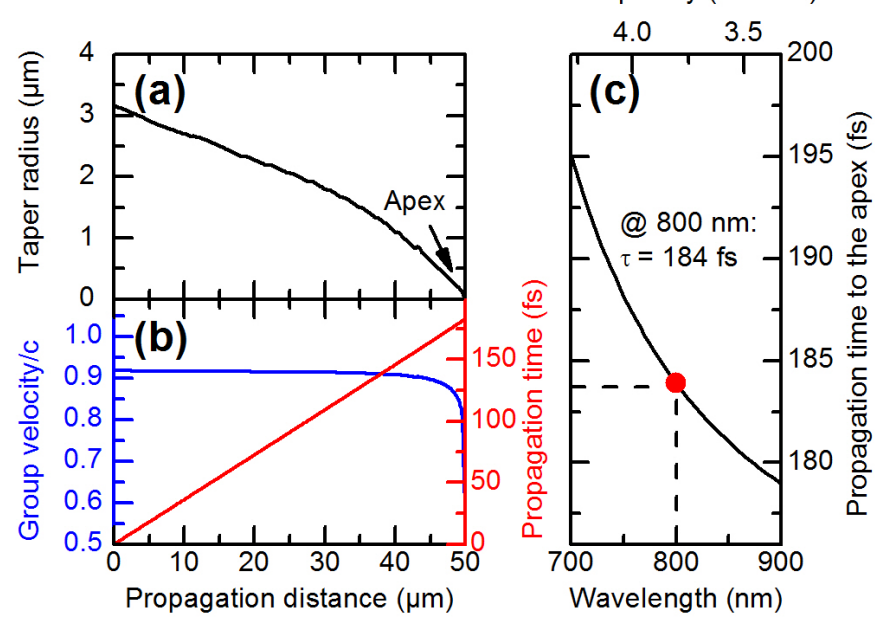

Figure 7.3: Simulation results: (a) Taper radius as a function of the propagation distance starting from the grating coupler (propagation distance $0 \mu \mathrm{m})$ and ending at the apex $(50 \mu \mathrm{m})$. The values are extracted from the SEM image shown in Fig. 7.1. (b) Plasmon velocity (blue, left scale) and propagation time (red, right scale) as a function of propagation distance (incident/SPP wavelength: $800 \mathrm{~nm} / 780 \mathrm{~nm}$ ). (c) Propagation time from the grating to the apex for different excitation wavelengths.

a marginal effect on the integrated group delay time. The simulated total propagation time from the grating to the tip apex amounts to 184 fs [Figs. 7.3(b) and 7.3(c)], which is in good agreement with our experimental results. The small difference most likely stems from deviations in the effective NIR beam path length not accounted for by the electrooptic sampling measurement, caused by, e.g., a minor relative tilt between the tip surface and the ZnTe crystal. The angular precision of the tip alignment was approximately $2^{\circ}$ corresponding to an error of 6 fs.

Figure 7.3(c) plots the dispersion of the plasmon group delay time, showing a decrease with incident wavelength that corresponds to a group delay dispersion of $24 \mathrm{fs}^{2}$ (chirp of $-0.0705 \mathrm{fs} / \mathrm{nm}$ ) at $800 \mathrm{~nm}$ wavelength. In our experiment, the spectral density of the excitation pulse has a full-width at half-maximum (FWHM) of $26 \mathrm{~nm}$. For a bandwidthlimited pulse, the increase of the pulse duration due to the group delay dispersion would be less than 1 fs and is thus negligible.

In addition to the plasmon propagation, the streaking spectrograms give information on the influence of the plasmonic excitation on the electron distribution in the gold apex of the tip. The flat horizontal feature at higher energies and negative delays in Figs. 7.2(a) and 7.2(b) arises from THz-induced hot-electron tunneling [17]. The decay of this tunneling signal for negative delays signifies the evolution of the hot electron temperature in the apex region. The almost identical tunneling signal for both excitation conditions implies very similar electron dynamics for both types of excitation.

In conclusion, we employed $\mathrm{THz}$ streaking to measure the group delay time of SPPs upon nanofocusing. Generally, the experimental approach yields access to the relaxation of hot carrier distributions, SPP propagation times and dispersion, and the duration of photoelectron pulses, key features in the application of plasmonic nanotapers for time-resolved electron imaging. In the future, THz streaking can be used to study spatiotemporal field evolutions and carrier dynamics also in other structures, such as double-ended optical antennae or plasmonic nanoarrays, mapping SPP propagation with nanometer spatial and femtosecond temporal resolution.

We gratefully acknowledge funding by the Deutsche Forschungsgemeinschaft (DFG) 
(SFB-1073, Project C4) and by the European Research Council (ERC-StG. "ULEED", Project ID: 639119). We thank Karin Ahlborn for help in tip fabrication. 


\section{Chapter 8}

\section{Discussion}

In the preceding Chapters, $\mathrm{THz}$ streaking is introduced as a versatile means for the spatiotemporal study of optically-induced near-fields and for the control of femtosecond electron pulses. In combination with a static bias, the $\mathrm{THz}$ near-field allows for the energetic reshaping of electron energy spectra and a manipulation of the electron pulse in phase space. $\mathrm{THz}$ streaking facilitates the analysis of plasmon dynamics and of plasmon-induced heating of the tip apex.

In this Chapter, the main results of this thesis are briefly summarized, and an outlook to future research topics related to $\mathrm{THz}$ streaking on nanostructures is given. Ideas for the application of $\mathrm{THz}$ near-field streaking in electron microscopy, prospects for future experiments and additional simulations are discussed.

\subsection{Summary: Aspects of THz streaking at metal nanotapers}

The two publications reprinted in the Chapters 6 and 7 represent core results of this thesis, focusing on different aspects of $\mathrm{THz}$ streaking at metal nanotapers.

Chapter 6 presents a detailed analysis of a series of spectrograms recorded at different bias voltages, complemented by numerical simulations of the spectrograms, the electron trajectories in the near-field, and the temporal evolution of the phase space density distribution.

A static voltage applied to the tip acts as an independent control handle of the electron dynamics in the $\mathrm{THz}$ near-field. The negative bias allows for electron emission also at positive $\mathrm{THz}$ field strengths and electron energies below the bias energy.

The effect of the external bias on the streaking spectrograms observed in the experiments is reproduced by simulations, which are determined by three input parameters: the surface field strength of the $\mathrm{THz}$ field and the static field, and a joint field decay length, assuming a dipolar field decay for both components. From the simulations, the characteristic parameter values of the $\mathrm{THz}$ near-field and the static field are extracted. 

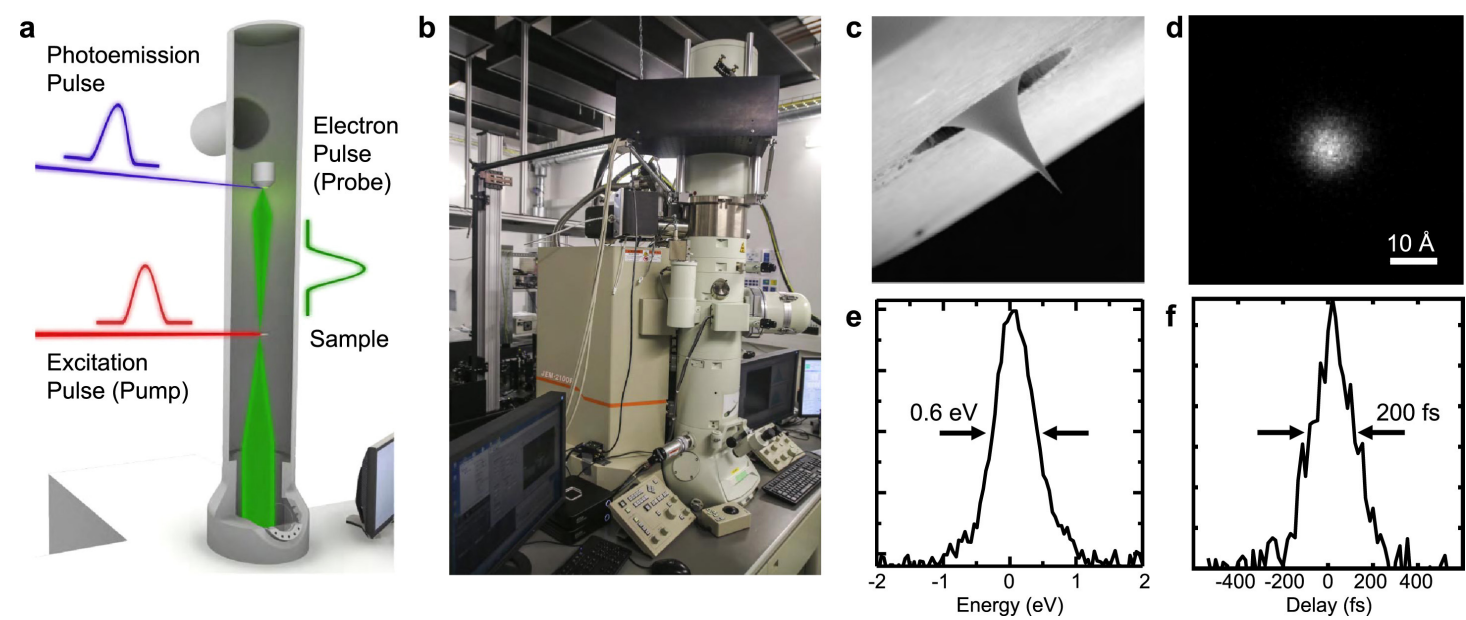

Figure 8.1: Experimental setup and beam characteristics of the Göttingen UTEM. (a) Sketch of the UTEM beam path. (b) For the implementation of time-resolved electron microscopy, a column of a JEOL JEM-2100F in combination with a Schottky emitter (c) is used. (d) The focused electron beam has a FWHM of $89 \mathrm{pm}$. (e) Energy width and (f) duration of the electron beam after the acceleration by a voltage of $200 \mathrm{kV}$ (Figure taken from Ref. [84], (C)Elsevier).

In specific emission phases, the simulated electron trajectories exhibit a caustic transfer function from the initial electron energy directly after the emission to the final energy of the electrons after leaving the near-field. For these emission phases, the temporal evolution of the phase space density distribution is evaluated, revealing a varying phase space volume of the electron pulse enabled by the spatiotemporal inhomogeneity of the THz near-field.

Within the course of the work, an analytic model is introduced to illustrate the effect of the bias voltage on the electron trajectories.

These findings build a foundation to optimize electron pulses for future applications by manipulating the electron trajectories and by reshaping the phase space density distribution.

The second study, presented in Chap. 7, employs THz streaking on plasmonic nanotapers to measure the plasmon propagation time from a grating coupler to the tip apex. The experimentally determined propagation time is in agreement with results from numerical computations. These simulations are based on a model for adiabatic nanofocusing that describes the plasmon velocity as a function of the tip diameter.

$\mathrm{THz}$ streaking at plasmonic nanotapers can measure the plasmon-induced temperature increase at the tip apex (cf. Appendix B, Sec. B.4.2) and the energy dispersion of the plasmon. Also in this experiment, the $\mathrm{THz}$ field can be exploited for the control of electron pulses on the nanoscale.

Further topics of streaking on plasmonic nanotapers are discussed in Sec. 8.3.3. 


\subsection{Application of $\mathrm{THz}$ streaking in time-resolved electron mi- croscopy}

In time-resolved electron microscopy, electron pulses are usually manipulated by bunching or deflection of the electron beam. Bunching of the electron beam shapes the energy spectrum of the electron pulse by a time-dependent electric field directed in parallel to the electron trajectories. A deflection of the electron beam by an electric field pointing in an orthogonal direction to the electron beam path induces a spatial energy chirp that allows for the selection of electrons with a specific energy by an aperture. The phase space volume of the pulse can be reduced at the cost of electron losses, caused by apertures blocking the unwanted parts of the beam (see, e.g., Ref. [169]). With these tools at hand, pulse durations in the sub-picosecond range could be reached already in 1977 by T. Hosokawa, H. Fujioka and K. Ura [170].

In Chapter. 6, the application of $\mathrm{THz}$ streaking for the control of electron pulses in time-resolved electron microscopy and diffraction experiments was suggested. In this section, the possibilities to implement $\mathrm{THz}$ streaking for the reshaping of electron pulses are discussed exemplary for an ultrafast transmission electron microscope (UTEM). The following considerations refer specifically to the UTEM setup employed in Göttingen (cf. Fig. 8.1 and Ref. [84]), but should be easily transferable to other systems.

In the Göttingen UTEM, electron pulses are emitted from a nanoscale emitter tip by femtosecond laser pulses with $400 \mathrm{~nm}$ wavelength. The electrons are accelerated by a voltage of $120-200 \mathrm{kV}$ to $60-70 \%$ of the speed of light. The electron pulses typically contain about one electron per pulse, and the pulse duration of the accumulated electron pulses is in the sub-picosecond range. So far, the shortest measured pulse duration at the sample is about $200 \mathrm{fs}$ (FWHM), which is substantially longer than the emission laser pulse duration $(\approx 50 \mathrm{fs})$. To improve the temporal resolution of the UTEM for the access to ultrafast electronic processes on the femtosecond scale, shorter pulse durations are required.

In the system in Göttingen, $\mathrm{THz}$ streaking can be applied mainly in two parts of the setup: Either directly at the tip apex by a $\mathrm{THz}$ beam co-propagating with the laser beam, similarly to the experiments demonstrated in this thesis, or the electron beam can be modified after the acceleration, e.g., by a $\mathrm{THz}$ resonator resembling the scheme presented by $C$. Kealhofer et al. in Ref. [14]. In the following, the two possibilities are compared with respect to their applicability in the setup. A general overview of the light-induced manipulation of electron pulses is given by E. Jones et al. in Ref. [171]. Besides the generation of ultrashort pulses for electron microscopy, diffraction and spectroscopy (e.g., time-resolved EELS [172]), optical control of electron pulses also can be used for linear accelerators (e.g., $[121,173,174])$. 
(a)

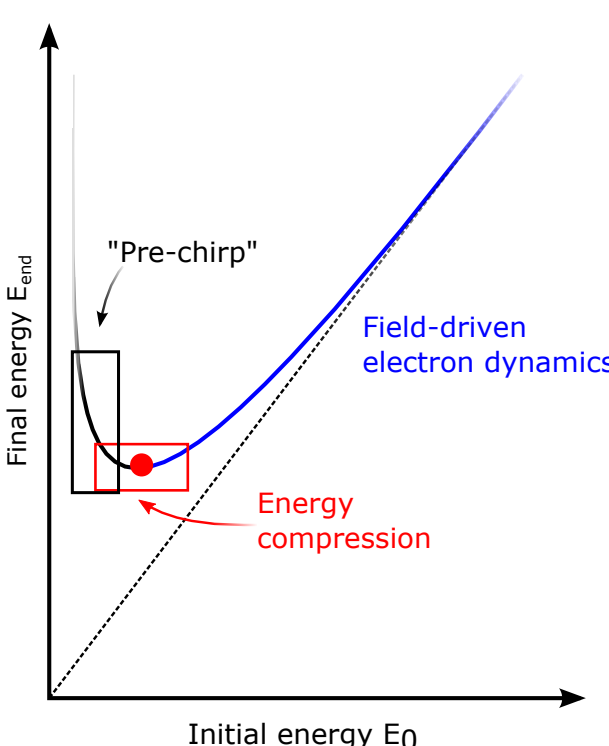

(b) At the emission time After the near-field At the sample
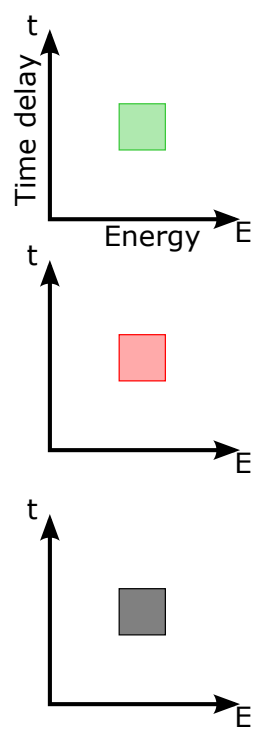
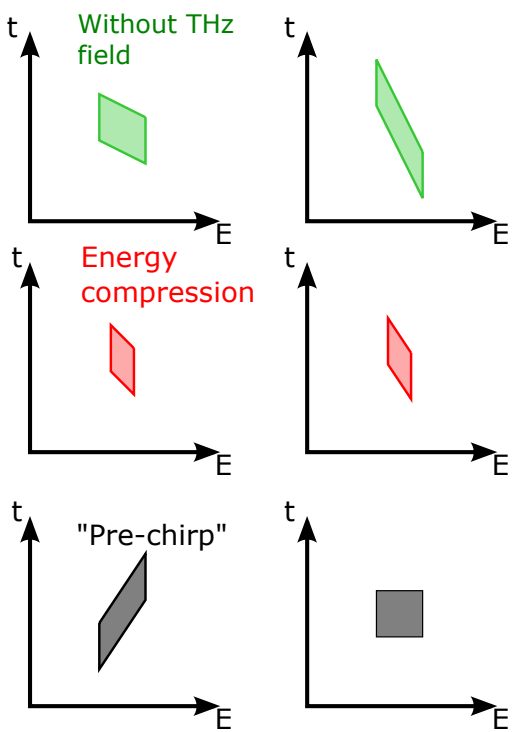

Figure 8.2: Exemplary options of electron pulse compression directly at the emitter. (a) Sketch of a caustic energy transfer function from initial energies directly after photoemission to the energy after acceleration in the $\mathrm{THz}$ near-field and the field of the static bias. (b) Energy dispersion of the electron pulse upon propagation using a square-shaped initial energy distribution as an example. Green: Without THz pulse. Red: A compression of the energy distribution in the $\mathrm{THz}$ near-field suppresses the dispersive stretching of the pulse. Black: A "Pre-chirp" induced by the THz transient compensates for the dispersion.

\subsubsection{THz streaking at the emitter}

The reshaping of the electron pulses directly at the emitter is a straight-forward application of $\mathrm{THz}$ streaking at metal nanotips. In particular, the results of our study of the electron trajectories and the phase space density distribution presented in Chap. 6 can be harnessed for the compression of the energy spectra and the minimization of the phase space volume to reduce the electron pulse duration.

The emitter tip employed in the TEM has a larger radius of curvature (about $130 \mathrm{~nm}$ ) than the tapers used in the present streaking experiments. Thus, a lower field enhancement and more pronounced caustic trajectories are expected, facilitating access to the phase space volume of the electron pulse.

The simplest approach is to employ streaking in a $\mathrm{THz}$ phase that causes caustic trajectories and use only electrons with energies close to the caustic (cf. Fig. 8.2(a), red box). Due to the spectral confinement, the dispersive stretching of the electron pulse during the propagation is reduced (cf. Fig. 8.2(b), red).

Besides a compression of the energy spectra, an energetic "pre-chirp" of the electron pulses also reduces the pulse duration at the sample, i.e., the electrons with a higher kinetic energy leave the $\mathrm{THz}$ near-field after the lower energetic electrons in such a way that the electron pulses are temporally confined to the minimum in the sample plane (cf. Fig. 8.2(b), black). Such electron trajectories are found for initial energies smaller than the initial energy of the caustic (cf. Fig. 8.2(a), black box). This approach resembles 
the compression scheme in optical resonator cavities as discussed below.

In ultrafast electron microscopy, a compression of the phase space density distribution is always advantageous, since it suppresses the dispersive energy spread, and the pulse duration is reduced below the original pulse duration. However, in conservative fields, the phase space volume of the electron pulse is preserved for a constant number of electrons (Liouville theorem, Ref. [175]). Thus, a significant reduction of the phase space volume always corresponds to large electron losses. In contrast, $\mathrm{THz}$ near-field streaking of low energy electrons allows for a control of the phase space volume while preserving the number of electrons (cf. Chap. 6, Fig. 6.9), which is enabled by a non-conservative acceleration of the electron pulses in the spatiotemporally varying near-field.

The phase space volume of the electron pulse is ultimately limited by the Heisenberg uncertainty relation and depends on the number of electrons per pulse as discussed, e.g., by J. Portman et al. in Ref. [176] or by A.H. Zewail and J.M Thomas in Ref. [169].

\subsection{2 $\mathrm{THz}$ streaking after the acceleration unit}

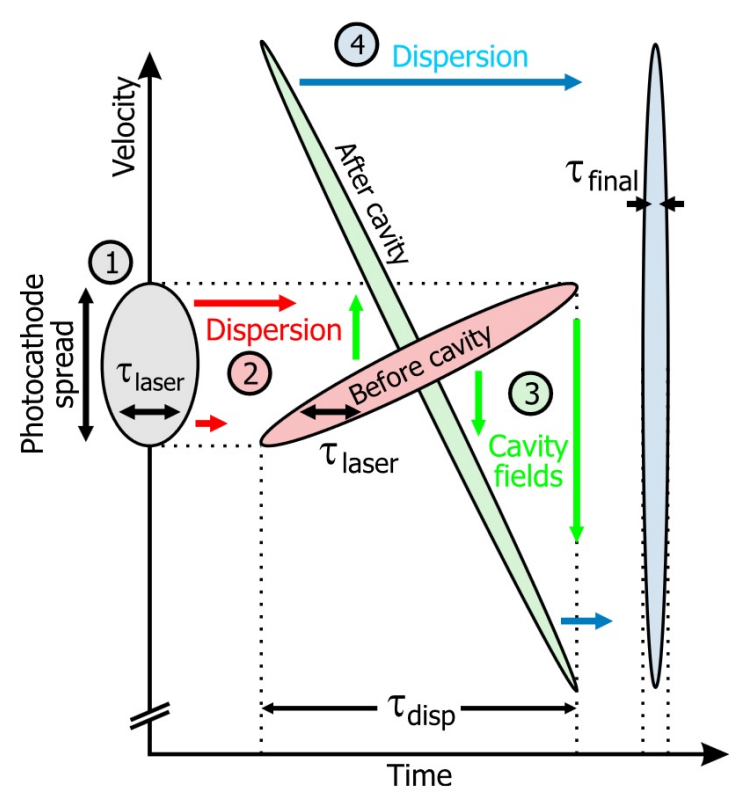

Figure 8.3: Phase space density evolution for the temporal compression of an electron pulse in an optical resonator (Figure taken from Ref. [13]).
To compress electron pulses below the original pulse duration, temporarily varying electric fields are required [13]. Electron pulses with high kinetic energies $E$ up to several $\mathrm{MeV}$ can be temporally compressed by optical resonators using radio wave, micro wave or $\mathrm{THz}$ frequencies (see, e.g., Refs. [13, 101, 177]). In these resonator cavities, an energetic chirp $\Delta E$ is imprinted onto the electron pulse that compensates for the dispersive energy spread at the sample. The temporal compression of the electron pulse comes at the cost of an energy spread. The phase space volume of the electron pulse is approximately conserved while passing through the cavity (cf. Fig. 8.3) because the energy change in the cavity is small compared to the electron velocity, i.e., $\Delta E / E$ is small and the time in the cavity field is nearly the same for all electrons in the pulse.

After the acceleration of the electrons to an energy of 120 - $200 \mathrm{keV}$ in the Göttingen UTEM, the electron pulses could be temporally compressed by a $\mathrm{THz}$ resonator following a scheme introduced by C. Kealhofer et al. (cf. Fig. 8.4). This method is based on a near-field induced at an aperture that acts as a resonator. When the electrons pass 


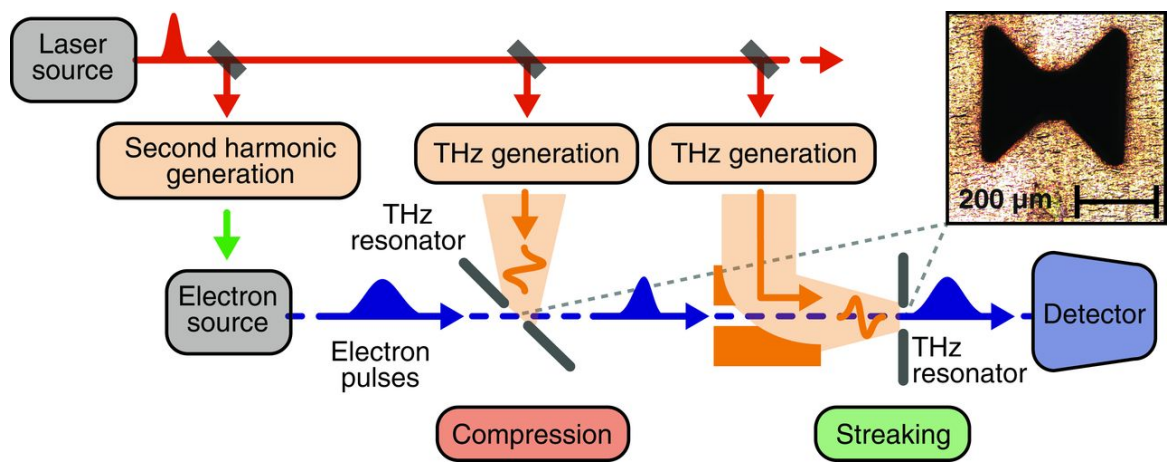

Figure 8.4: Sketch of the experimental setup implemented by Kealhofer et al. (Reprinted with permission from AAAS). From [14]. Reprinted with permission from AAAS.).

through the aperture, they are accelerated by the near-field, and their individual energy gain depends on the instant in which they enter the field. This time-dependent energy gain compensates for the energy dispersion upon propagation.

To access the phase space volume of the electron pulses, the pulse duration (or the spatial extent of the pulse) has to change during the propagation in the cavity. For a compression in phase space, the pulse duration has to decrease significantly in the microwave cavity, which could be enabled by a large temporal gradient of the field. These conditions can be reached in principle by increasing either the $\mathrm{THz}$ field strength or the resonator frequency has to be changed. For relativistic electrons, this project would be rather challenging since the conditions had to be changed quite significantly to access the phase space. It has to be considered that the complete electron pulse has to pass the aperture during the linear part of the sinusoidal field gradient, which sets an upper limit to the frequency.

An advantage of modifying the electrons after the acceleration stage is a suppression of the Coulomb effect that plays only a minor role on the short time scales of the temporal focusing of the electron pulse at the sample. In contrast, the reshaping of electron pulses directly at the emitter facilitates a compression of the phase space volume, but the quality of the electron pulses might be affected upon propagation by Coulomb repulsion in the case one uses more than one electron per pulse.

\subsection{Experimental aspects}

In this section, a number of additional experiments are proposed that complement and continue the work presented in this thesis. Ideas for experimental studies discussed in the following are

- the effect of the streaking frequency on the compression of electron pulses, 


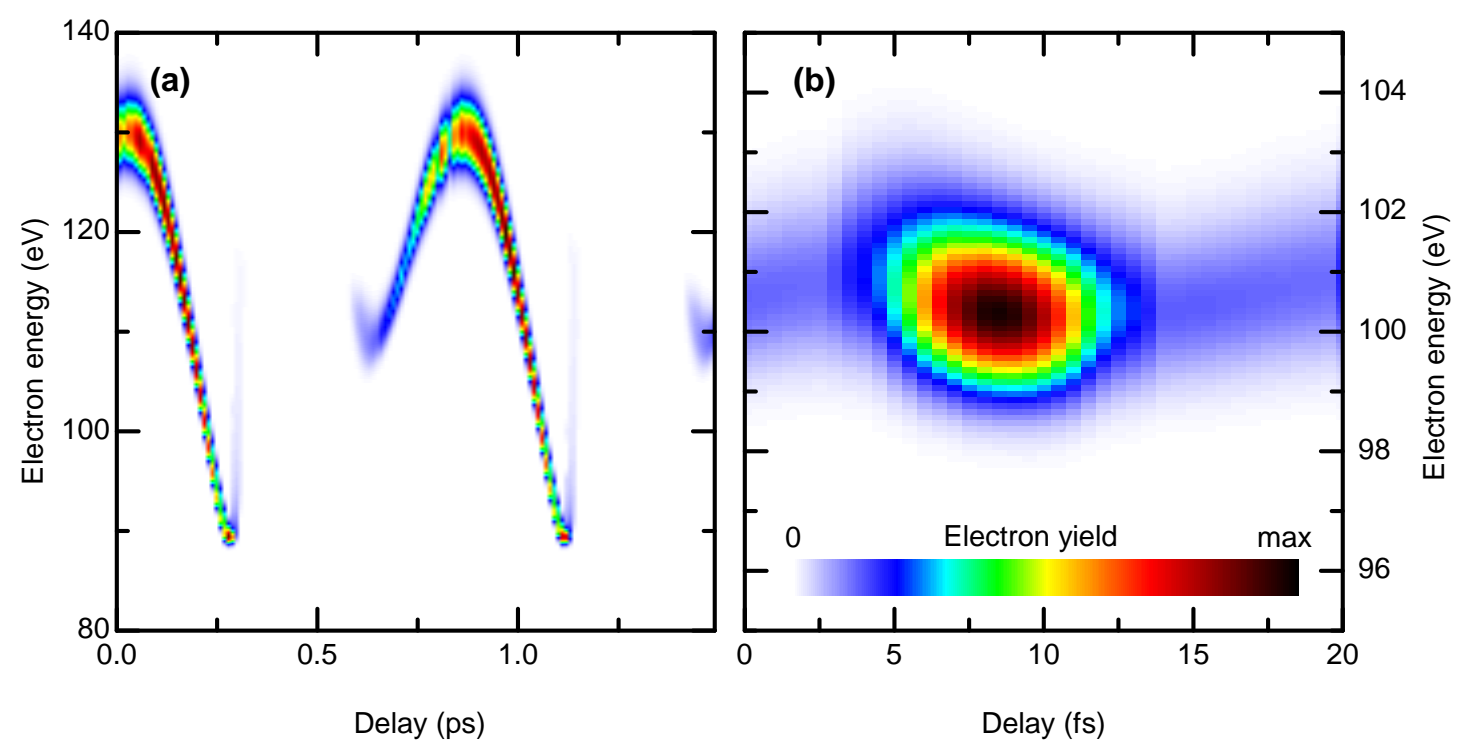

Figure 8.5: Simulation of streaking spectrograms at - $100 \mathrm{~V}$ bias voltage for $1.2 \mathrm{THz}(\mathrm{a})$ and $60 \mathrm{THz}(\mathrm{b})$ for the near-field parameter values used in Chap. 6. The energy resolution of the TOF is taken into account assuming a drift voltage of $-70 \mathrm{~V}$. Please note the different scales on the axes in (a) and (b).

- the relaxation of the NIR-induced hot electron distribution for a large variety of experimental conditions

- additional aspects of $\mathrm{THz}$ streaking on plasmonic nanotapers,

- the systematic analysis of the antenna response of the nanotip.

Besides the pure knowledge gain, the findings of these studies will help to optimize near-field driven electron pulse control.

\subsubsection{Changing the streaking frequency}

In our streaking experiments, the electron pulses are emitted by femtosecond NIR pulses and accelerated by $\mathrm{THz}$ transients. The combination of the frequencies, in this case in the NIR and the $\mathrm{THz}$ frequency range, determines the shape of the resulting spectrograms. For example, streaking measurements employing attosecond pulses in the XUV range for the emission process and NIR pulses to influence the electron trajectories, exhibit completely different electron dynamics [127, 166]. Changing the frequency can improve the capability of the streaking pulse to reshape the electron energy spectra.

For the application in time-resolved electron-probe techniques, the energy compression of electron pulses is desired to avoid a dispersive stretching of the pulses. The optimum compression is reached for a caustic transfer function from the initial electron energy to the final electron energy, i.e., different initial energies are mapped onto a common 
final energy (cf. Chap. 6). Such an energy transfer function is reached if the electrons with the initially lowest kinetic energy gain more energy in the THz near-field than the originally faster electrons, e.g., at both sides of the photocurrent suppressed interval in the spectrograms presented in Chap. 6. In THz streaking, caustic trajectories appear more pronounced for lower field strengths and longer field decay lengths.

Longer wavelengths facilitate field-driven dynamics and suppress a reshaping of the electron energy. Shorter wavelengths, e.g., in the mid-infrared range, enhance propagation effects that lead to caustic behavior because the temporal gradient of the electric field at the metal surface is usually much larger than in the $\mathrm{THz}$ range (also depending on the local intensity). In this section, the possibility of near-field streaking in the mid-IR spectral range is discussed.

For the experimental implementation, phase-stable IR pulses are required. These can be generated using a dual optical parametric amplifier (OPA), e.g., the "TOPAS twins" from LIGHT CONVERSION, which holds two separately controllable beam paths with wavelengths in the range of $1160 \mu \mathrm{m}$ to $2600 \mu \mathrm{m}$. The two beam paths both use the same white light source. Hence, the phases of the output beams are locked to each other, which allows for the generation of phase-stable difference frequency pulses, e.g., in GaSe. A detection of the carrier-envelope phase would be possible, e.g. by employing a scheme developed by C. Manzoni et al. in Ref. [178].

For mid-IR radiation, the duration of an optical cycle is in the range of $10 \mathrm{fs}(3 \mu \mathrm{m})$ to $166 \mathrm{fs}(50 \mu \mathrm{m})$. In streaking experiments, the pulse duration of the emission pulse should be short in comparison to the period of the streaking pulse. Thus, the duration of the NIR pulses (50 fs) used in the THz streaking experiments is too long for streaking in the mid-IR range and would have to be temporally compressed, e.g., by using a quartz crystal for self-phase modulation and a chirped mirror for dispersion control [179].

The experimental outcome of near-field streaking in the mid-IR range can be estimated by simulations. Figure 8.5 (a) depicts a streaking simulation for the $\mathrm{THz}$ frequency used in the experiments $(1.2 \mathrm{THz})$ with the parameter values optimized for the spectrograms in Chap. 6. In Fig. 8.5 (b), the same values are used in combination with a streaking frequency of $60 \mathrm{THz}$ corresponding to a wavelength of $5 \mu \mathrm{m}$. The two spectrograms look entirely different: The mid-IR spectrogram exhibits a less pronounced energy modulation and a strong spectral reshaping including an energy compression of the electron pulses, partially concealed by the energy resolution of the TOF spectrometer, which is included in the simulations. Note that the simulations in Fig. 8.5 (b) do not account for photoemission by the mid-IR beam.

Employing the same parameter values for the surface field strength and the spatial field decay as in the $\mathrm{THz}$ range shows the influence of the wavelength on the electron dynamics. The values in the real experiment are expected to differ from the $\mathrm{THz}$ to the mid-IR range, and the differences can be studied experimentally, providing information also about the nanotip antenna response for a broad range of frequencies.

For the application of electron pulse control via optical streaking, the local intensity 
should be estimated to select the optimal streaking frequency, considering also the expected electron velocity. Mid-IR frequencies could be an interesting option under experimental conditions similar to those found in the THz streaking experiments.

\subsubsection{THz tunneling at high intensities}

So far, we investigated $\mathrm{THz}$ tunneling for a relatively small range of NIR and $\mathrm{THz}$ intensities. Commonalities among our measured spectrograms are:

- The tunneling electrons stem from energy levels close to the Fermi-energy, indicated by the energy gap between the maximum of the streaking trace and the maximum energy of the tunneling electrons that corresponds to the work function as discussed in Ref. [17].

- The NIR intensity is far below the destruction limit to prevent a degradation of the tip that causes typically a change of the photocurrent and of the streaking trace.

These measurements can be reproduced by simulations employing the two-temperature model. The resulting temperatures are usually in the range of $1000-2000 \mathrm{~K}$. In one of our previous studies [17], we investigated the tunneling contribution in a series of spectrograms recorded at different $\mathrm{THz}$ field strengths in a moderate range and observed an enhancement of the THz-induced photocurrent upon increasing the field strength.

For both an increase of the NIR and the $\mathrm{THz}$ field strength, more tunneling electrons are emitted, and the measurements could be affected by space charge effects. If both intensities are moderately enhanced, it can be expected that the overall tunneling current and the decay time increase. In our simulations, a high electron temperature caused by intense NIR pulses leads to a tunneling emission from higher energies in the metal (see Appendix B, Sec. B.4.3), if the $\mathrm{THz}$ field strength is not increased simultaneously. In case the electrons are tunneling from energies closer to the vacuum level, the image charge effect starts to play a role for the tunneling probability and the electron trajectory. For high electron temperatures and $\mathrm{THz}$ field strengths, the photocurrent decay can be expected to deviate from the simplified two-temperature model in our simulations, especially close to the damage threshold of the tip. For example, the spatial distribution of the electron temperature, neglected in our simulations, might influence the temporal decay of the tunneling current.

The most interesting region to study in the spectrograms is directly after the NIR excitation at the maximum of the electron temperature, where the energy maximum of the streaking trace is superposed with the tunneling current. Here, also combined processes, e.g., the THz-induced tunneling of an electron that absorbed a NIR photon, could be observed. If the temporal resolution of the spectrograms, governed by the duration of the NIR pulses, is increased, the measurements could reveal time-resolved information about the non-thermal NIR-excitation of the electron system. 
A study of the tunneling current under these extreme conditions can contribute to a characterization of the heat transport in nanotapers.

For example, if we employ a high THz field strength without additional NIR excitation on a sharp nanotip, in some cases we observe an extremely high photocurrent a few seconds after opening the beam. Here, it can be assumed that the $\mathrm{THz}$ pulses transfer energy to the tip in an cumulative process.

\subsubsection{Prospects of $\mathrm{THz}$ streaking on plasmonic nanotapers}

In Chapter 7, THz streaking at plasmonic nanotapers was demonstrated and applied to measure the propagation time of the plasmons along the tip shaft. Besides the plasmon propagation velocity, THz streaking on plasmonic nanotapers reveals additional information about the processes during the plasmon propagation. In Chap. 7, a measurement of the electron temperature at the apex and the duration of the emitted electron pulse was suggested. The relaxation of the NIR-induced hot-carrier distribution is simulated in Appendix B, Sec. B.4. In Chapter 7, the duration of the electron pulse appears equal for direct apex excitation and plasmon-induced electron emission. To resolve plasmon-induced changes of the electron pulse duration, a decrease of the excitation pulse duration or an increase of the propagation path length in comparison to the experiments in Chap. 7 are required.

The investigation of the plasmon propagation for different apex radii and tip opening angles complementing previous studies on plasmon propagation $[60,61,157]$ would be a natural sequel to the study in Chap. 7. In this Section, two further project ideas will be introduced, namely the excitation of plasmonic nanotapers by multiple frequencies and the measurement of plasmon-induced changes of the tip shape.

\section{Plasmon excitation for two different frequencies}

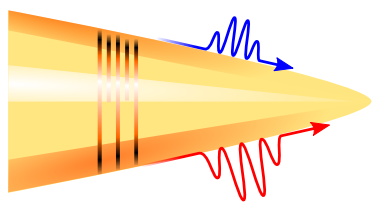

Figure 8.6: Sketch of plasmon excitation at a metal nanotip using two different excitation frequencies

Plasmonic nanofocusing allows for an isolated nanometric electron or light source on a nanotip apex applicable, e.g., in scanning probe-techniques to avoid far-field background radiation. In our experiments, the $\mathrm{THz}$ near-field was generated by focusing the $\mathrm{THz}$ beam directly onto the tip apex. For some experimental applications, the macroscopic $\mathrm{THz}$ focus surrounding the apex might be unfavorable.

For future experiments, a plasmonic in-coupling also of the long wavelength streaking pulse could circumvent this issue (see Fig. 8.6). For the experimental implementation, the focus diameter of the $\mathrm{THz}$ pulse in the range of several hundreds of micrometers has to be considered. Hence, the excitation of a THz mode (Sommerfeld-Zenneck wave, cf. Fig. 4.2 in Chap. 4 and, e.g., Ref. [180]) has to take place in a sufficient distance from the tip apex. The shape of the 
resulting streaking trace shows the long-wavelength plasmon arriving at the tip apex in a phase-resolved manner.

Employing two plasmon pulses of more similar wavelength with variable delay enables cross-correlation measurements that could reveal the temporal structure of the plasmon pulses as demonstrated in Ref. [148] and create complex electron trajectories.

\section{Measuring changes of the propagation distance}

According to experimental observations of B. Schröder (work within SFB-1073, Project C4, see also Ref. [181]), who employs plasmonic nanotapers in scanning tunneling microscopy, a nanotip expands due to plasmonic excitation by several hundreds of nanometer, depending on the NIR intensity at the grating. This effect was also described, e.g., by Grafström et al. [182] and Boneberg et al. [183]. $100 \mathrm{~nm}$ correspond to about $0.3 \mathrm{fs}$ for propagation with the speed of light, so the expected effect is at the resolution limit of our setup. It can be assumed that the effect is mainly due to an expansion of the apex region, where the adiabatic approximation does not apply. In this region, the propagation velocity of the plasmon is reduced far below the speed of light and thus, small changes in the propagation distance appear more pronounced. Therefore, changes of the delay shift between apex and grating excitation cannot be translated directly to a specific propagation velocity and distance.

Possible NIR-induced changes of the delay shift in the spectrograms could be related to an electron temperature. So far, the spatial distribution of the electron temperature is neglected in the simulations. A spatial expansion of the nanotip arises from local changes of the lattice constant and the phonon temperature. From previous studies [17], it is known that the cooling of a hot carrier distribution in a nanotip is delayed in comparison to planar surfaces, presumably due to the limited possibilities of energy spread in a confined structure. THz streaking at plasmonic nanotapers could offer an opportunity to gain more information about the spatial energy distribution in a nanotip.

The increase of the tip length as a function of the energy deposition by the incident NIR pulse could be investigated for different tip shapes in B. Schröder's experiment and compared to the results obtained by $\mathrm{THz}$ streaking.

\subsubsection{The antenna response as a function of tip radius and opening angle}

In our experiments, the main optical cycle of the $\mathrm{THz}$ transient is usually in good agreement with the electro-optic sampling measurements. After the main optical cycle, oscillations often occur in the streaking spectrograms that appear less pronounced or different in the electro-optic sampling measurements. These oscillations are not reproducible by simulations taking the electro-optic sampling measurements as an incident field. Deviations between the local incident field and the electro-optic sampling trace 
might stem from the spatial averaging over the NIR focus or the spectral limitations of measurements in ZnTe and it can be expected that antenna effects will play an additional role $[24,25]$.

The approach in Refs. [24, 25] to describe the antenna response as RLC circuit offers a practical model to characterize the response of individual nanotips. However, it does not allow for predictions of the near-field from the shape and the material of the tip to the near-field.

In our experimental setup, the antenna response of metal nanotips can be easily investigated employing a streaking spectrogram at a high bias voltage as a measure for the near-field. Using a set of tips with different opening angles and apex curvature for streaking spectrograms recorded exactly at the same position defined by the NIR spot position allows separating effects arising from the tip shape from electro-optic sampling measurement artefacts.

Variations of the tip material, e.g., using semiconductor materials or metallic alloys could lead to near-field responses, deviating from those already observed. A conical nanotaper in the $\mathrm{THz}$ focus can be considered as a semi-infinite antenna rod. Interrupting the long, conducting wire a few micrometers behind the apex might change the antenna response significantly. Changes in the conductivity affecting the antenna response of the tip can also originate from grain boundaries close to the apex region of the tip. In this context, the impact of the annealing process could be systematically analyzed in our experiment. Using streaking spectrograms recorded at different NIR spot positions, effects arising from a spatial averaging in the electro-optic sampling measurements can be distinguished from antenna effects. From test measurements used to optimize the NIR spot position, we know that the shape of the streaking trace is highly sensitive to the tip position within the $\mathrm{THz}$ focus.

\subsection{Future simulations}

In the following, two ideas on how to extend the presented simulations are discussed: an iterative algorithm that automatically determines the local $\mathrm{THz}$ field without any additional input from electro-optic sampling measurements or the streaking spectrogram itself and the inclusion of the NIR influence on the electron trajectories. Both suggestions are computationally relatively expensive and should be only applied if the present state of the simulations does not lead to satisfying results.

Further projects could be the evaluation of the electron pulses in energy and time in analogy to the phase space density. The view of the electron pulse in energy and time diagrams could be used to prepare for the experimental implementation of $\mathrm{THz}$-driven pulse control in a UTEM. 


\subsubsection{Iterative extraction of the $\mathrm{THz}$ near-field transient}

Up to now, the local $\mathrm{THz}$ transient is emulated in the simulations by a superposition of the electro-optic sampling measurements and a field-driven streaking trace, if available. The $\mathrm{THz}$ field at the position of the tip might deviate from the electro-optic sampling trace that shows the spatially averaged streaking field strength within the NIR focus. Also, the antenna response could affect the spectrum of the $\mathrm{THz}$ near-field. Thus, a field-driven streaking trace is always preferable in comparison to electro-optic sampling measurements. However, usually, the streaking trace does not cover the $\mathrm{THz}$ field in all phases and the electron kinetic energy does not exactly follow the electric field in phases of lower field strength.

Optimal simulations would use the $\mathrm{THz}$ near-field transient to compute the electron trajectories. The streaking spectrograms are unambiguously related to the optical driving field taking into account the tunneling contribution. Thus, in principle, it is not required to assume a specific incident $\mathrm{THz}$ transient to simulate the streaking spectrograms and the simulation can be used to determine the temporal evolution of the THz field. An algorithm to extract the $\mathrm{THz}$ near-field from the simulations has to compare the computations in each step with the measurement results and optimize the agreement by a variation of the near-field parameters.

\subsubsection{Influence of the $800 \mathrm{~nm}$ pulse on the electron trajectories}

For short $\mathrm{THz}$ field decay lengths and high NIR intensities, ponderomotive oscillations driven by the NIR field will presumably influence the streaking spectrograms. In principle, these oscillations can be included in the simulations. However, considering the NIR influence on the electron trajectories will significantly increase the computation time of the simulations. The NIR pulses are not phase-stable, so the trajectories have to be computed for every delay for a sufficient number of NIR carrier-envelope phases, which will multiply the computing time required for one spectrogram by the number of NIR phases. Depending on the carrier-envelope phase, the NIR pulse increases or decreases the propagation time of the electron in the near-field. Hence, a broadening of the energy spectra can be expected.

In case of a significant influence of the NIR field on the electron trajectories, the simulations might be used to estimate the NIR field strengths.

\subsection{Conclusions}

In this thesis about $\mathrm{THz}$ streaking at metal nanotips, the complex photoelectron trajectories and the temporal evolution of the electron pulse in phase space were investigated 
using a static bias as an additional control handle for the electron dynamics. THz streaking was applied to plasmonic nanotapers for a measurement of the plasmon propagation velocity. In this Section, a number of additional topics in the context of $\mathrm{THz}$ streaking were discussed, illustrating the complexity and versatility of $\mathrm{THz}$ streaking. 


\section{Appendix A}

\section{Abbreviations and symbols}

\section{Abbreviations}

$\begin{array}{ll}\text { AC } & \text { Alternating current } \\ \mathrm{BBO} & \text { Barium borate } \\ \mathrm{CEP} & \text { Carier-envelope phase } \\ \text { EELS } & \text { Electron energy loss spectroscopy } \\ \mathrm{EOS} & \text { Electro-optic sampling } \\ \mathrm{eV} & \text { Electron volt } \\ \mathrm{FWHM} & \text { Full width at half maximum } \\ \mathrm{fs} & \text { Femtosecond } \\ \mathrm{GaP} & \text { Gallium phosphide } \\ \mathrm{GaSe} & \text { Gallium selenide } \\ \mathrm{GHz} & \text { Gigahertz } \\ \mathrm{kHz} & \text { Kilohertz } \\ \mathrm{kV} & \text { Kilovolt } \\ \mathrm{MCP} & \text { Micro-channel plate } \\ \mathrm{mm} & \text { Millimeter } \\ \mu \mathrm{m} & \text { Micrometer } \\ \mathrm{ND} & \text { Neutral density } \\ \mathrm{NIR} & \text { Near-infrared } \\ \mathrm{nm} & \text { Nanometer } \\ \mathrm{ns} & \text { Nanosecond } \\ \mathrm{PHz} & \text { Petahertz } \\ \mathrm{SPP} & \text { Surface plasmon polariton } \\ \mathrm{THz} & \text { Terahertz } \\ \mathrm{TOF} & \text { Time-of-flight spectrometer } \\ \mathrm{W} & \text { Watt } \\ \mathrm{ZnTe} & \text { Zink telluride } \\ & \end{array}$




\section{Symbols}

\begin{tabular}{|c|c|}
\hline$A$ & Vector potential \\
\hline$A_{0}$ & Richardson coefficient \\
\hline$a$ & Grating period \\
\hline$a_{\max }$ & Maximum amplitude \\
\hline$a_{n}$ & Scaling factor of the photon order \\
\hline$C$ & Capacity \\
\hline$C_{e}$ & Electron heat capacity \\
\hline$C_{l}$ & Lattice heat capacity \\
\hline$c_{0}$ & Vacuum speed of light \\
\hline$\vec{D}$ & Displacement \\
\hline$d$ & Field decay length \\
\hline$d_{z}$ & Tip sample distance \\
\hline$E$ & Energy \\
\hline$E_{0}$ & Initial energy of the electrons \\
\hline$E_{\text {end }}$ & Final energy of the electrons \\
\hline$E_{F}$ & Fermi energy \\
\hline$E_{\max }$ & Maximum energy in the streaking trace \\
\hline$E_{\min }$ & Minimum energy in the streaking trace \\
\hline$E_{\text {on }}$ & Onset energy in the streaking trace \\
\hline$E_{\text {pot }}$ & Potential energy \\
\hline$e$ & Elementary charge \\
\hline$G$ & Electron phonon coupling factor \\
\hline$g$ & Grating constant \\
\hline$F$ & Electric field \\
\hline$F_{\text {stat }}^{0}$ & Static surface electric field \\
\hline$F_{\text {stat }}^{1}$ & Homogeneous component of the static electric field \\
\hline$F_{T H z}^{0}$ & $\mathrm{THz}$ surface electric field \\
\hline$I$ & Intensity \\
\hline$J$ & Photocurrent density \\
\hline$K_{e}$ & Electron thermal conductivity \\
\hline$K_{l}$ & Lattice thermal conductivity \\
\hline$\vec{k}$ & Wave vector \\
\hline$k_{b}$ & Boltzmann constant \\
\hline$k_{\text {cal }}$ & Calibration constant \\
\hline$k_{f}$ & Field factor \\
\hline$L$ & Inductance \\
\hline$l$ & Crystal thickness \\
\hline$l_{f}$ & Half-decay length of the field \\
\hline$l_{q}$ & Quiver amplitude \\
\hline
\end{tabular}




\begin{tabular}{|c|c|}
\hline$m$ & Diffraction order \\
\hline$m_{e}$ & Electron mass \\
\hline$n_{p}$ & Plasmon refractive index \\
\hline$n$ & Natural number \\
\hline$n_{1}, n_{2}$ & Material constant for metal \\
\hline$n_{e}$ & Number of electrons per unit volume \\
\hline$n_{g}$ & Group refractive index \\
\hline$P$ & Power \\
\hline$\vec{P}$ & Polarization \\
\hline$P_{\text {diss }}$ & Dissipated power \\
\hline$P_{\text {inc }}$ & Incident power \\
\hline$p$ & Momentum \\
\hline$S$ & Source term in the two-temperature model \\
\hline$s(t)$ & Trajectory \\
\hline$s_{\text {prop }}$ & Propagation length \\
\hline$R$ & Resistance \\
\hline$r$ & Taper radius \\
\hline$r_{0}$ & Radius of a nanostructure \\
\hline$T$ & Temperature \\
\hline$T_{e}$ & Electron temperature \\
\hline$T_{l}$ & Lattice temperature \\
\hline$t$ & Time \\
\hline$t_{0}$ & Emission time \\
\hline$U$ & Voltage \\
\hline$U_{\text {bias }}$ & Bias voltage \\
\hline$v_{g}$ & Group velocity \\
\hline$Z_{0}$ & Vacuum impedance \\
\hline$\beta$ & Plasmon wave vector \\
\hline$\varepsilon$ & Permittivity \\
\hline$\varepsilon_{0}$ & Vacuum permittivity \\
\hline$\varepsilon_{d}$ & Permittivity in dielectric \\
\hline$\varepsilon_{m}$ & Permittivity in metal \\
\hline$\varepsilon_{\infty}$ & Permittivity for infinite frequency \\
\hline$\gamma$ & Keldysh parameter \\
\hline$\gamma_{d}$ & Damping constant \\
\hline$\gamma_{E}$ & Euler Mascheroni constant \\
\hline$\delta$ & Adiabaticity constant \\
\hline$\hbar$ & Planck constant \\
\hline$\lambda$ & Wavelength \\
\hline$\lambda_{e f f}$ & Effective wavelength \\
\hline
\end{tabular}




$\begin{array}{ll}\lambda_{p} & \text { Plasma wavelength } \\ \omega & \text { Angular frequency } \\ \omega_{p} & \text { Plasma frequency } \\ \omega_{s p p} & \text { Surface plasmon frequency } \\ \Phi & \text { Work function } \\ \Phi_{e f f} & \text { Effective work function } \\ \rho & \text { Phase space density } \\ \varrho & \text { Reflectance } \\ \tau_{e} & \text { Relaxation time of the free electron gas } \\ \Theta & \text { Heaviside function } \\ r, \phi, z & \\ r, \phi, \theta & \text { Cylindrical coordinates } \\ x, y, z & \text { Spherical coodinates } \\ & \text { Cartesian space coordinates }\end{array}$




\section{Appendix B}

\section{Simulations}

Simulating streaking spectrograms allows for an in-depth analysis of the experimental results as discussed in Chap. 6. In the following, the structure of the simulations is explained, the capabilities of the numerical computations are examined, and information on additional aspects is provided, e.g., on the tunneling contribution in the spectrograms.

Both the streaking and tunneling spectra are simulated in two steps: First, the multiphoton or tunneling emission process is emulated and second, the propagation of the electrons is calculated in the $\mathrm{THz}$ near-field and the electric field of a static bias applied to the tip.

The NIR- and THz-induced photoemission processes are separately computed. If both pulses are temporally overlapping, in principle, mixed processes are possible. They are expected to occur only in a very small delay range and are neglected in the numerical model. The simulations also do not consider the Coulomb repulsion that plays a role for pulses with many electrons. In the present studies, the pulses contain a very small number of electrons, and the influence of the Coulomb effect on the propagating electrons is negligible.

The simulations describe the streaking experiment reduced to one dimension along the spatial axis that is collinear with the tip and the spectrometer axis. For most of the spectrograms, the results of the one-dimensional model are in good agreement with the measurements.

\section{B.1 Simulation of the emission process}

To describe the multiphoton emission process, we employ the Fowler-DuBridge model (cf. Sec. 2.2.3), which is based on the Sommerfeld model of a Fermi-distributed free electron gas. In this model, the density of states is independent of the electron energy. 


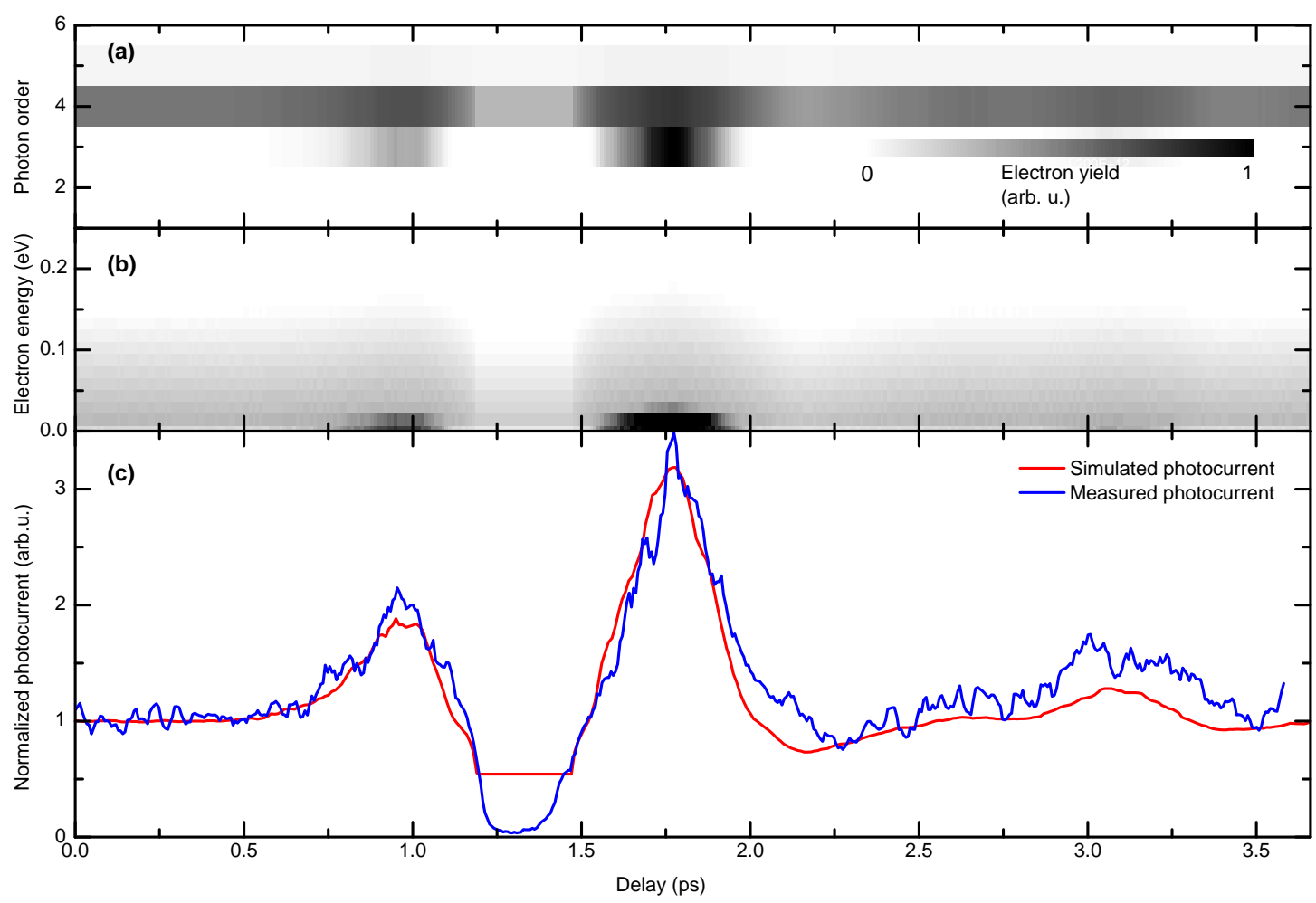

Figure B.1: Simulation of the photoemission using the Fowler-DuBridge model to reproduce the measurement presented in Chap. 6 at $U_{\text {bias }}=-100 \mathrm{~V}$. (a) Photocurrent for each photon order as a function of delay. (b) Initial kinetic energy distribution of the photoelectrons directly after the emission. (c) Simulated photoemission in comparison to the measured photocurrent. Deviations between simulation and experiment at $\approx 1.3 \mathrm{ps}$ originate from electron propagation back to the tip. This is not captured in the calculated photocurrent because the propagation is not considered, here.

The number of electrons $n_{e}$ per unit volume as a function of the velocity $\vec{v}=\left(v_{x}, v_{y}, v_{z}\right)$ is given by $[38$, p. 7$]$

$$
n_{e}\left(v_{x}, v_{y}, v_{z}\right) \mathrm{d} v_{x} \mathrm{~d} v_{y} \mathrm{~d} v_{z}=2\left(\frac{m_{e}}{h}\right)^{3} \frac{\mathrm{d} v_{x} \mathrm{~d} v_{y} \mathrm{~d} v_{z}}{e^{\left[\frac{1}{2} m_{e}\left(v_{x}^{2}+v_{y}^{2}+v_{z}^{2}\right)-E_{F}\right] / k_{b} T}+1} .
$$

Here, $m_{e}$ is the electron mass, $h$ is the Planck constant, $E_{F}$ the Fermi level, $k_{b}$ the Boltzmann constant and $T$ the temperature of the electron system. For one-dimensional emission, only the energy component in the emission direction is taken into account [38, p. 8]:

$$
n_{e}\left(v_{z}\right) \mathrm{d} v_{z}=\frac{4 \pi k_{b} T}{m_{e}}\left(\frac{m_{e}}{h}\right)^{3} \ln \left(1+e^{\left(E_{F}-m_{e} v_{z}^{2} / 2\right) / k_{b} T}\right) \mathrm{d} v_{z} .
$$

For the emission of an electron, the combined photon energy of multiple photons has to be larger than the energy difference between the electron energy and the vacuum level: 


$$
\frac{m_{e} v_{z}^{2}}{2}+n \hbar \omega \geq \Phi+E_{F}
$$

To take the Schottky effect into account, the work function $\Phi$ in Eqn. B.3 is replaced by the effective work function $\Phi_{e f f}=\Phi-\sqrt{e^{3} F_{0} / 4 \pi \varepsilon_{0}}$ (cf. Sec. 2.2.1). The electron energy component $E_{0}$ in z-direction after the emission process is given by:

$$
E_{0}=\frac{m v_{z}^{2}}{2}+n \hbar \omega-\left(\Phi_{e f f}+E_{F}\right)
$$

In the simulations, $E_{0}$ is the initial kinetic energy of the photoelectrons before propagation in the electric field surrounding the tip apex. The number of electrons with the respective energy is taken from Eqn. B.2.

This approach is implemented for different photon orders. Figure B.1(a) depicts the photocurrent for each photon order separately. As described in Sec. 2.2.3 and Eqn. 2.17, the photocurrent scales with the photon order $n$ and the factor $a_{n}$.

For the scaling factor $a_{n}$ of the photon order, no applicable values are available in literature. The scaling factor depends on the material and on a number of different physical effects not taken into account in the Fowler DuBridge model, e.g., the reflection of electrons at the surface barrier, scattering of the electrons inside the metal, etc. [38]. Therefore, the factor $a_{n}$ is obtained by a fit.

The main approximations in the simulations are the assumption of a work function for gold and the Sommerfeld model for the electron distribution.

The accurate work function of the metal, determined by the emission facet, and the distribution of the electrons $n_{e}\left(v_{z}\right)$ depends on the spatial orientation of the metal unit cell in the tip apex, which is unknown in the experiments. Hence, using an average work function of gold is an adequate first approximation. In future, the our experiment might be used for a more accurate measurement of the work function of the individual gold nanotapers. Despite its simplicity, the Sommerfeld model leads to satisfactory results reproducing the measurements. Thus, a more sophisticated approach is not required. The lowering of the work function due to the Schottky effect is determined by the surface electric field, which depends on the assumed spatial decay function of the electric field discussed in Sec. B.2. Interestingly, the experimental photocurrent cannot be reproduced employing just one photon order. The simulation of the Schottky effect indicates that for the largest negative field strength, the photon order is lowered from four to three. Including both photon orders allows for a reproduction of the experimental values as depicted in Figure B.1(c). The resulting initial energy spectra are shown in Fig. B.1(b), exhibiting higher initial energies at the maxima of the photocurrent. 


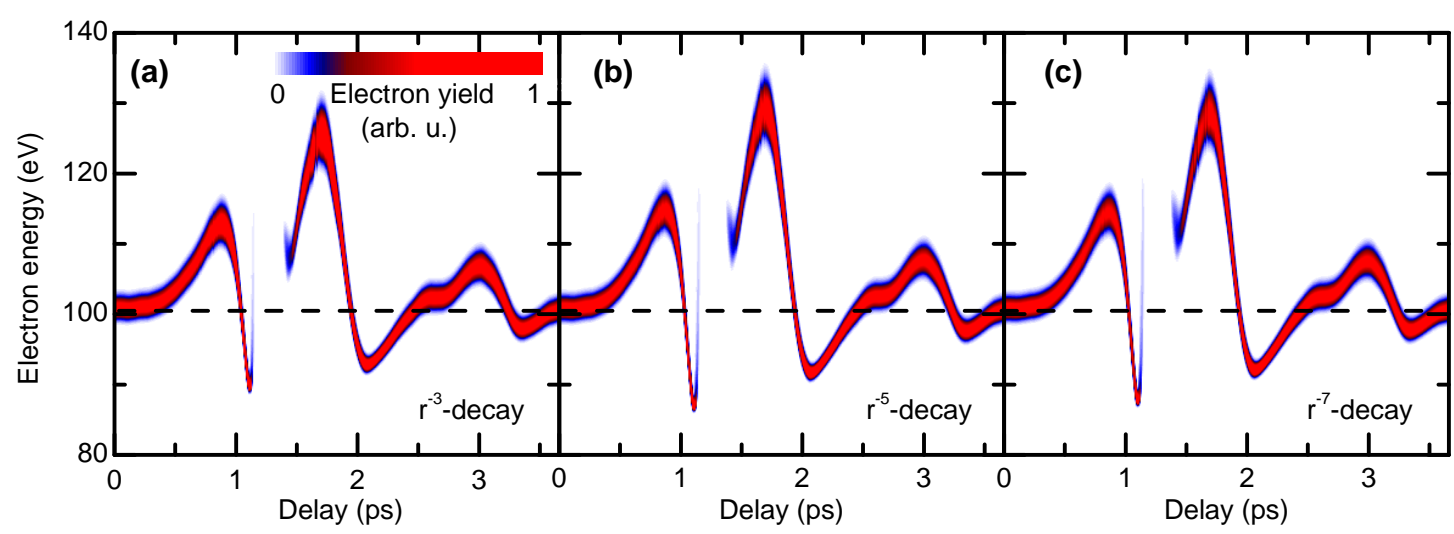

Figure B.2: Simulation of the spectrogram shown in Fig. 6.2(b) using different decay functions. The parameter values are given in Tab. B.1. (a) Dipolar decay as used in Chap. 6. (b) Quadrupolar decay. (c) Hexapolar decay.

\section{B.2 Simulation of the electron trajectories}

The electrons in the initial energy spectrum from multiphoton photoemission (cf. Sec. B.1) are propagated in the electric field of the static bias voltage. The electron motion driven by the $\mathrm{THz}$ transient and the static bias is described by the following set of equations of motion:

$$
\begin{array}{r}
\frac{d z(t)}{d t}=v_{z}(t) ; \\
\frac{d v_{z}(t)}{d t}=\frac{e}{m_{e}} F(z, t) .
\end{array}
$$

To compute the trajectories, the differential equations are solved using the Runge-Kutta algorithm provided by MATLAB.

The trajectories are computed until the electrons reach the detector in $3 \mathrm{~mm}$ distance from the tip, the propagation time exceeds a maximum value or the electrons touch the tip surface a second time after their emission (Fig. 6.4(b)), i.e., rescattering of electrons $[45,184]$ is not considered. The current simulations reproduce the measurements, and a contribution of rescattered electrons to the spectrograms is not observed.

The electric field $F(z, t)$ near the nanotip is emulated as a superposition of the $\mathrm{THz}$ electric field and the field of the static bias. As discussed in Chap. 6, the THz field is described by a purely dipolar spatial decay, and its temporal evolution is approximated by an assembly of the incident field and the kinetic energy trace for a high bias voltage (see Chap. 6, Fig. 6.8(e)). The static field employed in the simulations contains a dipolar term with a decay length equal to the $\mathrm{THz}$ field decay length and a homogeneous field component.

Analytic approximations (Sec. 2.1.1) indicate that a part of the static field decays on macroscopic length scales. Considering a plate capacitor with a nanotip attached to 


\begin{tabular}{|c|c|c|c|c|c|}
\hline$n$ & $d(\mathrm{~nm})$ & $F_{T H z}^{0}(\mathrm{MV} / \mathrm{cm})$ & $F_{\text {stat }}^{0}(\mathrm{MV} / \mathrm{cm})$ & $l_{f}(\mathrm{~nm})$ & $F_{\text {stat }}^{1}(\mathrm{~V} / \mathrm{m})$ \\
\hline \hline 3 & 212 & 3.15 & 1.45 & 55 & 282 \\
5 & 600 & 2.5 & 1.4 & 89 & 268 \\
7 & 1000 & 2.2 & 1.1 & 104 & 272 \\
\hline
\end{tabular}

Table B.1: Optimized parameter values for the simulation of streaking spectrograms using different polynomial orders.

one of the plates, the tip only influences the electric field in its close surrounding but has a negligible effect on the macroscopic homogeneous field in between the two sides of the capacitor. This assembly is roughly comparable to the situation found in the experimental setup presented in this thesis, where the electrons propagate from the tip to the grounded spectrometer entrance.

Assuming a dipolar decay for the $\mathrm{THz}$ near-field at the apex in direction of the tip axis is a reasonable approximation considering the dipolar analytic solution for spherical nanoparticles discussed in Sec. 2.1.1. The true field decay cannot be computed analytically and depends on the individual tip shape. Nevertheless, different decay functions of multipolar or exponential decay are also able to reproduce the experimental spectrograms as depicted in Fig. B.2, differing only in minor details. For reasons of physical plausibility, the dipole approximation is implemented in the simulations.

The general decay function used for the simulations in Fig. B.2 is given by:

$$
F=\left[F_{T H z}^{0}(t)+F_{\text {stat }}^{0}\right] \frac{d^{n}}{(d+x)^{n}}+F_{\text {stat }}^{1},
$$

where $n$ is the polynomial order of the decay function. The optimized parameter values employed in Fig. B.2 are given in Tab. B.1. The results show that the surface electric field decreases with increasing polynomial order.

\section{B.3 Simulation of the phase space density distribution}

In Chapter 6, a numerical analysis of the reshaping of the phase space density distribution by the spatio-temporal variations of the $\mathrm{THz}$ field was presented. In this section, these simulations are discussed in detail. Figure B.3 illustrates the steps taken in the computations.

The phase space coordinates, i.e., position and velocity of the particles, are given by the electron trajectories (cf. Fig. B.3(a)), and their time-derivative, respectively. The phase space density distribution of the whole electron pulse is calculated for a bunch of electron trajectories starting at a common emission time $t_{0}$ with a specific initial energy spectrum. The data points in phase space are weighted by the electron yield of the initial multiphoton energy spectrum (Fig. B.3(b)). A single emission time corresponds to a one-dimensional curved line (no volume!) in phase space (see Fig. B.3(d)). To compute 

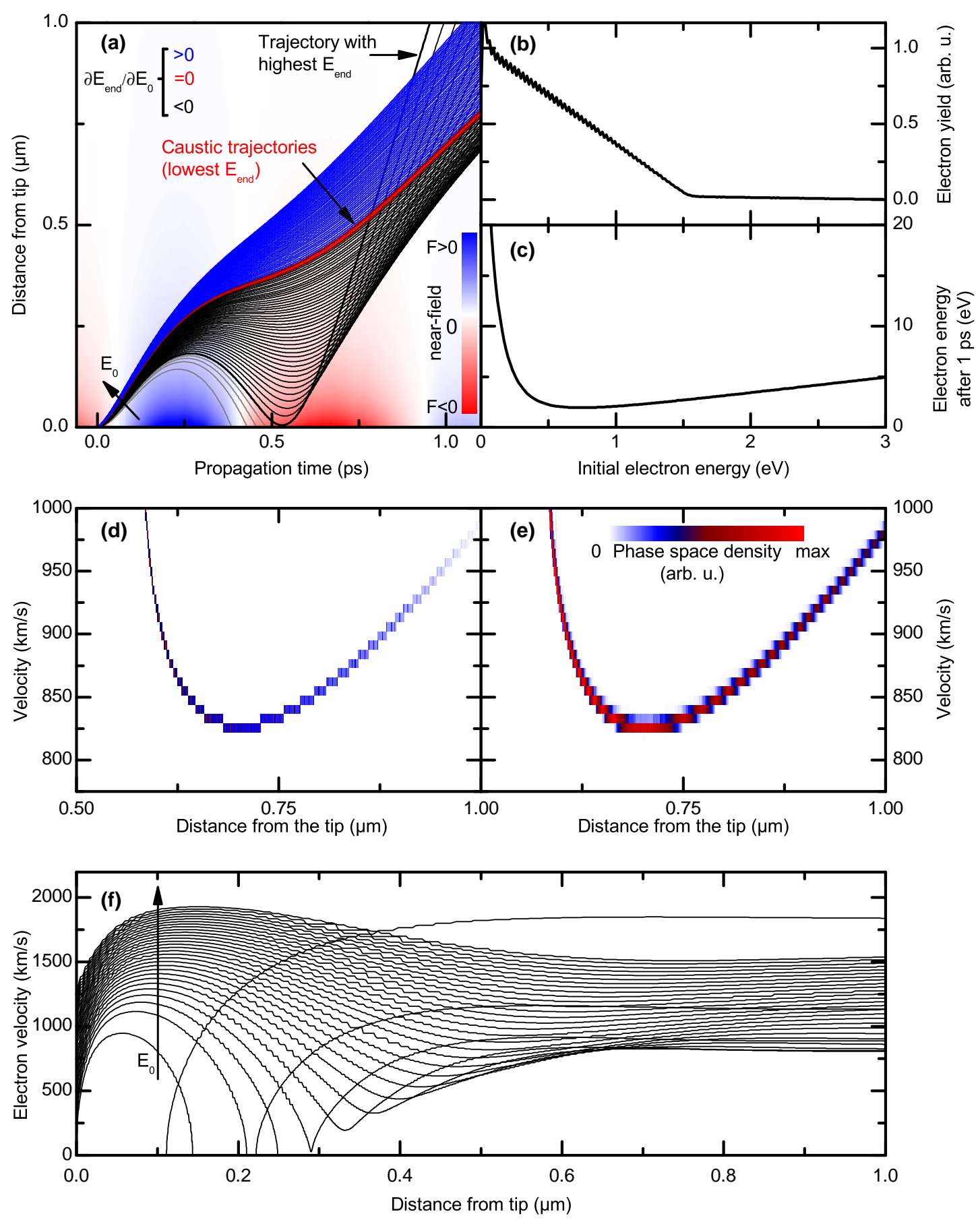

Figure B.3: Simulation of the phase space density distribution (bias: $-30 \mathrm{~V}$, emission time $t_{0}=-0.2$ ps, cf. Fig. 6.4 in Chap. 6). (a) Electron trajectories. The background color indicates the electric field (This subfigure corresponds to Fig. 6.4(b) in Chap. 6, published in Ref. [43]). (b) Initial energy spectrum simulated as described in Sec. B.1. (c) Electron energy 1 ps after the emission. (d) Phase space density distribution extracted from the trajectories at a single emission time. (e) Phase space density distribution computed from an emission interval of $30 \mathrm{fs}$. (f) Phase space trajectories. 
a physically more realistic phase space density distribution, the emitted electron bunch is emulated as a Gaussian pulse with a duration of $30 \mathrm{fs}$, and the phase space coordinates for different emission times are weighted by that Gaussian (cf. Fig. B.3(e)). In Chapter 6, Fig. 6.5, we displayed the time evolution of the phase space density distribution in a series of snapshots for propagation times $t=t_{0}+n \Delta t$. Phase space trajectories offer a complementary approach to illustrate the dynamic evolution of the electron pulse in phase space, as shown in Fig. B.3(f).

The trajectories are computed for discrete energy steps resulting in discrete data points in phase space. To emulate a smooth phase space density, the phase space is comparted in pixels and all data points in one pixel are binned together. Here, the pixel size in comparison to the phase space volume of the electron pulse is crucial for an accurate representation of the electron pulse in phase space. The number of data points in phase space is increased by interpolating trajectories for additional initial energies. The electron number in the pulse is normalized to 1.

In a conservative field, the phase space volume and the phase space density $\rho$ are constant in time for a constant particle number, which is expressed mathematically by the Liouville theorem (original publication by J. Liouville [175], helpful derivation, e.g., from H. Bradt and S. Olbert: [185]):

$$
\frac{\mathrm{d} \rho}{\mathrm{d} t}=\frac{\partial \rho}{\partial t}+\sum_{i} \frac{\partial \rho}{\partial x_{i}} \frac{\mathrm{d} x_{i}}{\mathrm{~d} t}+\sum_{i} \frac{\partial \rho}{\partial p_{i}} \frac{\mathrm{d} p_{i}}{\mathrm{~d} t}=0
$$

Here, $x_{i}$ are the spatial coordinates of the particles and $p_{i}$ are their momenta. Only if the electric field accelerating the electrons is varying in space and time (non-conservative field), the phase space volume of the electron pulse can be changed for a constant number of particles. For a compression of the electron pulse, emission times with caustic trajectories are promising candidates (see also Chap. 6, Sec. 6.10 and Fig. 6.9).

Numerical studies of the electron propagation in phase space allow for additional insights in $\mathrm{THz}$ streaking that cannot be attained experimentally and show the potential of $\mathrm{THz}$ streaking for the compression of electron pulses.

\section{B.4 Simulation of the tunneling spectra}

The THz-induced tunneling process is simulated according to the Fowler-Nordheim model (Sec. 2.2.4), employing the difference between the electron energy and the vacuum level as an effective work function for the Fermi-distributed electrons (cf. Eqn. B.2, Sec. B.1). The number of tunneling electrons depicted in Fig. B.4 is given by the product of the number of electrons with the respective effective work function and the tunneling probability described by Eqn. 2.19. 


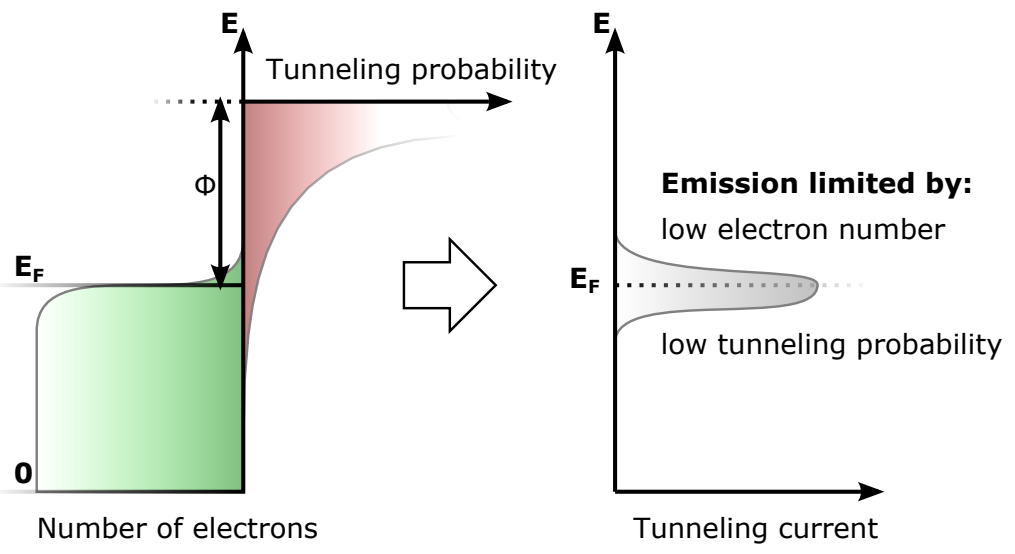

Figure B.4: Sketch of the electron distribution and the tunneling probability that determine the tunneling current.

To take into account the NIR-induced excitation of the electron distribution, the twotemperature model is used. This model was developed by S. I. Anisimov et al. (Ref. [54]) to describe the spatiotemporal evolution of the electron and the lattice temperature in a metal. The electron temperature obtained from the two-temperature model is included in the Fermi-distribution, and thus influences the tunneling current.

After the emission process, the electrons start their propagation with zero kinetic energy and at the surface of the tunneling barrier. This starting position is defined by the diameter of the tunneling barrier at the energy of the electron in the metal, i.e., no energy is transferred to the electrons during the tunneling process.

Since the tunneling electrons originate from energies close to the Fermi-energy, the image charge effect is negligible.

\section{B.4.1 Two-temperature model}

In the streaking spectrograms, the NIR pulse excites a hot electron distribution in the metal and the increased number of electrons above the Fermi-energy leads to $\mathrm{THz}$ induced tunneling already at lower THz field strengths as discussed in-depth in Sec. 3.3 and in Ref. [17]. The relaxation of the electron distribution in the spectrograms can be simulated using a two-temperature model [54], which describes the electron and the lattice temperature $T_{e}$ and $T_{l}[186]$ :

$$
\begin{array}{r}
C_{e}\left(T_{e}\right) \frac{\partial T_{e}}{\partial t}=\nabla\left[K_{e}\left(T_{e}, T_{l}\right) \nabla T_{e}\right]-G\left(T_{e}\right)\left(T_{e}-T_{l}\right)+S(\vec{r}, t), \\
C_{l} \frac{\partial T_{l}}{\partial t}=\nabla\left[K_{l}\left(T_{l}\right) \nabla T_{l}\right]+G\left(T_{e}\right)\left(T_{e}-T_{l}\right) .
\end{array}
$$

$C_{e}$ and $C_{l}$ are the electron and lattice heat capacities of the metal, $K_{e}$ and $K_{l}$ are the respective thermal conductivities, $G$ is the electron phonon coupling factor and $S$ is the source term representing the external energy supply by the laser. In our simulations, the electron temperature without additional NIR excitation is assumed to be $T_{e}=300 \mathrm{~K}$. 
The two coupled differential equations characterize the spatiotemporal evolution of the electron and the lattice temperature. The terms $\nabla\left[K_{e}\left(T_{e}, T_{l}\right) \nabla T_{e}\right]$ and $\nabla\left[K_{l}\left(T_{l}\right) \nabla T_{l}\right]$ take into account the diffusive energy transport driven by the spatial temperature gradient. In comparison to the electron heat conduction, the lattice conduction is often negligible [186]. In our experiment, the geometry of the nanotip is expected to slow down the electron diffusion [17]. Thus, we also omit the electron diffusion in our numerical simulations that now describe only the temporal evolution of the electron temperature. The term $G\left(T_{e}\right)\left(T_{e}-T_{l}\right)$ stands for the electron-phonon coupling transferring energy from the electron system to the lattice. In our simulations, we use the Sommerfeld expansion to compute the coupling factor $G=\gamma T_{e}$ with the heat capacity constant $\gamma$ [186].

The source term $S(t)$ is given by (e.g., Ref. [187]):

$$
S(t)=\sqrt{\frac{4 \ln 2}{\pi}} \frac{1-R}{\tau \delta} I_{0} \exp \left(-4 \ln 2 \frac{t^{2}}{\tau^{2}}\right) .
$$

Here, $R$ is the reflectivity of the metal, $\tau$ is the FWHM pulse duration, $\delta=1 / \alpha$ is the optical penetration depth and $\alpha$ the absorption coefficient. The material constants used in the simulation are taken from Z. Lin and V. Zhigilei (Ref. [186]) and C. Kealhofer et al. (Ref. [188]). The incident intensity is used as a free parameter that determines the electron temperature.

The two-temperature model as introduced by S. I. Anisimov et al. does not take into account ballistic transport that can be included as a small correction in the source term as described, e.g., by $S .-S$ Wellershoff (Ref. [189]), who discusses the two-temperature model in detail.

The system of differential equations Eqn. B.9 and Eqn. B.10 is solved numerically using the Euler method to obtain the electron temperature as a function of time. For each time step, the electron and the lattice temperature are computed iteratively by

$$
\begin{array}{r}
T_{e}(t+\Delta t)=T_{e}(t)+\frac{\Delta t}{C_{e}}\left[S(t)-G\left(T_{e}(t)\right)\left(T_{e}(t)-T_{l}(t)\right)\right], \text { and } \\
T_{l}(t+\Delta t)=T_{l}(t)+\frac{\Delta t G\left[T_{e}(t+\Delta t)\right]}{C_{l}}\left[T_{e}(t+\Delta t)-T_{l}(t)\right] .
\end{array}
$$

In each time step, the electron temperature is used to compute the Fermi-distribution.

\section{B.4.2 THz tunneling contribution to streaking spectrograms}

The energy spectra of the THz-emitted electrons are computed from the NIR-induced hot electron distribution to simulate the tunneling component of the spectrograms. An example for such a simulation is displayed in Fig. B.5, which is optimized to reproduce the measurements shown in Chap. 7. In the following, this example is used to explain the simulation of the tunneling contribution in the spectrograms. Figure B.6 depicts the 

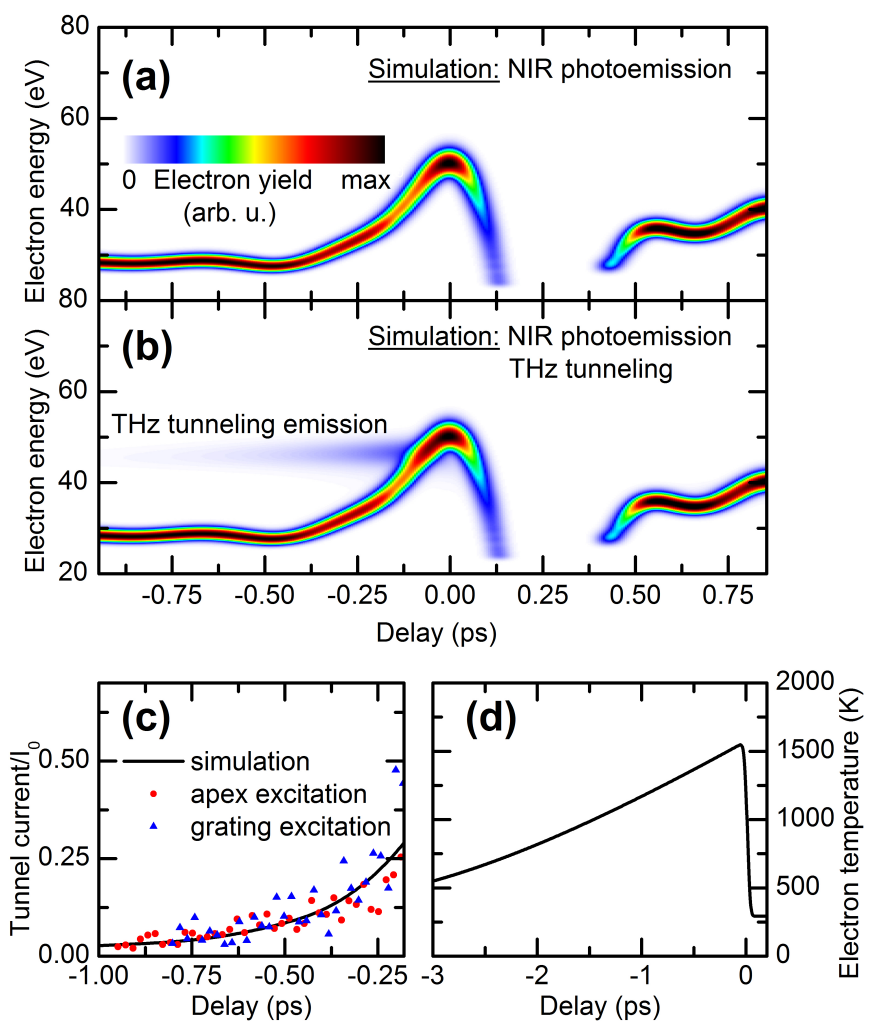

Figure B.5: Simulation of a streaking spectrogram including the tunneling contribution $\left(F_{T H z}^{0}=35 \mathrm{MV} / \mathrm{cm}, F_{\text {stat }}^{0}=15.4 \mathrm{MV} / \mathrm{cm}, l_{1 / 2}=5 \mathrm{~nm}\right)$. The parameter values are chosen to reproduce the measurements shown in Chap. 7, Fig. 7.2. (a) Simulation of the streaking spectrogram without $\mathrm{THz}$ tunneling. (b) Simulation of the spectrogram including tunneling current. (c) Tunneling current as a function of delay. Experimental and simulated data for comparison. (d) Simulation of the electron temperature relaxation.

intermediate steps and details of the simulations.

In Figure B.6(a), the electron distribution in the metal (Sommerfeld model, Fermidistribution, cf. B.1) is shown as a function of time relative to the NIR-induced excitation of the electron system, described by the source term given by Eqn. B.11 in the two-temperature model (Eqn. B.9 and Eqn B.10).

The tunneling current is computed using the Fowler-Nordheim model introduced above. In Figure B.6(b), the tunneling probability is plotted for the $\mathrm{THz}$ transient from the measurements in Chap. 7 as a function of the electron energy using the electro-optic sampling measurement from Fig. B.6(e) to describe the temporal evolution of the surface field. The small energy difference between the tunneling spectra and the energy maximum of the streaking trace in the measurements (cf. Fig. 7.2) corresponds to the work function, indicating that the tunneling electrons are emitted close to the Fermi energy. Thus, the image charge effect that only affects the potential barrier close to the vacuum level, is negligible.

To elucidate the computation of the delay-dependent tunneling current, the differences between the $\mathrm{THz}$ tunneling and the NIR-induced photocurrent are briefly reviewed in the following. Although both contributions depend on the relative pulse delay in the 


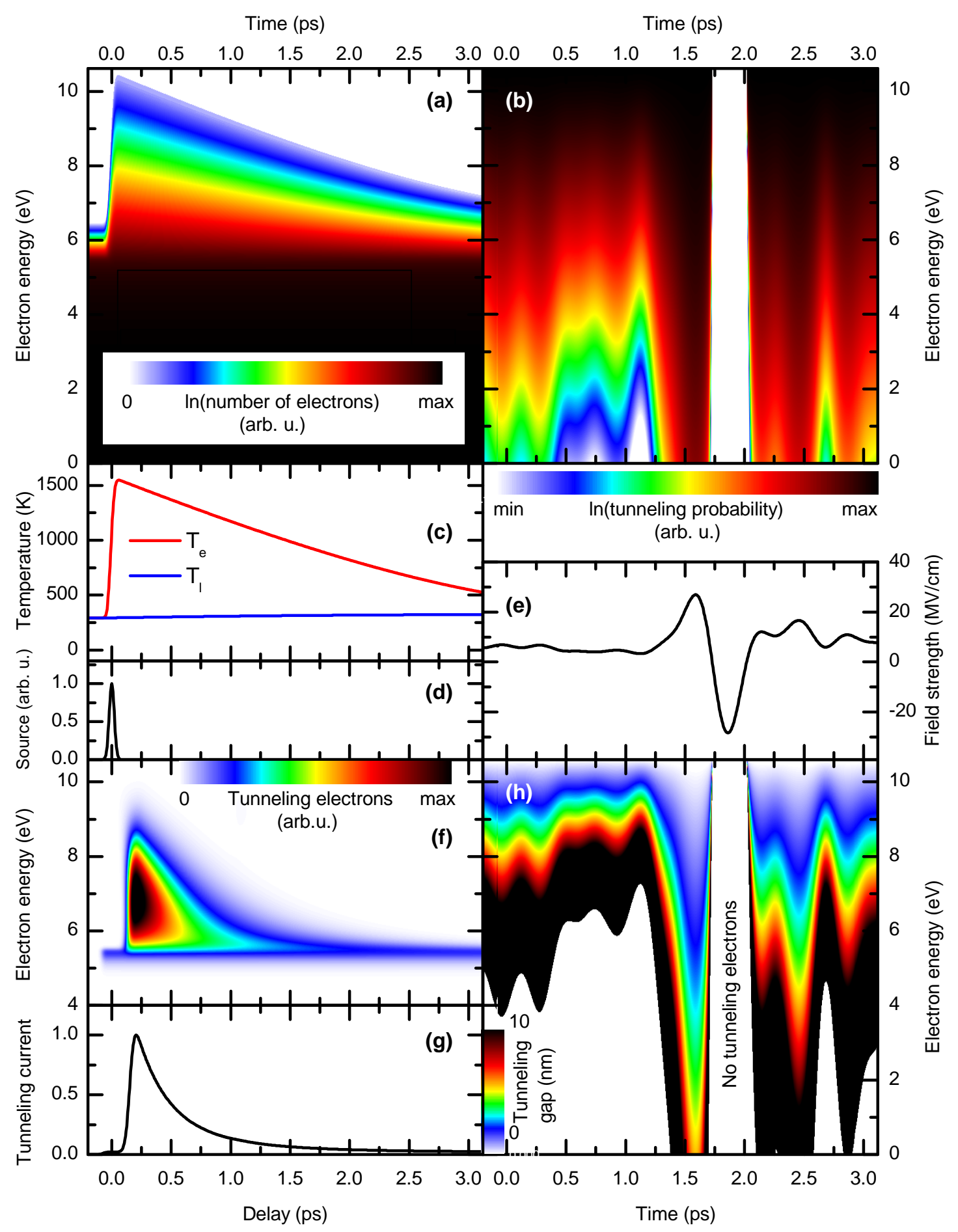

Figure B.6: Intermediate steps of the tunneling simulation. (a) Electron energy distribution in the metal. (b) Tunneling probability as a function of electron energy and emission phase in the $\mathrm{THz}$ field (e). (c) Electron and lattice temperature. (d) Energy deposition by the source term. (f) Tunneling electrons. (g) Photocurrent. (h) Tunneling barrier width. White regions indicate a gap size $>500 \mathrm{~nm}$. Gap sizes inbetween $10 \mathrm{~nm}$ and $500 \mathrm{~nm}$ are shown in black. 
streaking spectrograms, the underlying mechanisms are fundamentally different: The NIR-induced multiphoton photoemission is temporally restricted to the pulse duration of the NIR pulse and the emission phase within the $\mathrm{THz}$ transient is determined by the relative pulse delay. In contrast, the $\mathrm{THz}$ tunneling emission for a specific delay is computed as the sum over all emission phases. The THz tunneling occurs predominantly in phases of maximum negative field strength and the delay dependence of the photocurrent arises from the electron temperature in these phases.

Thus, for each electron energy, the number of tunneling electrons as a function of relative pulse delay corresponds to the convolution of the tunneling probability and the number of available electrons as depicted in Fig. B.6(f). The total THz-induced photocurrent (cf. Fig. B.6(g)), i.e. the integral of Fig. B.6(f) over all energies in the metal, is characterized by three parameters: The THz-induced photocurrent without additional NIR excitation (at positive delays), the maximum of the photocurrent and the "half-decay time".

The tunneling current amplitude in relation to the NIR-induced photocurrent is not described within our model. To include the tunneling contribution in the streaking spectra, the absolute photocurrent is adjusted to match the experimental results. This reduces the number of effectively free parameters to two.

The input parameters to compute the tunneling current are the NIR intensity that determines the strength of the source term (cf. Fig. B.6(d)), and the surface electric field, determined by the $\mathrm{THz}$ transient and the bias voltage. The parameters of the surface electric field are deduced from the simulation of the streaking spectrograms. The remaining freely adjustable parameter is the NIR intensity, which allows to extract the electron temperature from the simulations (cf. Fig. B.6(c)).

To compute the energy spectra of the tunneling electrons, the electrons are propagated in the electric field solving the same differential equations as used for multiphoton-emitted electrons for different initial conditions. The electrons start their propagation with zero initial kinetic energy at the surface of the tunneling barrier. For each emission time and electron energy, the tunneling distance is computed (cf. Fig. B.6(h)). In this way, the energy difference between the tunneling spectra and the maximum of the streaking trace is reproduced in the simulations.

The simulation of the tunneling current with the electron temperature as the only free parameter can be used as a consistency check of the streaking simulations. The simulation depicted in Fig. B.5 shows an overall good agreement with the measurement, although the incident electric field measured by electro-optic sampling deviates from the streaking trace in the second visible $\mathrm{THz}$ half-cycle. Considering the short $\mathrm{THz}$ decay length and the relatively high local NIR intensity in the tunneling simulation, including the effect of the NIR field on the electron motion could improve the simulation results (cf. Sec. 8.4). 


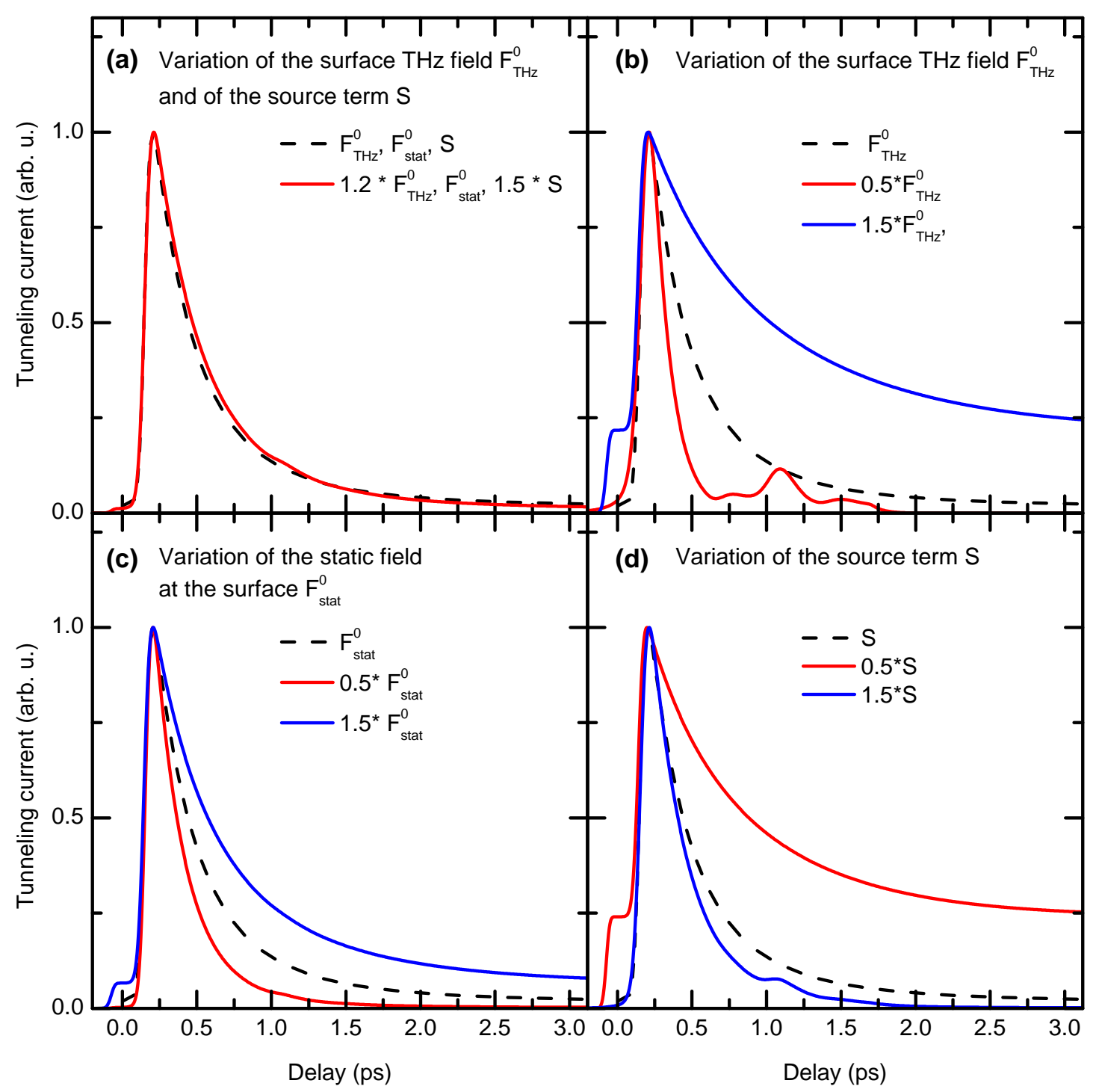

Figure B.7: Variation of the input parameter values in the tunneling simulation. (a) Optimized parameter values for the surface field strength extracted from the tunneling simulation in Fig. B.5 (black, dashed line) in comparison to an optimized tunneling current for an increased electron temperature. (b), (c), and (d) Tunneling current computed for different values of the surface $\mathrm{THz}$ field $F_{T H z}^{0}$, the static surface electric field $F_{\text {stat }}^{0}$ and the source term $S$, respectively.

\section{B.4.3 Intensity and field dependency of the $\mathrm{THz}$ tunneling simulation}

In this Section, the influence of local NIR intensity and the surface field strength of the $\mathrm{THz}$ transient and the static bias is discussed, using the tunneling current depicted in Fig. B.5(c) as a reference. Figure B.7 shows the temporal decay of the tunneling current for varying parameter values.

An increase of the NIR intensity and a decrease of the THz and static field strength have a similar effect on the tunneling simulation (cf. Fig. B.7(b) red, (c) red, and (d) blue): The photocurrent decay time decreases in comparison to the reference (black, dashed line) and tunneling does not occur without NIR excitation, e.g., at negative times or 
after the relaxation of the hot carrier distribution. The intense photocurrent peak stems from electrons tunneling directly below the vacuum level. These electrons gain the same final energy as NIR-emitted electrons at the maximum of the streaking trace, and the energy gap between streaking trace and $\mathrm{THz}$ tunneling emission vanishes. We never observed this experimentally.

If the NIR intensity declines and the surface field strengths are enhanced, the photocurrent decays more slowly in comparison to the optimized photocurrent and a large, delay-independent photocurrent contribution appears. The photocurrent is emitted from energy levels close to the Fermi level.

The decay of the tunneling current is approximately reproduced by increasing both the THz field strength and the NIR intensity (cf. Fig. B.7(a)). However, differences appear in the kinetic energy spectra of the tunneling current: A high THz surface field strength facilitates field-driven electron dynamics leading to a small spectral width of the tunneling spectra. 


\section{Bibliography}

[1] A. Einstein. Über einen die Erzeugung und Verwandlung des Lichtes betreffenden heuristischen Gesichtspunkt. Ann. Phys., 322:132-148, 1905. Cited on page 1.

[2] T. L. Cocker, D. Peller, J. Repp P. Yu, and R. Huber. Tracking the ultrafast motion of a single molecule by femtosecond orbital imaging. Nature, 539:263267, 2016. Cited on pages 1 and 44.

[3] F. Krausz and M. Ivanov. Attosecond physics. Rev. Mod. Phys., 81:163-234, 2009. Cited on pages 2, 16 and 64 .

[4] M. Hentschel, R. Kienberger, Ch. Spielmann, G. A. Reider, N. Milosevic, T. Brabec, P. Corkum, U. Heinzmann, M. Drescher, and F. Krausz. Attosecond metrology. Nature, 414:509-5013, 2001. Cited on pages 2 and 16.

[5] E. Goulielmakis, M. Uiberacker, R. Kienberger, A. Baltuska, V. Yakovlev, A. Scrinzi, Th. Westerwalbesloh, U. Kleineberg, U. Heinzmann, M. Drescher, and F. Krausz. Direct measurement of light waves. Science, 305:1267-1269, 2004. Cited on pages 2, 16, 44 and 64 .

[6] U. Frühling, M. Wieland, M. Gensch, T. Gebert, B. Schütte, M. Krikunova, R. Kalms, F. Budzyn, O. Grimm, J. Rossbach, et al. Single-shot terahertz-fielddriven X-ray streak camera. Nat. Photon., 3:523-528, 2009. Cited on pages 2, 16, 44 and 64 .

[7] B. Schütte, U. Frühling, M. Wieland, A. Azima, and M. Drescher. Electron wave packet sampling with laser-generated extreme ultraviolet and terahertz fields. Opt. Express, 19:18833-18841, 2011. Cited on pages 2, 16 and 64.

[8] S. W. Hell and J. Wichmann. Breaking the diffraction resolution limit by stimulated emission: stimulated-emission-depletion fluorescence microscopy. Opt. Lett., 19:780-782, 1994. Cited on page 2.

[9] E. Betzig, G. H. Patterson, R. Sougrat, O. W. Lindwasser, S. Olenych, J. S. Bonifacino, M. W. Davidson, J. Lippincott-Schwartz, and H. F. Hess. Imaging intracellular fluorescent proteins at nanometer resolution. Science, 313:1642-1645, 2006. Cited on page 2 .

[10] D. Courjon and C. Bainier. Near field microscopy and near field optics. Rep. Prog. Phys., 57:989, 1994. Cited on page 2.

[11] K. E. Priebe, C. Rathje, Th. Hohage S. V. Yalunin, A. Feist, S. Schäfer, and C. Ropers. Attosecond electron pulse trains and quantum state reconstruction in 
ultrafast transmission electron microscopy. Nat. Phys., 11:793-797, 2017. Cited on page 2 .

[12] Y. Morimoto and P. Baum. Diffraction and microscopy with attosecond electron pulse trains. Nat. Phys., 2017. Cited on page 2.

[13] A. Gliserin, A. Apolonski, F. Krausz, and P. Baum. Compression of single-electron pulses with a microwave cavity. New J. Phys., 14:073055, 2012. Cited on pages 2,44 and 73 .

[14] C. Kealhofer, W. Schneider, D. Ehberger, A. Ryabov, F. Krausz, and P. Baum. All-optical control and metrology of electron pulses. Science, 352:429-433, 2016. Cited on pages 2, 44, 64, 71 and 74 .

[15] L. Wimmer, G. Herink, D. R. Solli, S. V. Yalunin, K. E. Echternkamp, and C. Ropers. Terahertz control of nanotip photoemission. Nat. Phys., 10:432-436, 2014. Cited on pages 3, 13, 15, 17, 18, 19, 33, 44, 47, 51, 56, 58, 64, 66 and 117.

[16] G. Herink, D. R. Solli, M. Gulde, and C. Ropers. Field-driven photoemission from nanostructures quenches the quiver motion. Nature, 483:190-193, 2012. Cited on pages 3, 12, 44, 47, 49 and 64 .

[17] G. Herink, L. Wimmer, and C. Ropers. Field emission at terahertz frequencies: AC-tunneling and ultrafast carrier dynamic. New J. Phys., 16:123005, 2014. Cited on pages 3, 15, 20, 21, 22, 44, 47, 64, 66, 67, 77, 79, 94 and 95.

[18] L. Novotny and B. Hecht. Principles of nano-optics. Cambridge university press, 2012. Cited on pages 6 and 25 .

[19] W. Demtröder. Experimentalphysik. Springer, 2009. Cited on page 6.

[20] M. K. Miller, A. Cerezo, M. G. Hetherington, and G. C. D. W. Smith. Atom probe field microscope. Clarenton press, 1996. Cited on page 7.

[21] T. Sakurai and E. W. Müller. Field calibration using the energy distribution of field ionization. Phys. Rev. Lett., 30:532-535, 1973. Cited on page 7.

[22] T. Sakurai and E. W. Müller. Field calibration using the energy distribution of a free-space field ionization. J. Appl. Phys., 48:2618-2625, 1977. Cited on page 7.

[23] P. Bharadwaj, B. Deutsch, and L. Novotny. Optical antennas. Adv. Opt. Photon., 1:438-483, 2009. Cited on page 8.

[24] K. Wang, D. M. Mittleman, N. C. J. van der Valk, and P. C. M. Planken. Antenna effects in terahertz apertureless near-field optical microscopy. Appl. Phys. Lett., 85:2715-2717, 2004. Cited on pages 8 and 80.

[25] R. Kersting, H.-T. Chen, N. Karpowicz, and G. C. Cho. Terahertz microscopy with submicrometre resolution. Journal of Optics A: Pure and Applied Optics, 7:S184, 2005. Cited on pages 8 and 80 .

[26] H. Zhong, N. Karpowicz, and X.-C. Zhang. Terahertz emission profile from laserinduced air plasma. Appl. Phys. Lett., 88:261103, 2006. Cited on page 8.

[27] Y. S. You, T. I. Oh, and K. Y. Kim. Off-axis phase-matched terahertz emission from two-color laser-induced plasma filaments. Phys. Rev. Lett., 109:183902, 2012. Cited on page 8 . 
[28] P. Klarskov, A. C. Strikwerda, K. Iwaszczuk, and P. U. Jepsen. Experimental three-dimensional beam profiling and modeling of a terahertz beam generated from a two-color air plasma. New J. Phys., 15:075012, 2013. Cited on page 8.

[29] W. Schottky. Über kalte und warme Elektronenentladungen. Z. Phys., 14:63-106, 1923. Cited on pages 9,49 and 57 .

[30] R. Gomer. Field emission and field ionization. Harvard University press, 1961. Cited on pages 9 and 10.

[31] R. W. Boyd. Nonlinear optics. Academic Press, 2008. Cited on page 10.

[32] S. V. Keldysh. Ionization in the strong field of a strong electromagnetic wave. Sov. Phys. JETP, 20:1307-1314, 1965. Cited on page 10.

[33] R. Kopold, W. Becker, M. Kleber, and G. G. Paulus. Channel-closing effects in high-order above-threshold ionization and high-order harmonic generation. $J$. Phys. B: At., Mol. Opt. Phys., 35:217, 2002. Cited on page 10.

[34] R. H. Fowler. The analysis of photoelectric sensitivity curves for clean metals at various temperatures. Phys. Rev., 38:45-56, 1931. Cited on pages 11, 49 and 57.

[35] L. A. DuBridge. Theory of the energy distribution of photoelectrons. Phys. Rev., 43:727-741, 1933. Cited on pages 11, 49 and 57.

[36] L. A. DuBridge and R. C. Hergenrother. The effect of temperature on the energy distribution of photoelectrons. i. normal energies. Phys. Rev., 44(11):861-865, 1933. Cited on pages 11 and 57.

[37] J. H. Bechtel, W. Lee Smith, and N. Bloembergen. Two-photon photoemission from metals induced by picosecond laser pulses. Phys. Rev. B, 15:4557-4563, 10 1977. Cited on pages 11 and 57.

[38] R. B. Brogle. Studies of linear and nonlinear photoemission processes in metals and semiconductors. PhD thesis. University of California, 1996. Cited on pages $11,57,88$ and 89.

[39] R. H. Fowler and L. Nordheim. Electron emission in intense electric fields. $R$. Soc. Lond. Proc. Ser. A, 119(781):173-181, 1928. Cited on page 12.

[40] L. Wimmer, M. Sivis, G. Herink, S. V. Yalunin, K. E. Echternkamp, and C. Ropers. Ultrafast dynamics driven by intense light pulses. Ed. by M. Kitzler and S. Gräfe. Springer, 2016. Chap. Highly nonlinear and ultrafast optical phenomena in metallic nanostructures, pp. 233-258. Cited on pages 12 and 44.

[41] J. Itatani, F. Quéré, G. L. Yudin, M. Yu. Ivanov, F. Krausz, and P. B. Corkum. Attosecond streak camera. Phys. Rev. Lett., 88:173903, 2002. Cited on pages 13 and 16.

[42] F. Kelkensberg, A. Koenderink, and M. Vrakking. Attosecond streaking in a nano-plasmonic field. New J. Phys., 14:093034, 2012. Cited on pages 13, 16, 44 and 64 .

[43] L. Wimmer, O. Karnbach, G. Herink, and C. Ropers. Phase space manipulation of free-electron pulses from metal nanotips using combined terahertz near fields 
and external biasing. Phys. Rev. B, 95:165416, 2017. Cited on pages 13, 64, 66 and 92 .

[44] M. I. Stockman, M. F. Kling, U. Kleineberg, and F. Krausz. Attosecond nanoplasmonic-field microscope. Nat. Photon., 1:539-544, 2007. Cited on pages 13, 16, 44 and 64.

[45] S. V. Yalunin, G. Herink, D. R. Solli, M. Krüger, P. Hommelhoff, M. Diehn, A. Munk, and C. Ropers. Field localization and rescattering in tip-enhanced photoemission. Ann. Phys., 525:L12-L18, 2013. Cited on pages 13, 44 and 90.

[46] W. Demtröder. Laserspektroskopie. Springer Verlag Berlin Heidelberg, 2007. Cited on page 15.

[47] Alexander Horn. Ultra-fast material metrology. Viley VCH, 2009. Cited on page 15.

[48] M. Drescher, M. Hentschel, R. Kienberger, M. Uiberacker, V. Yakovlev, A. Scrinzi, Th. Westerwalbesloh, U. Kleineberg, U. Heinzmann, and F. Krausz. Time-resolved atomic inner-shell spectroscopy. Nature, 419:803-807, 2002. Cited on page 16.

[49] R. Kienberger, E. Goulielmakis, M. Uiberacker, A. Baltuska, V. Yakovlev, F. Bammer, A. Scrinzi, Th. Westerwalbesloh, U. Kleineberg, U. Heinzmann, et al. Atomic transient recorder. Nature, 427:817-821, 2004. Cited on page 16.

[50] E. Goulielmakis, V. S. Yakovlev, A. L. Cavalieri, M. Uiberacker, V. Pervak, A. Apolonski, R. Kienberger, U. Kleineberg, and F. Krausz. Attosecond control and measurement: lightwave electronics. Science, 317:769-775, 2007. Cited on page 16.

[51] E. Skopalová, D. Y. Lei, T. Witting, C. Arrell, F. Frank, S. A. Maier Y. Sonnefraud, J. W. G. Tisch, and J. P. Marangos. Numerical simulation of attosecond nanoplasmonic streaking. New J. Phys., 13:083003, 2011. Cited on pages 16 and 64.

[52] F. Süßmann and M. F. Kling. Attosecond nanoplasmonic streaking of localized fields near metal nanospheres. Phys. Rev. B, 84:121406, 2011. Cited on pages 16, 44 and 64 .

[53] A. G. Borisov, P. M. Echenique, and A. K. Kazansky. Attostreaking with metallic nano-objects. New J. Phys., 14:023036, 2012. Cited on pages 16 and 64 .

[54] S. I. Anisimov, B. Kapeliovich, and T. L. Perel'man. Electron emission from metal surfaces exposed to ultrashort laser pulses. JETP, 39:375-377, 1974. Cited on pages 23 and 94 .

[55] S. A. Maier. Plasmonics: fundamentals and applications. 1. edition, New York: Springer, 2007. Cited on pages 25, 26, 27, 28, 29 and 63.

[56] C. Noguez and C. E. Román-Velázquez. Dispersive force between dissimilar materials: Geometrical effects. Phys. Rev. B, 70:195412, 2004. Cited on page 26.

[57] M. G. Ramchandani. Energy band structure of gold. J. Phys. C: Solid State Phys., 3:S1, 1970. Cited on page 26. 
[58] M. V. Bashevoy, F. Jonsson, A. V. Krasavin, N. I. Zheludev, Y. Chen, and M. I. Stockman. Generation of traveling surface plasmon waves by free-electron impact. Nano Lett., 6:1113-1115, 2006. Cited on page 28.

[59] B. Schröder, M. Sivis, R. Bormann, S. Schäfer, and C. Ropers. An ultrafast nanotip electron gun triggered by grating-coupled surface plasmons. Appl. Phys. Lett., 107:231105, 2015. Cited on pages 28, 63, 64 and 65.

[60] B. Schröder, Th. Weber, S. V. Yalunin, Th. Kiel, C. Matyssek, M. Sivis, S. Schäfer, F. von Cube, S. Irsen, K. Busch, C. Ropers, and S. Linden. Real-space imaging of nanotip plasmons using electron energy loss spectroscopy. Phys. Rev. $B$, 92:085411, 2015. Cited on pages 28, 64 and 78 .

[61] N. Talebi, W. Sigle, R. Vogelgesang, M. Esmann, S. F. Becker, C. Lienau, and P.A. van Aken. Excitation of mesoscopic plasmonic tapers by relativistic electrons: phase matching versus eigenmode resonances. ACS Nano, 9:7641-7648, 2015. Cited on pages 28, 64 and 78 .

[62] C. Ropers, C. C. Neacsu, T. Elsaesser, M. Albrecht, M. B. Raschke, and C. Lienau. Grating-coupling of surface plasmons onto metallic tips: A nanoconfined light source. Nano Lett., 7:2784-2788, 2007. Cited on pages 29 and 63.

[63] E. Kretschmann and H. Raether. Notizen: Radiative decay of non radiative surface plasmons excited by light. Z. Naturforschung, 23:2135-2136, 1968. Cited on page 29.

[64] A. Otto. Excitation of nonradiative surface plasma waves in silver by the method of frustrated total reflection. Z. Phys. A: Hadrons nucl., 216:398-410, 1968. Cited on page 29 .

[65] R. W. Wood. XLII. On a remarkable case of uneven distribution of light in a diffraction grating spectrum. Philos. Mag., 4:396-402, 1902. Cited on page 29.

[66] E. Verhagen, L. Kuipers, and A. Polman. Enhanced nonlinear optical effects with a tapered plasmonic waveguide. Nano Lett., 7:334-337, 2007. Cited on pages 30 and 63 .

[67] A. P. Hibbins. Grating coupling of surface plasmon polaritons at visible and microwave frequencies: $\mathrm{PhD}$ thesis. University of Exeter, 1999. Cited on page 30.

[68] A. N. Sprafke. Optische Nahfeld-Wechselwirkungen von Plasmonen mit ihrer Umgebung. PhD thesis. RWTH Aachen, 2014. Cited on page 30.

[69] M. I. Stockman. Nanofocusing of optical energy in tapered plasmonic waveguides. Phys. Rev. Lett., 93:137404, 2004. Cited on pages 30, 31, 63 and 66.

[70] M. I. Stockman. Erratum: nanofocusing of optical energy in tapered plasmonic waveguides [Phys. Rev. Lett. 93, 137404 (2004)]. Phys. Rev. Lett., 106:019901, 2011. Cited on page 31.

[71] S. V. Yalunin, B. Schröder, and C. Ropers. Theory of electron energy loss near plasmonic wires, nanorods, and cones. Phys. Rev. B, 93:115408, 2016. Cited on pages 31, 63 and 66 . 
[72] B. Schröder. Lichtlokalisierung durch resonante Anregung von OberflächenPlasmonen an Metallspitzen. MA thesis. Georg-August University of Göttingen, 2013. Cited on page 33.

[73] H. G. Roskos, M. D. Thomson, M. Kreß, and T. Löffler. Broadband THz emission from gas plasmas induced by femtosecond optical pulses: From fundamentals to applications. Laser $\& 3$ Photonics Reviews, 1:349-368, 2007. Cited on page 35.

[74] D. J. Cook and R. M. Hochstrasser. Intense terahertz pulses by four-wave rectification in air. Opt. Lett., 25:1210-1212, 2000. Cited on pages 35 and 65.

[75] S. Casalbuoni, H. Schlarb, B. Schmidt, P. Schmüser, B. Steffen, and A. Winter. Numerical studies on the electro-optic detection of femtosecond electron bunches. Phys. Rev. ST Accel. Beams, 11:072802, 2008. Cited on pages 40 and 41.

[76] S. R. Tripathi, M. Aoki, T. Asahi, I. Hosako, and N. Hiromoto. Accurate optical constants of ZnTe measured by THz-TDS with their standard deviations. 35th International Conference on Infrared, Millimeter, and Terahertz Waves. 2010, pp. 1-2. Cited on page 40.

[77] O. Bostanjoglo and D. Otte. High speed transmission electron microscopy of laser quenching. Mat. Sc. Eng., 173:407-411, 1993. Cited on page 43.

[78] B. J. Siwick, J. R. Dwyer, R. E. Jordan, and R. J. D. Miller. An atomic-level view of melting using femtosecond electron diffraction. Science, 302:1382-1385, 2003. Cited on pages 43 and 44.

[79] P. Baum, D.-S. Yang, and A. H. Zewail. 4D visualization of transitional structures in phase transformations by electron diffraction. Science, 318:788-792, 2007. Cited on page 43.

[80] M. Gulde, S. Schweda, G. Storeck, M. Maiti, H. K. Yu, A. M. Wodtke, S. Schäfer, and C. Ropers. Ultrafast low-energy electron diffraction in transmission resolves polymer/graphene superstructure dynamics. Science, 345:200-204, 2014. Cited on pages 43,44 and 64 .

[81] T. LaGrange, M. R. Armstrong, K. Boyden, C. G. Brown, G. H. Campbell, J. D. Colvin, W. J. DeHope, A. M. Frank, D. J. Gibson, F. V. Hartemann, et al. Single-shot dynamic transmission electron microscopy. Appl. Phys. Lett., 89:044105, 2006. Cited on page 43.

[82] D. J. Flannigan, B. Barwick, and A. H. Zewail. Biological imaging with 4D ultrafast electron microscopy. PNAS, 107:9933-9937, 2010. Cited on page 43.

[83] L. Piazza, D. J. Masiel, T. LaGrange, B. W. Reed, B. Barwick, and F. Carbone. Design and implementation of a fs-resolved transmission electron microscope based on thermionic gun technology. Chem. Phys, 423:79-84, 2013. Cited on page 43 .

[84] A. Feist, N. Bach, N. Rubiano da Silva, T. Danz, M. Möller, K. E. Priebe, T. Domröse, J. G. Gatzmann, S. Rost, J. Schauss, S. Strauch, R. Bormann, M. Sivis, S. Schäfer, and C. Ropers. Ultrafast transmission electron microscopy using a 
laser-driven field emitter: femtosecond resolution with a high coherence electron beam. Utramicroscopy, 176:63-73, 2016. Cited on pages 43, 70 and 71.

[85] M. Eichberger, H. Schäfer, M. Krumova, M. Beyer, J. Demsar, H. Berger, G. Moriena, G. Sciaini, and R. J. D. Miller. Snapshots of cooperative atomic motions in the optical suppression of charge density waves. Nature, 468:799-802, 2010. Cited on page 44 .

[86] A. Feist, K. E. Echternkamp, J. Schauss, S. V. Yalunin, S. Schäfer, and C. Ropers. Quantum coherent optical phase modulation in an ultrafast transmission electron microscope. Nature, 521:200-203, 2015. Cited on page 44.

[87] K. E. Echternkamp, A. Feist, S. Schäfer, and C. Ropers. Ramsey-type phase control of free-electron beams. Nat. Phys., 12:1000-1004, 2016. Cited on page 44.

[88] P. Hommelhoff, C. Kealhofer, and M. A. Kasevich. Ultrafast electron pulses from a tungsten tip triggered by low-power femtosecond laser pulses. Phys. Rev. Lett., 97:247402, 2006. Cited on page 44.

[89] C. Ropers, D. R. Solli, C. P. Schulz, C. Lienau, and T. Elsaesser. Localized multiphoton emission of femtosecond electron pulses from metal nanotips. Phys. Rev. Lett., 98:043907, 2007. Cited on pages 44 and 64.

[90] B. Barwick, D. J. Flannigan, and A. H. Zewail. Photon-induced near-field electron microscopy. Nature, 462:902-906, 2009. Cited on page 44.

[91] R. Bormann, M. Gulde, A. Weismann, S. V. Yalunin, and C. Ropers. Tipenhanced strong-field photoemission. Phys. Rev. Lett., 105:147601, 2010. Cited on pages 44 and 64 .

[92] H. Yanagisawa, M. Hengsberger, D. Leuenberger, M. Klöckner, C. Hafner, Th. Greber, and J. Osterwalder. Energy distribution curves of ultrafast laser-induced field emission and their implications for electron dynamics. Phys. Rev. Lett., 107:087601, 2011. Cited on pages 44 and 64.

[93] A. Paarmann, M. Gulde, M. Müller, S. Schäfer, S. Schweda, M. Maiti, C. Xu, Th. Hohage, F. Schenk, C. Ropers, and R. Ernstorfer. Coherent femtosecond low-energy single-electron pulses for time-resolved diffraction and imaging: A numerical study. J. Appl. Phys., 112:113109, 2012. Cited on page 44.

[94] D. J. Park, B. Piglosiewicz, S. Schmidt, H. Kollmann, M. Mascheck, and C. Lienau. Strong field acceleration and steering of ultrafast electron pulses from a sharp metallic nanotip. Phys. Rev. Lett., 109:244803, 2012. Cited on pages 44 and 64 .

[95] B. Piglosiewicz, S. Schmidt, D. J. Park, J. Vogelsang, P. Groß, C. Manzoni, P. Farinello, G. Cerullo, and C. Lienau. Carrier-envelope phase effects on the strongfield photoemission of electrons from metallic nanostructures. Nat. Phot., 8:3742, 2014. Cited on page 44.

[96] M. Krüger, S. Thomas, M. Förster, and P. Hommelhoff. Self-probing of metal nanotips by rescattered electrons reveals the nano-optical near-field. J. Phys. B, 47:124022, 2014. Cited on page 44. 
[97] M. Müller, A. Paarmann, and R. Ernstorfer. Femtosecond electrons probing currents and atomic structure in nanomaterials. Nat. Comm., 5:5292, 2014. Cited on page 44 .

[98] R. Bormann, S. Strauch, S. Schäfer, and C. Ropers. An ultrafast electron microscope gun driven by two-photon photoemission from a nanotip cathode. J. Appl. Phys., 118(17):173105, 2015. Cited on page 44.

[99] D. Ehberger, J. Hammer, M. Eisele, M. Krüger, J. Noe, A. Högele, and P. Hommelhoff. Highly coherent electron beam from a laser-triggered tungsten needle tip. Phys. Rev. Lett., 114:227601, 2015. Cited on page 44.

[100] T. van Oudheusden, E. F. de Jong, S. B. van der Geer, W. E. P. M. Op 't Root, O. J. Luiten, and B. J. Siwick. Electron source concept for single-shot sub-100 fs electron diffraction in the $100 \mathrm{keV}$. J. Appl. Phys., 102:093501, 2007. Cited on page 44 .

[101] T. van Oudheusden, P. L. E. M. Pasmans, S. B. van der Geer, M. J. de Loos, M. J. van der Wiel, and O. J. Luiten. Compression of subrelativistic space-chargedominated electron bunches for single-shot femtosecond electron diffraction. Phys. Rev. Lett., 105:264801, 2010. Cited on pages 44 and 73.

[102] A. Gliserin, M. Walbran, F. Krausz, and P. Baum. Sub-phonon-period compression of electron pulses for atomic diffraction. Nat. Comm., 6:8723, 2015. Cited on page 44 .

[103] E. Curry, S. Fabbri, P. Musumeci, and A. Gover. THz-driven zero-slippage IFEL scheme for phase space manipulation. New J. Phys., 18:113045, 2016. Cited on page 44 .

[104] F. J. García de Abajo, A. Asenjo-Garcia, and M. Kociak. Multiphoton absorption and emission by interaction of swift electrons with evanescent light fields. Nano Lett., 10(10):1859-1863, 2010. Cited on page 44.

[105] S. T. Park, M. Lin, and A. H. Zewail. Photon-induced near-field electron microscopy (PINEM): theoretical and experimental. New J. Phys., 12:123028, 2010. Cited on page 44 .

[106] P. Baum and A. H. Zewail. Attosecond electron pulses for 4D diffraction and microscopy. PNAS, 104:18409-18414, 2007. Cited on page 44.

[107] T. Kampfrath, K. Tanaka, and K. A. Nelson. Resonant and nonresonant control over matter and light by intense terahertz transients. Nat. Photon., 7:680-690, 2013. Cited on page 44.

[108] A. Leitenstorfer, K. A. Nelson, K. Reimann, and K. Tanaka. Focus on nonlinear terahertz studies. New J. Phys., 16:045016, 2014. Cited on page 44.

[109] O. Schubert, M. Hohenleutner, F. Langer, B. Urbanek, C. Lange, U. Huttner, D. Golde, T. Meier, M. Kira, S. W. Koch, and R. Huber. Sub-cycle control of terahertz high-harmonic generation by dynamical Bloch oscillations. Nat. Photon., 8:119-123, 2014. Cited on page 44. 
[110] T. L. Cocker, V. Jelic, M. Gupta, S. J.Molesky, J. A. J. Burgess, G. De Los Reyes, L. V. Titova, Y. Y. Tsui, M. R. Freeman, and F. A. Hegmann. An ultrafast terahertz scanning tunnelling microscope. Nat. Photon., 7:620-625, 2013. Cited on page 44 .

[111] M. Eisele, T. L. Cocker, M. A. Huber, M. Plankl, L. Viti, D. Ercolani, L. Sorba, M. S. Vitiello, and R. Huber. Ultrafast multi-terahertz nano-spectroscopy with sub-cycle temporal resolution. Nat. Photon., 8:841-845, 2014. Cited on page 44.

[112] S. V. Yalunin, M. Gulde, and C. Ropers. Strong-field photoemission from surfaces: Theoretical approaches. Phys. Rev. B, 84:195426, 2011. Cited on page 44.

[113] M. Krüger, M. Schenk, and P. Hommelhoff. Attosecond control of electrons emitted from a nanoscale metal tip. Nature, 475:78-81, 2011. Cited on page 44.

[114] S. Zherebtsov, T. Fennel, J. Plenge, E. Antonsson, I. Znakovskaya, A. Wirth, O. Herrwerth, F. Suszmann, C. Peltz, I. Ahmad, et al. Controlled near-field enhanced electron acceleration from dielectric nanospheres with intense few-cycle laser fields. Nat. Phys., 7:656-662, 2011. Cited on page 44.

[115] M. Krüger, M. Schenk, M. Förster, and P. Hommelhoff. Attosecond physics in photoemission from a metal nanotip. J. Phys. B, 45:074006, 2012. Cited on pages 44 and 49.

[116] P. Dombi, A. Hörl, P. Rácz, I. Márton, A. Trügler, J. R. Krenn, and U. Hohenester. Ultrafast strong-field photoemission from plasmonic nanoparticles. Nano Lett., 13:674-678, 2013. Cited on page 44.

[117] F. Süßmann, L. Seiffert, S. Zherebtsov, V. Mondes, J. Stierle, M. Arbeiter, J. Plenge, P. Rupp, C. Peltz, A. Kessel, S. A. Trushin, B. Ahn, D. Kim, C. Graf, E. Rühl, M. F. Kling, and T. Fennel. Field propagation-induced directionality of carrier-envelope phase-controlled photoemission from nanospheres. Nat. Comm., 6:7944, 2015. Cited on page 44.

[118] K. Iwaszczuk, M. Zalkovskij, A. C. Strikwerda, and P. U. Jepsen. Nitrogen plasma formation through terahertz-induced ultrafast electron field emission. Optica, 2:116-123, 2015. Cited on page 44.

[119] S. Li and R. R. Jones. High-energy electron emission from metallic nano-tips driven by intense single-cycle terahertz pulses. Nat. Comm., 7:13405, 2016. Cited on page 44 .

[120] W. R.Huang, E. A. Nanni, K. Ravi, K.-H. Hong, A. Fallahi, L. J. Wong, P. D. Keathley, L. E. Zapata, and F. X. Kärtner. Toward a terahertz-driven electron gun. Sci. Rep., 5:14899, 2015. Cited on page 44.

[121] E. A. Nanni, W. R. Huang, K.-H. Hong, K. Ravi, A. Fallahi, G. Moriena, R. J. D. Miller, and F. X. Kärtner. Terahertz-driven linear electron acceleration. Nat. Comm., 6:8486, 2015. Cited on pages 44 and 71.

[122] A. Fallahi, M. Fakhari, A. Yahaghi, M. Arrieta, and F. X. Kärtner. Short electron bunch generation using single-cycle ultrafast electron guns. Phys. Rev. Accel. Beams, 19:081302, 2016. Cited on page 44. 
[123] W. R. Huang, A. Fallahi, X. Wu, H. Cankaya, A-L. Calendron, K. Ravi, D. Zhang, E. A. Nanni, K.-H. Hong, and F. X. Kärtner. Terahertz-driven, all-optical electron gun. Optica, 3:1209-1212, 2016. Cited on page 44.

[124] G. H. Kassier, N. Erasmus, K. Haupt, I. Boshoff, R. Siegmund, S. M. M. Coelho, and H. Schwoerer. Photo-triggered pulsed cavity compressor for bright electron bunches in ultrafast electron diffraction. Appl. Phys. B, 109:249-257, 2012. Cited on page 44 .

[125] J. Fabiańska, G. Kassier, and Th. Feurer. Split ring resonator based THz-driven electron streak camera featuring femtosecond resolution. Sci. Rep., 4:5645, 2014. Cited on page 44.

[126] A. Ryabov and P Baum. Electron microscopy of electromagnetic waveforms. Science, 353:374-377, 2016. Cited on page 44.

[127] B. Förg, J. Schötz, F. Süßmann, M. Förster, M. Krüger, B. Ahn, W. A. Okell, K. Wintersperger, S. Zherebtsov, A. Guggenmos, et al. Attosecond nanoscale near-field sampling. Nat. Comm., 7:11717, 2016. Cited on pages 44, 64 and 75.

[128] P. B. Corkum. Plasma perspective on strong-field multiphoton ionization. Phys. Rev. Lett., 71:1994-1997, 1993. Cited on page 49.

[129] G. G. Paulus, W. Becker, and H. Walther. Classical rescattering effects in twocolor above-threshold ionization. Phys. Rev. A, 52:4043, 1995. Cited on page 49.

[130] See Supplemental Material at http://link.aps.org/supplemental/10.1103/ PhysRevB.95.165416 for movies of the spectrograms as a function of bias and electron pulse shaping in phase space. Cited on pages 50 and 53.

[131] W. L. Barnes, A. Dereux, and T. W. Ebbesen. Surface plasmon subwavelength optics. Nature, 424:824-830, 2003. Cited on page 63.

[132] J. A. Schuller, E. S. Barnard, W. Cai, Y. C. Jun, J. S. White, and M. L. Brongersma. Plasmonics for extreme light concentration and manipulation. Nat. Mater., 9:193-204, 2010. Cited on page 63.

[133] W. Cai, W. Shin, S. Fan, and M. L. Brongersma. Elements for plasmonic nanocircuits with three-dimensional slot waveguides. Adv. Mat., 22:5120-5124, 2010. Cited on page 63.

[134] A. Kriesch, S. P. Burgos, D. Ploss, H. Pfeifer, H. A. Atwater, and U. Peschel. Functional plasmonic nanocircuits with low insertion and propagation losses. Nano Lett., 13:4539-4545, 2013. Cited on page 63.

[135] K. C. Y. Huang, M.-K. Seo, T. Sarmiento, Y. Huo, J. S. Harris, and M. L. Brongersma. Electrically driven subwavelength optical nanocircuits. Nat. Photon., 8:244-249, 2014. Cited on page 63.

[136] C. Rewitz, G. Razinskas, P. Geisler, E. Krauss, S. Goetz, M. Pawłowska, B. Hecht, and T. Brixner. Coherent control of plasmon propagation in a nanocircuit. Phys. Rev. Applied, 1:014007, 1 2014. Cited on page 63. 
[137] Y. Gorodetski, A. Niv, V. Kleiner, and E. Hasman. Observation of the spin-based plasmonic effect in nanoscale structures. Phys. Rev. Lett., 101:043903, 2008. Cited on page 63.

[138] G. Spektor, D. Kilbane, A. K. Mahro, B. Frank, S. Ristok, L. Gal, P. Kahl, D. Podbiel, S. Mathias, H. Giessen, et al. Revealing the subfemtosecond dynamics of orbital angular momentum in nanoplasmonic vortices. Science, 355:1187-1191, 2017. Cited on page 63.

[139] A. J. Babadjanyan, N. L. Margaryan, and K. V. Nerkararyan. Superfocusing of surface polaritons in the conical structure. J. Appl. Phys., 87:3785-3788, 2000. Cited on page 63.

[140] E. Verhagen, M. Spasenović, A. Polman, and L. Kuipers. Nanowire plasmon excitation by adiabatic mode transformation. Phys. Rev. Lett., 102:203904, 2009. Cited on page 63 .

[141] S. Berweger, J. M. Atkin, R. L. Olmon, and M. B. Raschke. Light on the tip of a needle: plasmonic nanofocusing for spectroscopy on the nanoscale. J. Phys. Chem. Lett., 3:945-952, 2012. Cited on page 63.

[142] M. W. Vogel and D. K. Gramotnev. Adiabatic nano-focusing of plasmons by metallic tapered rods in the presence of dissipation. Phys. Lett. A, 363:507-511, 2007. Cited on page 63.

[143] D. K. Gramotnev, M. W. Vogel, and M. I. Stockman. Optimized nonadiabatic nanofocusing of plasmons by tapered metal rods. J. Appl. Phys., 104:034311, 2008. Cited on page 63.

[144] V. Kravtsov, J. M. Atkin, and M. B. Raschke. Group delay and dispersion in adiabatic plasmonic nanofocusing. Opt. Lett., 38:1322-1324, 2013. Cited on pages 63 and 64.

[145] F. De Angelis, G. Das, P. Candeloro, M. Patrini, M. Galli, A. Bek, M. Lazzarino, I. Maksymov, C. Liberale, L. C. Andreani, et al. Nanoscale chemical mapping using three-dimensional adiabatic compression of surface plasmon polaritons. Nature Nanotechnol., 5:67-72, 2010. Cited on page 63.

[146] C. C. Neacsu, S. Berweger, R. L. Olmon, L. V. Saraf, C. Ropers, and M. B. Raschke. Near-Field Localization in Plasmonic Superfocusing: A Nanoemitter on a Tip. Nano Lett., 10:592-596, 2010. Cited on pages 63 and 64.

[147] S. Berweger, J. M. Atkin, X. G. Xu, R. L. Olmon, and M. B. Raschke. Femtosecond nanofocusing with full optical waveform control. Nano Lett., 11:4309-4313, 2011. Cited on page 63.

[148] S. Schmidt, B. Piglosiewicz, D. Sadiq, J. Shirdel, J. S. Lee, P. Vasa, N. Park, D.S. Kim, and C. Lienau. Adiabatic nanofocusing on ultrasmoothsingle-crystalline gold tapers creates a 10 -nm-sized light source with few-cycle time resolution. ACS Nano, 6:6040-6048, 2012. Cited on pages 63, 64 and 79. 
[149] V. Kravtsov, R. Ulbricht, J. M. Atkin, and M. B. Raschke. Plasmonic nanofocused four-wave mixing for femtosecond near-field imaging. Nat. Nanotechnol., 11:459464, 2016. Cited on page 63.

[150] M. Müller, V. Kravtsov, A. Paarmann, M. B. Raschke, and R. Ernstorfer. nanofocused plasmon-driven sub-10 fs electron point source. ACS Photonics, 3:611-619, 2016. Cited on pages 63 and 64.

[151] S. Berweger, J. M. Atkin, R. L. Olmon, and M. B. Raschke. Adiabatic tip-plasmon focusing for nano-raman spectroscopy. J. Phys. Chem. Lett., 1:3427-3432, 2010. Cited on page 63.

[152] D. Sadiq, J. Shirdel, J. S. Lee, E. Selishcheva, N. Park, and C. Lienau. Adiabatic nanofocusing scattering-Type optical nanoscopy of individual gold nanoparticles. Nano Lett., 11:1609-1613, 2011. Cited on page 63.

[153] A. Giugni, A. Toma B. Torre, M. Francardi, M. Malerba, A. Alabastri, R. Proietti Zaccaria, M. I. Stockman, and E. Di Fabrizio. Hot-electron nanoscopy using adiabatic compression of surface plasmons. Nat. Nano., 8:845-852, 2013. Cited on page 63 .

[154] S. Lüneburg, M. Müller, A. Paarmann, and R. Ernstorfer. Microelectrode for energy and current control of nanotip field electron emitters. Appl. Phys. Lett., 103:213506, 2013. Cited on page 64.

[155] J. Hoffrogge, J. P. Stein, M. Krüger, M. Förster, J. Hammer, D. Ehberger, P. Baum, and P. Hommelhoff. Tip-based source of femtosecond electron pulses at $30 \mathrm{keV}$. J. Appl. Phys., 115:094506, 2014. Cited on page 64.

[156] G. Storeck, S. Vogelgesang, M. Sivis, S. Schäfer, and C. Ropers. Nanotip-based photoelectron microgun for ultrafast LEED. Struct. Dyn., 4:044024, 2017. Cited on page 64 .

[157] J. Vogelsang, J. Robin, B. J. Nagy, P. Dombi, D. Rosenkranz, M. Schiek, P. Groß, and C. Lienau. Ultrafast electron emission from a sharp metal nanotaper driven by adiabatic nanofocusing of surface plasmons. Nano Lett., 15:4685-4691, 2015. Cited on pages 64 and 78 .

[158] P. Hommelhoff, Y. Sortais, A. Aghajani-Talesh, and M. A. Kasevich. Field emission tip as a nanometer source of free electron femtosecond pulses. Phys. Rev. Lett., 96:077401, 2006. Cited on page 64.

[159] B. Barwick, C. Corder, J. Strohaber, N. Chandler-Smith, C . Uiterwaal, and H. Batelaan. Laser-induced ultrafast electron emission from a field emission tip. New Journal of Physics, 9:142, 2007. Cited on page 64.

[160] M. Schenk, M. Krüger, and P. Hommelhoff. Strong-field above-threshold photoemission from sharp metal tips. Phys. Rev. Lett., 105:257601, 2010. Cited on page 64 . 
[161] S. Schmidt, P. Engelke, B. Piglosiewicz, M. Esmann, S. F. Becker, K. Yoo, N. Park, C. Lienau, and P. Groß. Wave front adaptation using a deformable mirror for adiabatic nanofocusing along an ultrasharp gold taper. Opt. Express, 21:26564-26577, 2013. Cited on page 64.

[162] M. Esmann, S. F. Becker, B. B. da Cunha, J. H. Brauer, R. Vogelgesang, P. Groß, and C. Lienau. k-space imaging of the eigenmodes of sharp gold tapers for scanning near-field optical microscopy. Beilstein J. Nanotechnol., 4:603-610, 2013. Cited on page 64.

[163] B. Wild, L. Cao, Y. Sun, B. P. Khanal, E. R. Zubarev, S. K. Gray, N. F. Scherer, and M. Pelton. Propagation lengths and group velocities of plasmons in chemically synthesized gold and silver nanowires. ACS Nano, 6:472-482, 2012. Cited on page 64 .

[164] C. Rewitz, Th. Keitzl, P. Tuchscherer, J.-S. Huang, P. Geisler, G. Razinskas, B. Hecht, and T. Brixner. Ultrafast plasmon propagation in nanowires characterized by far-field spectral interferometry. Nano Lett., 12:45-49, 2012. Cited on page 64.

[165] M. Wulf, A. de Hoogh, N. Rotenberg, and L. Kuipers. Ultrafast plasmonics on gold nanowires: confinement, dispersion, and pulse propagation. ACS Photonics, 1:1173-1180, 2014. Cited on page 64.

[166] J. Schötz, B. Förg, M. Förster, W. A. Okell, M. I. Stockman, F. Krausz, P. Hommelhoff, and M. F. Kling. Reconstruction of nanoscale near fields by attosecond streaking. IEEE Journal of Selected Topics in Quantum Electronics, 3:16516262, 2017. Cited on pages 64 and 75.

[167] M. Sandtke and L. Kuipers. Slow guided surface plasmons at telecom frequencies. Nat. Photonics, 1:573-576, 2007. Cited on page 65.

[168] P. B. Johnson and R. W. Christy. Optical constants of the noble metals. Phys. Rev. B, 6:4370-4379, 1972. Cited on page 66.

[169] A. H. Zewail and J. M. Thomas. 4D Electron microscopy: imaging in space and time. Imperial College Press, 2010. Cited on pages 71 and 73.

[170] T. Hosokawa, H. Fujioka, and K. Ura. Generation and measurement of subpicosecond electron beam pulses. Rev. Sci. Instrum., 49:624-628, 1978. Cited on page 71.

[171] E. Jones, M. Becker, J. Luiten, and H. Batelaan. Laser control of electron matter waves. Laser \& Photonics Reviews, 10:214-229, 2016. Cited on page 71.

[172] W. Verhoeven, J. F. M. van Rens, M. A. W. van Ninhuijs, W. A. F. Toonen, E. R. Kieft snd P. H. A. Mutsaers, and O. J. Luiten. Time-of-flight electron energy loss spectroscopy using TM110 deflection cavities. Struct. Dyn., 3:054303, 2016. Cited on page 71.

[173] T. Plettner, P. P. Lu, and R. L. Byer. Proposed few-optical cycle laser-driven particle accelerator structure. Phys. Rev. ST Accel. Beams, 9:111301, 2006. Cited on page 71 . 
[174] E. A Peralta, K. Soong, R. J. England, E. R. Colby, Z. Wu, B. Montazeri, C. McGuinness, J. McNeur, K. J. Leedle, D. Walz, et al. Demonstration of electron acceleration in a laser-driven dielectric microstructure. Nature, 503:91-94, 2013. Cited on page 71.

[175] J. Liouville. Note on the theory of the variation of arbitrary constants. J. Math. Pure. Appl., 3:342-349, 1838. Cited on pages 73 and 93.

[176] J. Portman, H. Zhang, Z. Tao, K. Makino, M. Berz, P. M. Duxbury, and C.Y. Ruan. Computational and experimental characterization of high-brightness beams for femtosecond electron imaging and spectroscopy. Appl. Phys. Lett., 103(25):253115, 2013. Cited on page 73.

[177] E. Curry, S. Fabbri, P. Musumeci, and A. Gover. Simulation of 3-D effects in THzbased phase space manipulation. Nucl. Instrum. Methods Phys. Res., Sect. A, 865(Supplement C). Physics and Applications of High Brightness Beams 2016:6770, 2017. Cited on page 73.

[178] C. Manzoni, M. Först, H. Ehrke, and A. Cavalleri. Single-shot detection and direct control of carrier phase drift of midinfrared pulses. Opt. Lett., 35:757-759, 2010. Cited on page 76 .

[179] M. Seidel, G. Arisholm, J. Brons, V. Pervak, and O.Pronin. All solid-state spectral broadening: an average and peak power scalable method for compression of ultrashort pulses. Opt. Express, 24:9412-9428, 2016. Cited on page 76.

[180] M. Wächter, M. Nagel, and H. Kurz. Frequency-dependent characterization of $\mathrm{THz}$ Sommerfeld wave propagation on single-wires. Opt. Express, 13:1081510822, 2005. Cited on page 78.

[181] K. Kaiser. Implementierung und Charakterisierung gepulster optischer Anregung des Spitze-Probe-Übergangs im Rastertunnelmikroskop. MA thesis. Georg-August Göttingen, 2017. Cited on page 79.

[182] S. Grafström, P. Schuller, J. Kowalski, and R. Neumann. Thermal expansion of scanning tunneling microscopy tips under laser illumination. Journal of Applied Physics, 83:3453-3460, 1998. Cited on page 79.

[183] J. Boneberg, M. Tresp, M. Ochmann, H.-J. Münzer, and P. Leiderer. Timeresolved measurements of the response of a STM tip upon illumination with a nanosecond laser pulse. Appl. Phys. A, 66:615-619, 1998. Cited on page 79.

[184] L. Seiffert, F. Süßmann, S. Zherebtsov, P. Rupp, C. Peltz, E. Rühl, M. F. Kling, and T. Fennel. Competition of single and double rescattering in the strong-field photoemission from dielectric nanospheres. Applied Physics B, 122:101, 2016. Cited on page 90.

[185] H. Bradt. Astrophysical processes. Supplement to Chapter 3. Camb. U. Press, 2008. Cited on page 93.

[186] Z. Lin, L. V. Zhigilei, and V. Celli. Electron-phonon coupling and electron heat capacity of metals under conditions of strong electron-phonon nonequilibrium. Phys. Rev. B, 77:075133, 2008. Cited on pages 94 and 95. 
[187] E. Majchrzak and J. Dziatkiewicz. Application of the two-temperature model for a numerical study of multiple laser pulses interactions with thin metal films. Scientific Research of the Institute of Mathematics and Computer Science, 11:6370, 2012. Cited on page 95.

[188] C. Kealhofer, S. M. Foreman, S. Gerlich, and M. A. Kasevich. Ultrafast lasertriggered emission from hafnium carbide tips. Phys. Rev. B, 86:035405, 32012. Cited on page 95.

[189] S.-S. Wellershoff. Untersuchungen zur Energierelaxationsdynamik in Metallen nach Anregung mit ultrakurzen Laserpulsen. PhD thesis. FU Berlin, 2000. Cited on page 95 . 



\section{Author contributions, publications and conference talks}

This cumulative thesis comprises the following two publications:

1. Phase space manipulation of free-electron pulses from metal nanotips using combined terahertz near fields and external biasing

L. Wimmer, O. Karnbach, G. Herink, and C. Ropers.

Physical Review B 95165416 (2017)

2. Clocking plasmon nanofocusing by THz near-field streaking

L. Wimmer, B. Schröder, M. Sivis, G. Herink, and C. Ropers.

Appl. Phys. Lett. 111, 131102 (2017)

These articles present original work of the author (L. Wimmer) regarding the design and implementation of the experiments, the data evaluation and the simulations. The contributions of the co-authors and others are listed in the following.

- The nanotips were fabricated by K. Ahlborn and B. Schröder.

- The measurements presented in (1) were recorded by O. Karnbach in the frame of his bachelor thesis in collaboration with the author.

- The simulations of the streaking spectrograms presented in (1) were developed on the basis of a previous version written by G. Herink (see Ref. [15]).

- The simulations presented in (2) are based on a code written by B. Schröder.

The interpretation of the results was discussed by all authors of the respective publications. The manuscripts of both publications were written by L.Wimmer and C. Ropers, in (1) with contributions from G. Herink. 


\section{Publications}

The following articles originated over the course of this work, and are not a direct part of this cumulative thesis:

- Highly nonlinear and ultrafast optical phenomena in metallic nanostructures

L. Wimmer, M. Sivis, G. Herink, S. V. Yalunin, K. E. Echternkamp, and C. Ropers.

Ultrafast Dynamics Driven by Intense Light Pulses. Springer International Publishing, 2016. 233-258.

- THz-controlled photoelectron emission from nanotips L. Wimmer, G. Herink, K. E. Echternkamp, S. V. Yalunin, D. R. Solli, M. Gulde, and C. Ropers. Ultrafast phenomena XIX - proceedings. Springer International Publishing, 2015. 630-632.

- Field emission at terahertz frequencies: AC-tunneling and ultrafast carrier dynamics G. Herink, L. Wimmer, and C. Ropers.

New J. Phys. 16 (2014).

- Terahertz control of nanotip photoemission

L. Wimmer, G. Herink, D. R. Solli, S. V. Yalunin, K. E. Echternkamp, and C. Ropers.

Nature Physics 10, 432 (2014).

\section{Conference talks}

- Tracking Plasmon Propagation by Terahertz-Streaking at Metal Nanotips,

Frühjahrstagung der DPG (SKM) 2017, Dresden.

- Electron pulse reshaping by $\mathbf{T H z}$ streaking at metal nanotips, IRMMW-THz 2016, Kopenhagen, Danmark. (Finalist for Best Student Paper Award)

- Photoelectron trajectory control in THz near-fields at metal nanotips, EMN Meeting on Terahertz 2016, San Sebastian, Spain. (Invited)

- Tuning of electron dynamics in THz near-field streaking at nanotips, UNO-4 (International Workshop on Ultrafast Nanooptics) 2015, Bad Dürkheim. 
- THz streaking at metal nanotips,

META'15 (International Conference on Metamaterials, Photonic Crystals and Plasmonics) 2015, New York, USA.

- Mapping THz near-fields with nanotip streaking,

CLEO (Conference on Lasers and Electro-optics) Europe 2015, München.

- Probing electron relaxation in single metal nanotips with terahertzinduced field emission,

Frühjahrstagung der DPG (SKM) 2015, Berlin.

- Near-field streaking at nanotips with single-cycle THz transients, NFO 13 (Near-field optics) 2014, Salt Lake City, USA.

- THz-Controlled Photoelectron Emission from Nanotips, Ultrafast Phenomena XIX 2014, Okinawa, Japan.

- Field-driven THz-streaking of Nanotip Photoemission, Frühjahrstagung der DPG (SKM) 2014, Dresden. 



\section{Curriculum Vitae}

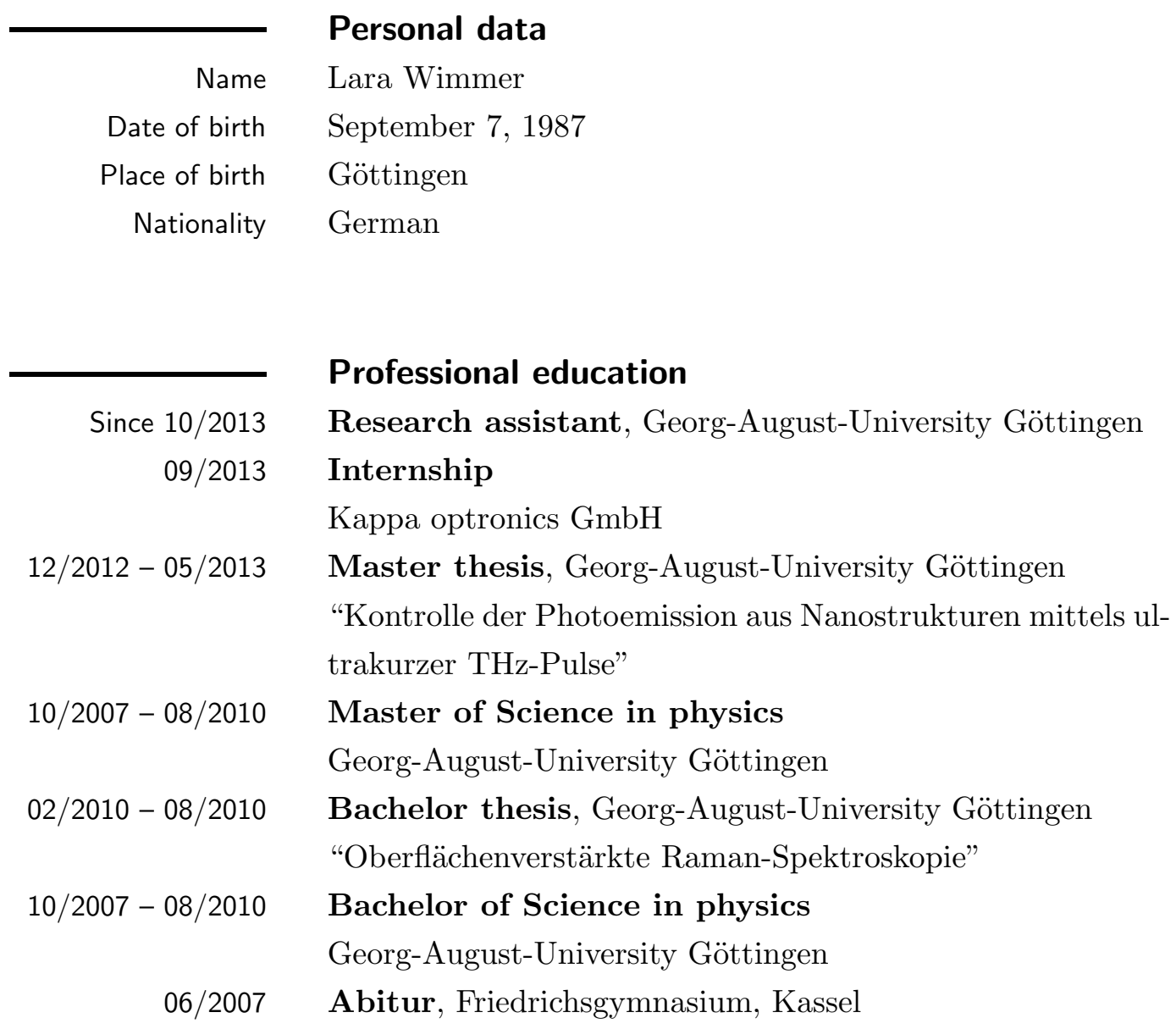





\section{Danksagung}

Selbstverständlich ist auch diese Arbeit nicht ohne Unterstützung entstanden. An dieser Stelle möchte ich mich bei allen bedanken, die mir geholfen haben.

Mein erster Dank gilt meinem Doktorvater Claus Ropers, der diese Arbeit erst ermöglicht hat. Ich danke ihm für die vielen guten Ratschläge und Ideen, für seine geduldige Korrektur zahlreicher Texte, für sein klares, hilfreiches Feedback und für die Möglichkeit zur Teilnahme an internationalen Konferenzen.

Bei meinem Zweitbetreuer Tim Salditt möchte ich mich für die inspirierenden Gespräche bedanken, die mir immer zu neuen Sichtweisen und frischer Motivation verholfen haben. Stefan Mathias danke ich für die freundliche Übernahme des Zweitgutachtens.

Georg Herink hat mich schon während meiner Masterarbeit betreut und stand mir auch danach weiterhin mit Rat und Tat zur Seite. Georg hat ein magisches Händchen für Laser und er gibt sein Wissen gern weiter. Sein Enthusiasmus für die Physik, seine Energie und gute Laune und seine Freundlichkeit machen die Zusammenarbeit mit ihm immer sehr angenehm. Von Georg habe ich gelernt, dass eine gute Präsentation vom Spannungsbogen her immer an eine Geschichte am Lagerfeuer erinnern sollte und dass man manchmal einfach einen Flachwitz braucht.

Der gesamten Arbeitsgruppe danke ich für die außergewöhnlich gute Arbeitsatmosphäre. Dabei möchte ich mich insbesondere bei Katharina Priebe, Armin Feist und Benjamin Schröder bedanken.

Ich danke Katharina für ihre kompetente Hilfsbereitschaft, ihre Freundschaft und natürlich für die regelmäßige Pausenbegleitung inklusive Banane.

Armin danke ich für die vielen guten Gespräche, unter anderem über Elektronenmikroskopie, und die Grillabende im Sommer auf seinem Balkon.

Mit Benjamin habe ich bei der Umsetzung von Streaking an plasmonischen Nanospitzen zusammengearbeitet. Ich danke ihm für das geduldige Beantworten meiner Fragen über Plasmonik und für die Korrektur der entsprechenden Kapitel.

Oliver Karnbach, Thomas Rittmann und Simon Lange habe ich bei ihren Abschlussarbeiten betreut. Es war mir eine Freude, mit ihnen zu arbeiten! In Zusammenarbeit mit Oliver Karnbach sind die Messungen entstanden, die Grundlage für die Veröffentlichung in Kapitel 6 sind.

Karin Ahlborn hat die Spitzen für mein Experiment geätzt, unseren Laser mit frischen Filterkartuschen versorgt und die Wartungsarbeiten für unsere Vakuumtechnik 
übernommen. Für ihre tatkräftige Hilfe und Unterstützung bin ich ihr sehr dankbar. Bei Anna-Lena Robisch möchte ich mich für die vielen guten Gespräche und die sorgfältige Korrektur meiner Arbeit bedanken.

Meinen Eltern Heidrun und Robert Wimmer danke ich für die liebevolle Unterstützung und den Rückhalt während des gesamten Studiums einschließlich der Promotion.

Mein Freund Karthik KrishneGowda hat mich insbesondere in den letzten Monaten vor Abgabe dieser Arbeit nach Kräften unterstützt: Er hat mich besucht, bekocht und zum Lachen gebracht. Danke dir! 